\title{
A B-SPLINE-LIKE BASIS FOR THE POWELL-SABIN 12-SPLIT BASED ON SIMPLEX SPLINES
}

\author{
ELAINE COHEN, TOM LYCHE, AND RICHARD F. RIESENFELD
}

\begin{abstract}
We introduce a simplex spline basis for a space of $C^{1}$-quadratics on the well-known Powell-Sabin 12-split triangular region. Among its many important desirable properties, we show that it has an associated recurrence relation for evaluation and differentiation. Also developed are a Marsden-like identity, quasi-interpolants, approximation methods exhibiting unconditional stability, a subdivision scheme, and smoothness conditions across macro-element edges.
\end{abstract}

\section{Contents}

1. Introduction

1.1. Background

1.2. Notation

1.3. PS12-Split

2. Simplex splines over PS12-split

2.1. Recurrence relations: Basis form

3. A Marsden-like identity

3.1. Proof of the Marsden-like identity

3.2. Linear independence

3.3. Differentiation

4. Evaluation algorithms

5. Subdivision

5.1. Computing the knot insertion matrix

5.2. Coefficient averaging algorithm

6. Stability of the S-basis and the distance to the control points

6.1. Linear and quadratic quasi-interpolant

6.2. Stability of the quadratic S-spline basis

6.3. Distance between a surface and its control points

7. Smooth surfaces joins: Conditions on PS12 refinements of triangulations

8. Approximation methods

8.1. Lagrange interpolation

8.2. Hermite interpolation

8.3. Quasi-interpolants

9. Concluding remarks

Appendix A. Proof of Recurrence Relation Theorem 2.3 1703

Acknowledgements

Received by the editor October 25, 2010 and in revised form, November 3, 2011. 2010 Mathematics Subject Classification. Primary 41A15, 65D07, 65D17, 65D05. 


\section{INTRODUCTION}

Surfaces defined over triangulations have widespread application in many areas ranging from finite element analysis and physics and engineering applications to the entertainment industry. For many of these applications piecewise linear surfaces do not offer sufficient smoothness. To obtain $C^{1}$ smoothness, one must either use quintic polynomials with 21 degrees of freedom over each triangle or use lower degree macroelements that subdivide each triangle into a number of subtriangles. Thus far, the second approach has largely been based on using the Bernstein-Bézier basis on each subtriangle, manually enforcing the smoothness internal to each triangle and solving the resulting constrained system.

In this paper we introduce the S-spline basis, a B-spline like basis, over a single macroelement known as the Powell-Sabin 12-split (PS12-split). Internal to the macroelement, each of the 12 basis elements is $C^{1}$ and the basis is unconditionally stable independent of the shape of the macroelement. Analogous results for analytical and shape properties, so inextricably intertwined in the B-spline/Bézier formulation of surfaces, are shown for the S-spline basis.

1.1. Background. Consider a triangulation $\mathcal{T}_{1}$ of a domain $\Omega \in \mathbb{R}^{2}$ and integers $r, d$ with $-1 \leq r<d$. Let

$$
\mathbb{S}_{d}^{r}\left(\mathcal{T}_{1}\right):=\left\{f \in C^{r}(\Omega): f_{\mid T} \text { is a polynomial of degree } d, \forall T \in \mathcal{T}_{1}\right\}
$$

be the space of piecewise $C^{r}$ polynomials of degree $d$ over $\mathcal{T}_{1}$. To evaluate $f \in$ $\mathbb{S}_{d}^{r}\left(\mathcal{T}_{1}\right)$ the standard approach is to represent $f$ piecewise using a Bernstein-Bézier representation on each triangle in $\mathcal{T}_{1}$ and use the de Casteljau Recursive Algorithm [13. for evaluation. It was shown in [15] that the $L_{q}$ normalized Bernstein-Bézier basis on a triangle is stable in any $L_{q}$ norm.

Now suppose $\mathcal{T}_{1}$ is obtained from a coarser triangulation $\mathcal{T}_{0}$ by splitting each triangle in $\mathcal{T}_{0}$ into subtriangles. Suppose for each triangle $\Delta \in \mathcal{T}_{0}$ there exists a basis $\mathcal{B}_{\Delta}$ of the space $\mathbb{S}_{d}^{r}\left(\mathcal{T}_{1}\right)$ restricted to $\Delta$ that has:

- stable recurrence relations

- differentiation formula

- minimal support

- local linear independence

- nonnegative partition of unity

- explicit dual functionals

- $L_{q}$ stable basis, $1 \leq q \leq \infty$

- simple conditions for $C^{1}$ joins to neighboring triangles

- well conditioned collocation matrices for Lagrange and Hermite interpolation using certain sites, and

- subdivision algorithms of Lane-Riesenfeld type.

These characteristics yield a B-spline-like basis within each $\Delta$, but they do not give a $\mathrm{B}$-spline basis for the whole triangulation $\mathcal{T}_{1}$. So $\mathcal{B}$ behaves like the triangular Bernstein-Bézier basis across the edges of $\mathcal{T}_{0}$, but like a B-spline internal to each triangle of $\mathcal{T}_{0}$. The characteristics of this basis make it unnecessary to convert it to a Bernstein-Bézier representation on each of the subtriangles of $\Delta$. Instead there is a single control mesh to facilitate control and early visualization of the surface over each triangle $\Delta$ in $\mathcal{T}_{0}$. 

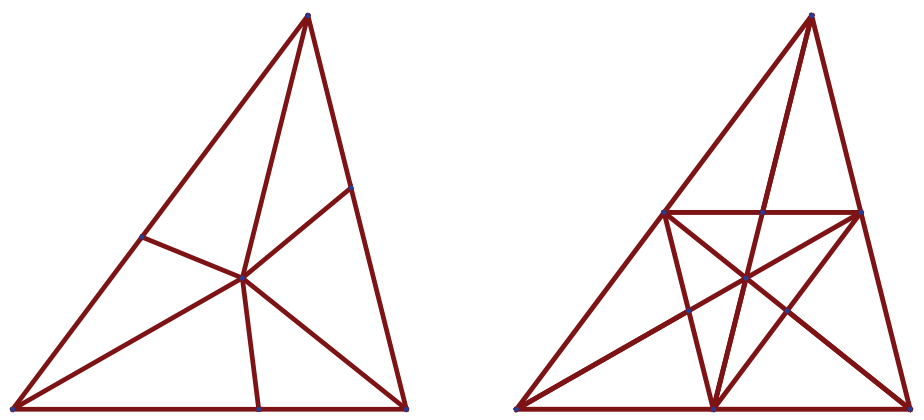

FiguRE 1. Left: PS6-split, and right: PS12-split configurations.

We develop a quadratic $C^{1}$ spline basis with the above desired behavior on each element of $\mathcal{T}_{0}$ that has been split according to the scheme of the Powell-Sabin 12Split (PS12) 20]. Each triangle in $\mathcal{T}_{0}$ is split into 12 subtriangles delineated by the complete graph connecting all vertices and edge midpoints. (See Fig. 1). The union of the bases over $\mathcal{T}_{0}$ can be used to represent the space of $C^{1}$ quadratic splines on $\mathcal{T}_{1}$.

An interpolatory subdivision scheme for the PS12-split introduced in [9] can be used to evaluate a quadratic PS12 specified surface on an arbitrary triangulation.

There are other notable approaches to constructing spline spaces over triangulations, in particular:

- The nodal basis, dual to the degrees of freedom for the Hermite constraints, is commonly employed [3] in finite element calculations.

- Elaborate constructions exist based on perturbing the knots of a BernsteinBézier triangle representation in 6, 7, 12, 22, However, the space thus obtained, represented by a simplex spline basis over the perturbed knot set, depends on the particular perturbations applied. Also, evaluation based on simplex spline recursion is slow [10, 11.

- An approach employing Delaunay configurations based on points in general position is introduced and studied by Neamtu in [18, 19]. This approach uses a sum of simplex splines as basis functions and generalizes many Bspline properties; however, there is no associated recurrence relation relating these functions to basis functions of lower degree.

- Box splines [2] can be applied to uniform triangulations.

- Schemes using a different split rule, called the Powell-Sabin 6-Split (PS6) [20], create $\mathcal{T}_{1}$ from $\mathcal{T}_{0}$ by dividing each triangle of $\mathcal{T}_{0}$ into 6 subtriangles by connecting the incenter of a triangle to its vertices and to the incenters of its adjacent triangles. Dierckx [8] introduced a B-spline-like basis for $C^{1}$ quadratics on a $\mathcal{T}_{1}$ split of $\mathcal{T}_{0}$ according to the PS6 rules. Since there is no recurrence relation for this basis, evaluation is done by transforming them to the Bernstein-Bézier representation on each of the 6 subtriangles. Being nonnegative and forming a partition of unity, the basis functions can be manipulated with their corresponding control points. They form a stable 
basis in the $L_{q}$ norm, $1 \leq q \leq \infty$, where the stability constant depends on the smallest angle in the underlying triangulation [16, 23.

- The Dierckx basis was recently extended in 25] to a space of $C^{2}$ quintics on triangulations amenable to the PS6-split.

In this paper we introduce the S-spline basis, a quadratic simplex spline basis $\mathcal{S}_{2}$, for the PS12 macroelement. In addition to having simplex spline properties, we prove that the S-spline basis has many desirable B-spline properties. In particular, they form a partition of unity, provide a recurrence relation down to hat functions, satisfy a Marsden-like identity, and exhibit $L_{q}$ stability for a scaled version. Furthermore, the restriction of each basis element to the boundary edges of the macroelement reduces to a standard univariate B-spline. A control mesh can be formed that mimics the shape of the surface and exhibits distance $O\left(h^{2}\right)$ to any one of its control points from its surface, where $h$ is the length of the longest edge. We obtain a pyramidal evaluation algorithm in terms of the control points that is strikingly reminiscent of the analogous one for triangular Bézier surfaces. An unusual hybrid, the control mesh presents both triangular and quadrilateral connectivity.

The S-spline basis can be used to represent surfaces over arbitrary triangulations. We derive conditions for $C^{1}$ smoothness across macro-triangle edges in terms of control points similar to the triangular Bézier case.

We obtain two algorithms for the subdivision approach considered. One subdivides each macroelement into 4 submacro-elements so that the S-spline basis elements are subdivided giving formulas analogous to Oslo Algorithm 1 [4]. The other is an analog of the the Lane-Riesenfeld Algorithm for Bézier surfaces [14. Repeated subdivision converges quadratically due to the aforementioned distance result and can be used for evaluation, rendering, and other computations commonly associated with freeform geometric modeling.

Now we preface the ensuing mathematical development with a description of the notation consistently used throughout.

1.2. Notation. We use small boldface letters to denote vectors and capital boldface letters for matrices, while calligraphic fonts like $\mathcal{R}, \mathcal{S}$ indicate sets. With function spaces we associate symbols like $\mathbb{S}$.

The symbol $\mathbb{R}^{m, n}$ denotes the class of $m \times n$ real matrices $\boldsymbol{A}, \boldsymbol{R}, \ldots$ We denote the unit vectors in $\mathbb{R}^{m}$ by $\boldsymbol{e}_{1}, \ldots, \boldsymbol{e}_{m}$, the identity matrix with $\boldsymbol{I}$, and $\boldsymbol{e}:=$ $[1,1, \ldots, 1]^{T} \in \mathbb{R}^{m}$. We write $\# \boldsymbol{A}$ for the number of columns of $\boldsymbol{A}$, and $\# \mathcal{S}$ for the cardinality of set $\mathcal{S}$. If $\boldsymbol{A} \in \mathbb{R}^{m, n}$ and $\boldsymbol{i}=\left[i_{1}, \ldots, i_{r}\right]^{T}, \boldsymbol{j}=\left[j_{1}, \ldots, j_{s}\right]^{T}$ with $1 \leq i_{1}<\cdots<i_{r} \leq m, 1 \leq j_{1}<\cdots<j_{s} \leq n$, then $\boldsymbol{A}(\boldsymbol{i}, \boldsymbol{j}) \in \mathbb{R}^{r, s}$ denotes the matrix whose $k, \ell$ element is $a_{i_{k}, j_{\ell}}$. In particular, $\boldsymbol{c}(\boldsymbol{i})$ denotes the vector whose $j$ th element is $c_{i_{j}}$. For a vector of functions $\boldsymbol{f}$ the symbol $\boldsymbol{f}(\boldsymbol{i})$ denotes the subvector of functions whose $j$ th element is $f_{i_{j}}$. The support of a function $f$, denoted by $\operatorname{supp}(f)$, is the closure of the set of values in the domain of $f$ at which $f$ is nonzero.

We denote by $\Pi_{d}\left(\mathbb{R}^{2}\right)$ the space of bivariate polynomials with real coefficients of total degree $\leq d$, i.e., the span of all monomials of the form $x_{1}^{i_{1}} x_{2}^{i_{2}}$, where the nonnegative integers satisfy $i_{1}+i_{2} \leq d$. The dimension of $\Pi_{d}\left(\mathbb{R}^{2}\right)$ is $\nu_{d}:=$ $(d+1)(d+2) / 2$. $\mathbb{S}_{d}^{r}(\Delta)$ denotes the space of piecewise polynomials of degree $d$ and smoothness $C^{r}$ on a triangulation $\Delta$ of $\mathbb{R}^{2}$. 


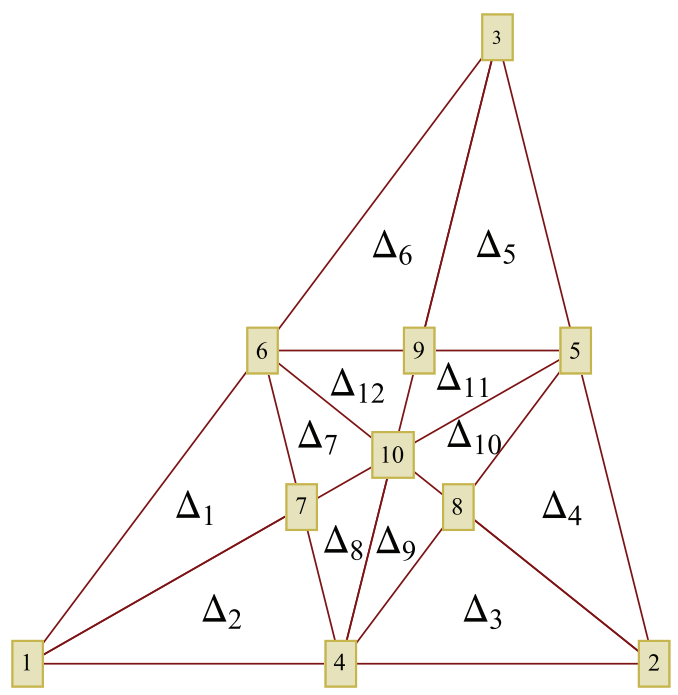

Figure 2. Reference labels for vertices and induced 12 subtriangles for the PS12-split.

To denote the closed or open convex hull of a set $\mathcal{S}$ we use $\operatorname{ch}(\mathcal{S})$ or $\operatorname{ch}(\mathcal{S})^{o}$, respectively. When $\mathcal{S}$, consisting of the columns of $\boldsymbol{A}$, spans $\mathbb{R}^{m}$, we say simply "A spans $\mathbb{R}^{m} "$.

1.3. PS12-Split. Prominent among the known macroelements is the Powell-Sabin 12-split (PS12-split). Given three noncollinear points $\boldsymbol{p}_{1}, \boldsymbol{p}_{2}, \boldsymbol{p}_{3}$ in $\mathbb{R}^{2}$, the triangle

$$
\Delta:=\operatorname{ch}\left(\left\{\boldsymbol{p}_{1}, \boldsymbol{p}_{2}, \boldsymbol{p}_{3}\right\}\right)
$$

with vertices $\boldsymbol{p}_{1}, \boldsymbol{p}_{2}, \boldsymbol{p}_{3}$ will serve as our macrotriangle. The PS12-split divides $\Delta$ into 12 subtriangles delineated by the complete graph formed by the 3 original triangle vertices and its 3 edge midpoints (see Fig. 2).

We number the vertices $\boldsymbol{p}_{1}, \ldots, \boldsymbol{p}_{10}$ and the triangles $\Delta_{1}, \ldots, \Delta_{12}$ of the PS12split as depicted in Figure 2, Note that

$$
\begin{aligned}
\boldsymbol{p}_{4}:=\frac{1}{2}\left(\boldsymbol{p}_{1}+\boldsymbol{p}_{2}\right), \quad \boldsymbol{p}_{5}:=\frac{1}{2}\left(\boldsymbol{p}_{2}+\boldsymbol{p}_{3}\right), \quad \boldsymbol{p}_{6}:=\frac{1}{2}\left(\boldsymbol{p}_{1}+\boldsymbol{p}_{3}\right), \\
\boldsymbol{p}_{7}:=\frac{1}{2}\left(\boldsymbol{p}_{4}+\boldsymbol{p}_{6}\right), \quad \boldsymbol{p}_{8}:=\frac{1}{2}\left(\boldsymbol{p}_{4}+\boldsymbol{p}_{5}\right), \quad \boldsymbol{p}_{9}:=\frac{1}{2}\left(\boldsymbol{p}_{5}+\boldsymbol{p}_{6}\right), \\
\boldsymbol{p}_{10}:=\frac{1}{3}\left(\boldsymbol{p}_{1}+\boldsymbol{p}_{2}+\boldsymbol{p}_{3}\right) .
\end{aligned}
$$

$\Delta_{P S 12}$ is the PS12 triangulation of $\Delta$. We follow the convention in [22, to decide in which subtriangle each edge and vertex belongs. The author defines a point $\boldsymbol{p}$ to be in the half-open convex hull of $\Delta_{k}$ if and only if there is a vector $\boldsymbol{\eta}$ with positive slope, and a scalar $\varepsilon>0$ such that $\left\{\boldsymbol{p}+s \boldsymbol{e}_{1}+t \boldsymbol{\eta}: 0<s, t<s+t<\varepsilon\right\}$ is completely contained in the interior of $\Delta_{k}$. The resulting configuration is shown in Figure 3 for a typical triangle. Note that in what subtriangle each edge and vertex is contained depends on the orientation of the triangle. This convention extends to 


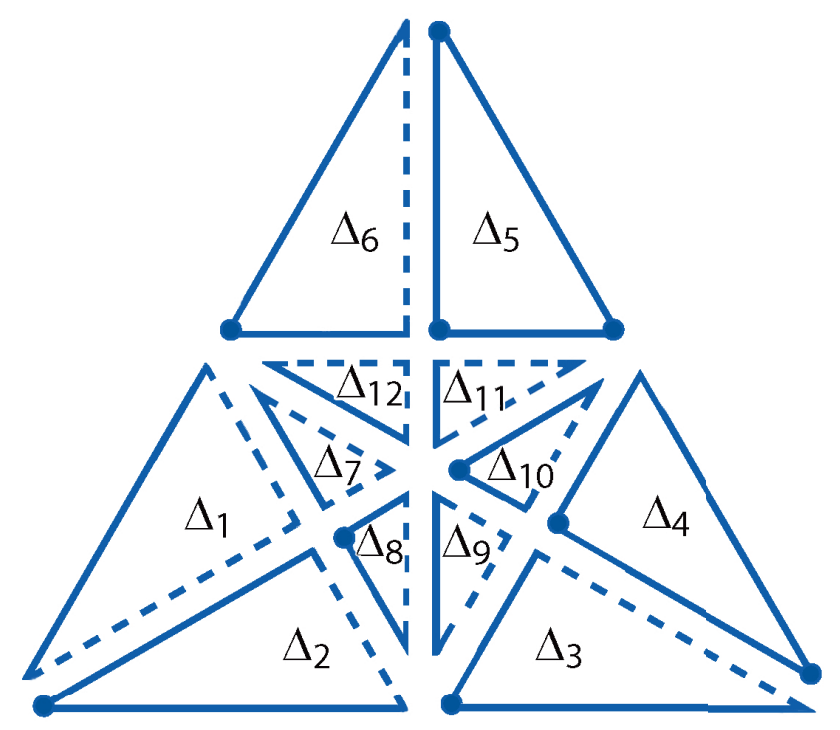

Figure 3. Scheme that uniquely assigns each $\boldsymbol{x} \in \Delta$ to a unique (possibly half-open) subtriangle $\Delta_{k}$.

associate every edge and vertex in the PS12-refinement of an arbitrary triangulation with a unique subtriangle. Thus this uniquely and completely defines a piecewise polynomial at all points of the PS12-refinement of an arbitrary triangulation.

The following algorithm determines a unique subtriangle for a point in $\mathbb{R}^{2}$ with respect to a single PS12 macroelement. The algorithm is consistent with the convention described above for the triangle in Figure 3

Algorithm 1.1. Let $\boldsymbol{x} \in \mathbb{R}^{2}$ and $\Delta$ be partitioned by $\left\{\Delta_{1}, \ldots, \Delta_{12}\right\}$ as in Figure 3 , Then:

(1) Find the barycentric coordinate triple $\left(\beta_{1}, \beta_{2}, \beta_{3}\right)$ of $\boldsymbol{x}$ with respect to $\Delta$.

(2) Compute sw $:=\left[2^{5}, 2^{4}, 2^{3}, 2^{2}, 2^{1}, 2^{0}\right] \boldsymbol{t}$, where $\boldsymbol{t}:=\left[\left(\beta_{1}>\frac{1}{2}\right),\left(\beta_{2} \geq \frac{1}{2}\right),\left(\beta_{3} \geq \frac{1}{2}\right),\left(\beta_{1}>\beta_{2}\right),\left(\beta_{1}>\beta_{3}\right),\left(\beta_{2} \geq \beta_{3}\right)\right]^{T}$.

(3) Select $k$ from the table below.

\begin{tabular}{|c|c|c|c|c|c|c|c|c|c|c|c|c|c|c|}
\hline$s w$ & 38 & 46 & 39 & 19 & 17 & 8 & 25 & 12 & 6 & 7 & 3 & 1 & 0 & 4 \\
\hline$k$ & 1 & 1 & 2 & 3 & 4 & 5 & 5 & 6 & 7 & 8 & 9 & 10 & 11 & 12 \\
\hline
\end{tabular}

(4) $\boldsymbol{x} \in \Delta_{k} . \boldsymbol{x} \notin \Delta$ for other values of sw.

Note that $\boldsymbol{t}$ is a Boolean vector consisting of zeros and ones. For example the second to last component is one if $\boldsymbol{x}$ is closer to $\boldsymbol{p}_{1}$ than to $\boldsymbol{p}_{3}$, constraining it to lie in only one of the triangles $\Delta_{k}, k=1,2,3,7,8,9$.

\section{SimpleX SPLINES OVER PS12-SPLiT}

On the triangulation $\Delta_{P S 12}$ of the triangle $\Delta$, we consider the spline spaces:

$$
\mathbb{S}_{d}:=\mathbb{S}_{d}^{d-1}\left(\Delta_{P S 12}\right), \quad d=0,1,2 .
$$



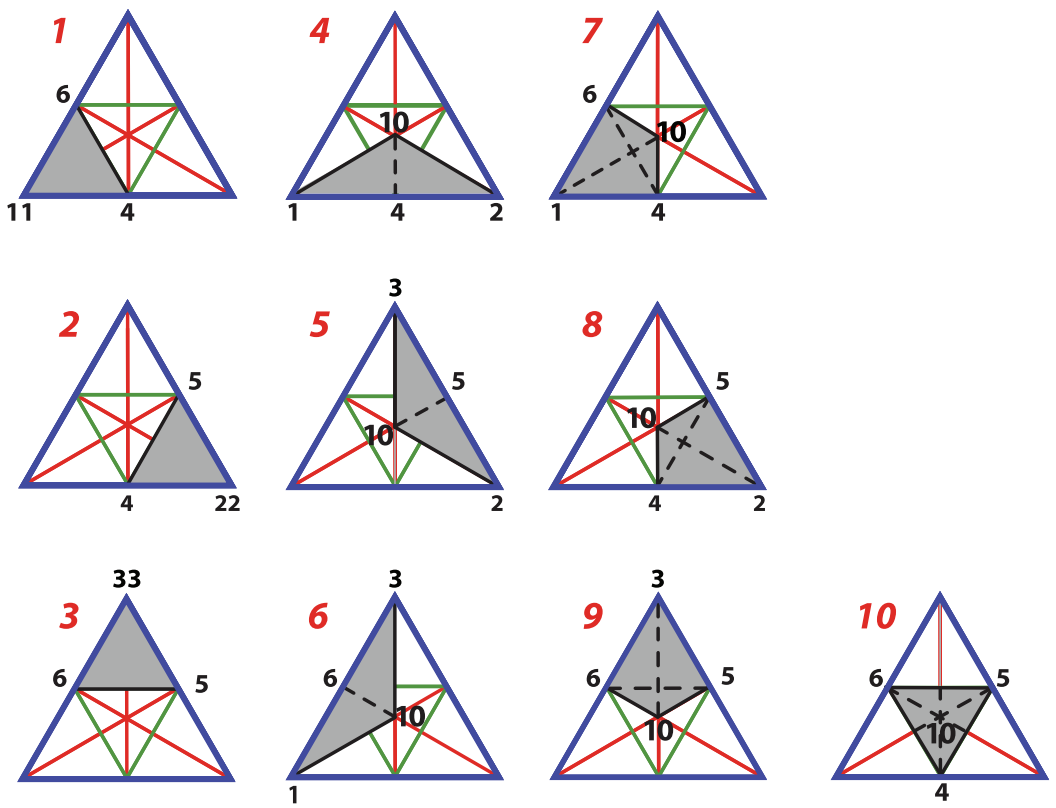

FiguRE 4. Shaded areas indicated support for knot configurations of the 10 linear simplex splines $S_{j, 1}$ on PS12-split.

Since there are 12 triangles and 10 vertices in $\Delta_{P S 12}$ (see Fig. 2), $n_{0}:=\operatorname{dim}\left(\mathbb{S}_{0}\right)=$ 12 and $n_{1}:=\operatorname{dim}\left(\mathbb{S}_{1}\right)=10$, respectively. Similarly, $n_{2}:=\operatorname{dim}\left(\mathbb{S}_{2}\right)=12$, and there is a unique $f \in \mathbb{S}_{2}$ that interpolates function values and gradients at the vertices $\boldsymbol{p}_{1}, \boldsymbol{p}_{2}, \boldsymbol{p}_{3}$ and cross derivatives at the midpoints $\boldsymbol{p}_{4}, \boldsymbol{p}_{5}, \boldsymbol{p}_{6}$ [20].

Definition 2.1. For $d=0,1,2$, we define S-splines for $\mathbb{S}_{d}$ by

$$
S_{j, d}=\frac{v_{j, d}}{\nu_{d}} M\left(\cdot \mid \boldsymbol{K}_{j, d}\right), \quad j=1, \ldots, n_{d}
$$

where $M\left(\cdot \mid \boldsymbol{K}_{j, d}\right)$ is a degree $d$ simplex spline normalized to have unit integral, and $v_{j, d}:=v\left(\Omega_{j, d}\right)$ is the 2-dimensional volume (area) of $\Omega_{j, d}:=\operatorname{supp}\left(S_{j, d}\right)=\operatorname{ch}\left(\boldsymbol{K}_{j, d}\right)$. The set

$$
\mathcal{S}_{d}:=\left\{S_{1, d}, \ldots, S_{n_{d}, d}\right\}, \quad d=0,1,2
$$

is the S-spline basis of degree $d$ for the PS12-split. The knot sets $\boldsymbol{K}_{j, d}$ are defined as follows: $\boldsymbol{K}_{j, 0}$ consists of the three vertices of $\Delta_{j}$, while $\boldsymbol{K}_{j, 1}$ and $\boldsymbol{K}_{j, 2}$ are defined as follows:

$$
\begin{array}{lll}
\boldsymbol{K}_{1,1}=\left[\boldsymbol{p}_{1}^{(2)}, \boldsymbol{p}_{4}, \boldsymbol{p}_{6}\right] & \boldsymbol{K}_{2,1}=\left[\boldsymbol{p}_{2}^{(2)}, \boldsymbol{p}_{5}, \boldsymbol{p}_{4}\right] & \boldsymbol{K}_{3,1}=\left[\boldsymbol{p}_{3}^{(2)}, \boldsymbol{p}_{6}, \boldsymbol{p}_{5}\right] \\
\boldsymbol{K}_{4,1}=\left[\boldsymbol{p}_{1}, \boldsymbol{p}_{4}, \boldsymbol{p}_{2}, \boldsymbol{p}_{10}\right] & \boldsymbol{K}_{5,1}=\left[\boldsymbol{p}_{2}, \boldsymbol{p}_{5}, \boldsymbol{p}_{3}, \boldsymbol{p}_{10}\right] & \boldsymbol{K}_{6,1}=\left[\boldsymbol{p}_{3}, \boldsymbol{p}_{6}, \boldsymbol{p}_{1}, \boldsymbol{p}_{10}\right] \\
\boldsymbol{K}_{7,1}=\left[\boldsymbol{p}_{1}, \boldsymbol{p}_{4}, \boldsymbol{p}_{10}, \boldsymbol{p}_{6}\right] & \boldsymbol{K}_{8,1}=\left[\boldsymbol{p}_{2}, \boldsymbol{p}_{5}, \boldsymbol{p}_{10}, \boldsymbol{p}_{4}\right] & \boldsymbol{K}_{9,1}=\left[\boldsymbol{p}_{3}, \boldsymbol{p}_{6}, \boldsymbol{p}_{10}, \boldsymbol{p}_{5}\right] \\
\boldsymbol{K}_{10,1}=\left[\boldsymbol{p}_{4}, \boldsymbol{p}_{5}, \boldsymbol{p}_{6}, \boldsymbol{p}_{10}\right], & &
\end{array}
$$



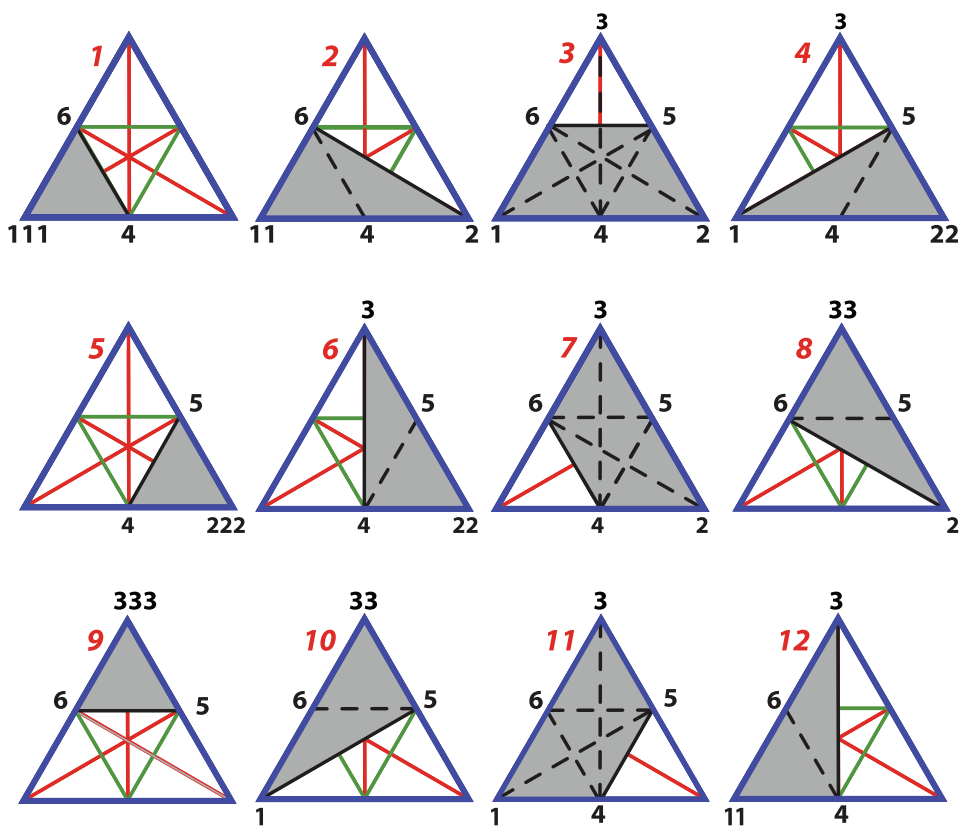

FiguRE 5. Shaded areas indicated support for knot configurations of the 12 quadratic simplex splines $S_{j, 2}$ on PS12-split.

$$
\begin{array}{lll}
\boldsymbol{K}_{1,2}=\left[\boldsymbol{p}_{1}^{(3)}, \boldsymbol{p}_{4}, \boldsymbol{p}_{6}\right] & \boldsymbol{K}_{2,2}=\left[\boldsymbol{p}_{1}^{(2)}, \boldsymbol{p}_{4}, \boldsymbol{p}_{2}, \boldsymbol{p}_{6}\right] & \boldsymbol{K}_{3,2}=\left[\boldsymbol{p}_{1}, \boldsymbol{p}_{4}, \boldsymbol{p}_{2}, \boldsymbol{p}_{5}, \boldsymbol{p}_{6}\right] \\
\boldsymbol{K}_{4,2}=\left[\boldsymbol{p}_{2}^{(2)}, \boldsymbol{p}_{5}, \boldsymbol{p}_{1}, \boldsymbol{p}_{4}\right] & \boldsymbol{K}_{5,2}=\left[\boldsymbol{p}_{2}^{(3)}, \boldsymbol{p}_{5}, \boldsymbol{p}_{4}\right] & \boldsymbol{K}_{6,2}=\left[\boldsymbol{p}_{2}^{(2)}, \boldsymbol{p}_{5}, \boldsymbol{p}_{3}, \boldsymbol{p}_{4}\right] \\
\boldsymbol{K}_{7,2}=\left[\boldsymbol{p}_{2}, \boldsymbol{p}_{5}, \boldsymbol{p}_{3}, \boldsymbol{p}_{6}, \boldsymbol{p}_{4}\right] & \boldsymbol{K}_{8,2}=\left[\boldsymbol{p}_{3}^{(2)}, \boldsymbol{p}_{6}, \boldsymbol{p}_{2}, \boldsymbol{p}_{5}\right] & \boldsymbol{K}_{9,2}=\left[\boldsymbol{p}_{3}^{(3)}, \boldsymbol{p}_{6}, \boldsymbol{p}_{5}\right] \\
\boldsymbol{K}_{10,2}=\left[\boldsymbol{p}_{3}^{(2)} \boldsymbol{p}_{6}, \boldsymbol{p}_{1}, \boldsymbol{p}_{5}\right] & \boldsymbol{K}_{11,2}=\left[\boldsymbol{p}_{3}, \boldsymbol{p}_{6}, \boldsymbol{p}_{1}, \boldsymbol{p}_{4}, \boldsymbol{p}_{5}\right] & \boldsymbol{K}_{12,2}=\left[\boldsymbol{p}_{1}^{(2)}, \boldsymbol{p}_{4}, \boldsymbol{p}_{3}, \boldsymbol{p}_{6}\right]
\end{array}
$$

Note that superscript indicates multiplicity so that $\left[\boldsymbol{p}_{1}^{(2)}, \boldsymbol{p}_{4}, \boldsymbol{p}_{6}\right]=\left[\boldsymbol{p}_{1}, \boldsymbol{p}_{1}, \boldsymbol{p}_{4}, \boldsymbol{p}_{6}\right]$. Comprised of points in $\mathbb{R}^{2}$, the $d+3$ columns of $\boldsymbol{K}_{j, d}$ are called the knots of $S_{j, d}$. A spline of degree $d$ on $\Delta_{P S 12}$ is any linear combination $\sum_{j=1}^{n_{d}} c_{j} S_{j, d}$.

The supports of the 10 linear S-splines are shown in Figure 4. There are 4 distinctly structured types grouped as follows: the 3 corners $S_{1,1}, S_{2,1}, S_{3,1}$, the 3 midpoint edges $S_{4,1}, S_{5,1}, S_{6,1}$, the 3 Egyptian pyramids $S_{7,1}, S_{8,1}, S_{9,1}$, and the center pyramid $S_{10,1}$. For quadratic S-splines shown in Figure 5 there are three distinct types, namely, the 3 corners $S_{1,2}, S_{5,2}, S_{9,2}$, the 6 edges $S_{2,2}, S_{4,2}, S_{6,2}, S_{8,2}, S_{10,2}$, $S_{12,2}$ and the 3 with trapezoidal support $S_{3,2}, S_{7,2}, S_{11,2}$. Observe that all support sets are shaped either like a triangle or a quadrilateral.

The S-splines have the following properties.

Theorem 2.2. For $d=0,1,2, j=1, \ldots, n_{d}$, and $\boldsymbol{x} \in \Delta$ :

(1) Smooth piecewise polynomial: $S_{j, d} \in \mathbb{S}_{d}$.

(2) Nonnegativity and positivity: $S_{j, d}(\boldsymbol{x}) \geq 0, S_{j, d}(\boldsymbol{x})>0$ for $\boldsymbol{x} \in$ $\operatorname{ch}\left(\boldsymbol{K}_{j, d}\right)^{o}$.

(3) Partition of unity: $\sum_{j=1}^{n_{d}} S_{j, d}(\boldsymbol{x})=1$. 
(4) Convex hull: If the S-spline coefficients $\boldsymbol{c}_{j}$ are points in $\mathbb{R}^{2}$ or $\mathbb{R}^{3}$, then $f(\boldsymbol{x}):=\sum_{j=1}^{n_{d}} \boldsymbol{c}_{j} S_{j, d}(\boldsymbol{x})$ is contained in $\operatorname{ch}\left(\left\{\boldsymbol{c}_{j}\right\}_{1}^{n_{d}}\right)$.

(5) Local linear independence: On each of the 12 subtriangles $\Delta_{k}$ the nonzero $S_{j, d}$ constitute a basis for $\Pi_{d}\left(\mathbb{R}^{2}\right)$.

(6) Basis: The $S$-basis $\mathcal{S}_{d}:=\left\{S_{1, d}, \ldots, S_{n_{d}, d}\right\}$ is a basis for $\mathbb{S}_{d}$.

(7) Degree 0: $S_{j, 0}=\chi_{\Delta_{j}}$, the characteristic function of $\Delta_{j}$.

(8) Linear hat: $S_{j, 1}$ is a piecewise linear hat function with,

$$
S_{j, 1}\left(\boldsymbol{p}_{i}\right)=\delta_{i, j}, \quad i, j=1, \ldots, 10,
$$

(9) Edge restriction: For $d=1,2$ a spline $f=\sum_{j=1}^{n_{d}} c_{j} S_{j, d}$ reduces to a univariate spline with one interior (midpoint) knot along an edge of $\Delta$. In particular, for $d=1$ along the edge $\operatorname{ch}\left(\left\{\boldsymbol{p}_{1}, \boldsymbol{p}_{2}\right\}\right)$,

$$
f\left((1-t) \boldsymbol{p}_{1}+t \boldsymbol{p}_{2}\right)=c_{1} B_{1,1}(t)+c_{4} B_{2,1}(t)+c_{2} B_{3,1}(t), t \in[0,1]
$$

where $\left\{B_{j, 1}\right\}_{j=1}^{3}$ is the set of 3 consecutive univariate linear $B$-splines on the knot vector $\left[0,0, \frac{1}{2}, 1,1\right]$, normalized to form a partition of unity on $[0,1]$. Similarly, for $d=2$,

$$
f\left((1-t) \boldsymbol{p}_{1}+t \boldsymbol{p}_{2}\right)=c_{1} B_{1,2}(t)+c_{2} B_{2,2}(t)+c_{4} B_{3,2}(t)+c_{5} B_{4,2}(t), t \in[0,1],
$$

where $\left\{B_{j, 2}\right\}_{j=1}^{4}$ is the set of 4 consecutive univariate quadratic B-splines on the knot vector $[0,0,0,1 / 2,1,1,1]$. Again, they are normalized to form a partition of unity.

Proof. As bona fide simplex splines, the elements of the S-basis exhibit the following desirable properties [5, 17]:

(1) $S_{j, d}$ has $d+3$ knots and is a piecewise polynomial of degree $d$.

(2) The support of $S_{j, d}$ is $\operatorname{ch}\left(\boldsymbol{K}_{j, d}\right)$.

(3) $S_{j, d}$ is positive in the interior of its support.

(4) The knot lines of $S_{j, d}$ form the complete graph of the knots.

(5) $S_{j, d}$ has $d+1-m$ continuous derivatives across a knot line containing $m$ collinear knots.

Since all knot lines of $S_{j, d}$ are segments of edges in $\Delta_{P S 12}$ it follows from Property 1 that $S_{j, d}$ is a piecewise polynomial of degree $d$ on $\Delta_{P S 12}$. Moreover, since there are exactly two knots on those knot lines of $S_{j, d}$ containing a point in the interior of $\Delta, S_{j, d} \in C^{d-1}\left(\Delta_{P S 12}\right)$ by Property [5. It follows that $S_{j, d} \in \mathbb{S}_{d}$. There are precisely $\nu_{d}$ nonzero $S_{j, d}$ on each triangle $\Delta_{k}$ (cf. Figures 4 and 5 ). In particular, it follows from the support, continuity and partition of unity that for $d=0, S_{j, 0}$ is the characteristic function of $\Delta_{j}$, and that for $d=1$ equation (2.6) holds. We show a Marsden-type identity in Section 3 that implies that functions in $\mathbb{S}_{d}$ span $\Pi_{d}\left(\mathbb{R}^{2}\right)$ on every $\Delta_{k}$ and this identity also implies partition of unity, local linear independence, and basis properties. The convex hull property follows from nonnegativity and partition of unity. The edge restriction property will be proved using the recurrence relations in Theorem 2.3 . 

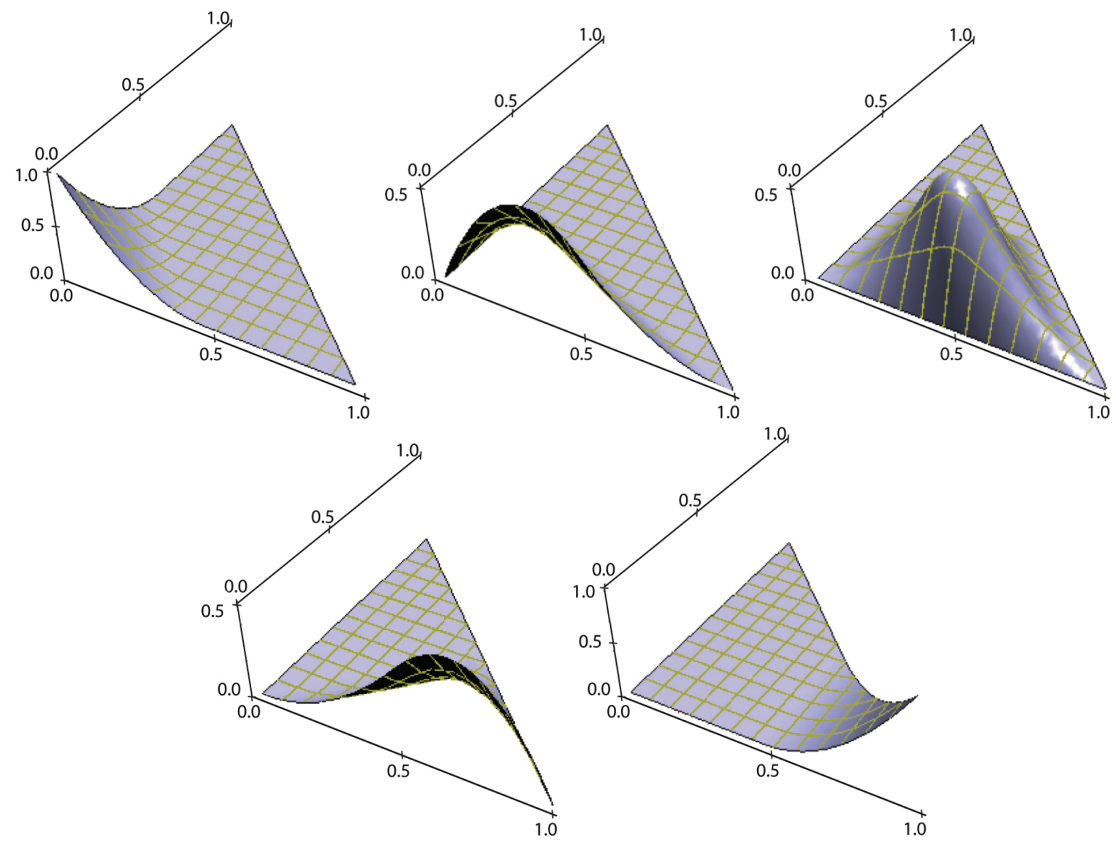

Figure 6 . The S-splines $S_{j, 2}$ for $j=1,2,3,4,5$, on the unit triangle $\boldsymbol{p}_{1}=(0,0), \boldsymbol{p}_{2}=(1,0)$, and $\boldsymbol{p}_{3}=(0,1)$.

Figure6illustrates the edge restriction property along the front edge $\operatorname{ch}\left(\left\{\boldsymbol{p}_{1}, \boldsymbol{p}_{2}\right\}\right)$, where $S_{j, 2}$, for $j=1,2,4,5$, exhibit the proper shapes of a univariate quadratic Bspline. Along the boundary of the triangle, the S-spline $S_{3,2}$, whose support is trapezoidal, has function value 0 , but its cross-boundary derivative is nonzero.

2.1. Recurrence relations: Basis form. Consider the recurrence relation for univariate B-splines $B_{j, d}$ of degree $d$ and with $d+2$ knots $t_{j}, \ldots, t_{j+d+1}$,

$B_{j, d}=\mu_{j, d} B_{j, d-1}+\lambda_{j+1, d} B_{j+1, d-1}, \quad \mu_{j, d}(x):=\frac{x-t_{j}}{t_{j+d}-t_{j}}, \quad \lambda_{j, d}(x):=\frac{t_{j+d}-x}{t_{j+d}-t_{j}}$,

and where $\lambda_{j, d}=\mu_{j, d}:=0$ if $t_{j+d}=t_{j}$. On a knot vector $\boldsymbol{t}:=\left(t_{j}\right)_{j=1}^{m}$ with $t_{1} \leq t_{2} \leq \cdots \leq t_{m}$, the recurrence relation for the $(r:=m-d-1)$ B-splines of degree $d$ that are defined on $\boldsymbol{t}$ can be written in matrix form as,

$$
\left[B_{1, d}, \ldots, B_{r, d}\right]=\left[B_{1, d-1}, \ldots, B_{r+1, d-1}\right] \boldsymbol{R}_{d, \boldsymbol{t}}, \quad t_{1} \leq x<t_{m}
$$

where

$$
\boldsymbol{R}_{d, \boldsymbol{t}}=\left[\begin{array}{cccc}
\mu_{1, d} & & & \\
\lambda_{2, d} & \mu_{2, d} & & \\
& \lambda_{3, d} & \ddots & \\
& & \ddots & \mu_{r, d} \\
& & & \lambda_{r+1, d}
\end{array}\right] \in \mathbb{R}^{r+1, r}
$$


If $t_{k} \leq x<t_{k+1}$ for some integer $k$, then only $B_{k-d, d}(x), \ldots, B_{k, d}(x)$ can be nonzero and (2.9) reduces to the polynomial version

$$
\left[B_{k-d, d}^{k}, \ldots, B_{k, d}^{k}\right]=\left[B_{k-d+1, d-1}^{k}, \ldots, B_{k, d-1}^{k}\right] \boldsymbol{R}_{d, \boldsymbol{t}}^{k},
$$

where $B_{j, d}^{k}$ is the polynomial of degree $\leq d$ representing $B_{j, d}$ on $\left[t_{k}, t_{k+1}\right)$ and

$$
\boldsymbol{R}_{d, \boldsymbol{t}}^{k}:=\left[\begin{array}{cccc}
\lambda_{k-d+1, d} & \mu_{k-d+1, d} & & \\
\ddots & \ddots & & \\
& \ddots & \ddots & \\
& & \lambda_{k, d} & \mu_{k, d}
\end{array}\right] \in \mathbb{R}^{d, d+1} .
$$

We now give analogs of equations (2.9) and (2.11) for the S-spline basis. Consider first (2.9).

Theorem 2.3. If

$$
\boldsymbol{s}_{d}^{T}:=\left[S_{1, d}, \ldots, S_{n_{d}, d}\right], \quad d=0,1,2
$$

then

$$
\boldsymbol{s}_{d}^{T}=\boldsymbol{s}_{d-1}^{T} \boldsymbol{R}_{d}, \quad d=1,2,
$$

where $\boldsymbol{R}_{1} \in \mathbb{R}^{12,10}$ and $\boldsymbol{R}_{2} \in \mathbb{R}^{10,12}$ are given by

$$
\boldsymbol{R}_{1}(\boldsymbol{x}):=\left[\begin{array}{cccccccccc}
\gamma_{1} & 0 & 0 & 0 & 0 & 2 \beta_{3,2} & 4 \beta_{2} & 0 & 0 & 0 \\
\gamma_{1} & 0 & 0 & 2 \beta_{2,3} & 0 & 0 & 4 \beta_{3} & 0 & 0 & 0 \\
0 & \gamma_{2} & 0 & 2 \beta_{1,3} & 0 & 0 & 0 & 4 \beta_{3} & 0 & 0 \\
0 & \gamma_{2} & 0 & 0 & 2 \beta_{3,1} & 0 & 0 & 4 \beta_{1} & 0 & 0 \\
0 & 0 & \gamma_{3} & 0 & 2 \beta_{2,1} & 0 & 0 & 0 & 4 \beta_{1} & 0 \\
0 & 0 & \gamma_{3} & 0 & 0 & 2 \beta_{1,2} & 0 & 0 & 4 \beta_{2} & 0 \\
0 & 0 & 0 & 0 & 0 & 2 \beta_{3,2} & 4 \beta_{1,3} & 0 & 0 & -3 \gamma_{1} \\
0 & 0 & 0 & 2 \beta_{2,3} & 0 & 0 & 4 \beta_{1,2} & 0 & 0 & -3 \gamma_{1} \\
0 & 0 & 0 & 2 \beta_{1,3} & 0 & 0 & 0 & 4 \beta_{2,1} & 0 & -3 \gamma_{2} \\
0 & 0 & 0 & 0 & 2 \beta_{3,1} & 0 & 0 & 4 \beta_{2,3} & 0 & -3 \gamma_{2} \\
0 & 0 & 0 & 0 & 2 \beta_{2,1} & 0 & 0 & 0 & 4 \beta_{3,2} & -3 \gamma_{3} \\
0 & 0 & 0 & 0 & 0 & 2 \beta_{1,2} & 0 & 0 & 4 \beta_{3,1} & -3 \gamma_{3}
\end{array}\right]
$$

and

$$
\boldsymbol{R}_{2}(\boldsymbol{x})=\left[\begin{array}{cccccccccccc}
\gamma_{1} & 2 \beta_{2} & 0 & 0 & 0 & 0 & 0 & 0 & 0 & 0 & 0 & 2 \beta_{3} \\
0 & 0 & 0 & 2 \beta_{1} & \gamma_{2} & 2 \beta_{3} & 0 & 0 & 0 & 0 & 0 & 0 \\
0 & 0 & 0 & 0 & 0 & 0 & 0 & 2 \beta_{2} & \gamma_{3} & 2 \beta_{1} & 0 & 0 \\
0 & \beta_{1,3} & 3 \beta_{3} & \beta_{2,3} & 0 & 0 & 0 & 0 & 0 & 0 & 0 & 0 \\
0 & 0 & 0 & 0 & 0 & \beta_{2,1} & 3 \beta_{1} & \beta_{3,1} & 0 & 0 & 0 & 0 \\
0 & 0 & 0 & 0 & 0 & 0 & 0 & 0 & 0 & \beta_{3,2} & 3 \beta_{2} & \beta_{1,2} \\
0 & \frac{\beta_{1,3}}{2} & \frac{3 \beta_{2}}{2} & 0 & 0 & 0 & 0 & 0 & 0 & 0 & \frac{3 \beta_{3}}{2} & \frac{\beta_{1,2}}{2} \\
0 & 0 & \frac{3 \beta_{1}}{2} & \frac{\beta_{2,3}}{2} & 0 & \frac{\beta_{2,1}}{2} & \frac{3 \beta_{3}}{2} & 0 & 0 & 0 & 0 & 0 \\
0 & 0 & 0 & 0 & 0 & 0 & \frac{3 \beta_{2}}{2} & \frac{\beta_{3,1}}{2} & 0 & \frac{\beta_{3,2}}{2} & \frac{3 \beta_{1}}{2} & 0 \\
0 & 0 & -\gamma_{3} & 0 & 0 & 0 & -\gamma_{1} & 0 & 0 & 0 & -\gamma_{2} & 0
\end{array}\right] .
$$


Here $\boldsymbol{\beta}=\left[\beta_{1}, \beta_{2}, \beta_{3}\right]^{T}$ is the vector of barycentric coordinates of $\boldsymbol{x}$ with respect to the triangle $\Delta$ so that $\beta_{i}\left(\boldsymbol{p}_{j}\right)=\delta_{i, j}$ and

$$
\gamma_{j}:=2 \beta_{j}-1, \quad \beta_{i, j}=\beta_{i}-\beta_{j}, \text { for } i, j=1,2,3 .
$$

Moreover, $\boldsymbol{R}_{d}(i, j) S_{i, d-1}(\boldsymbol{x}) \geq 0$ for all $i, j$ and $\boldsymbol{x} \in \Delta$.

To prove this result we use the recurrence and knot insertion relations found in [17. The details of the proof can be found in Appendix A

Using (2.14) repeatedly we obtain,

Corollary 2.4. Suppose $\boldsymbol{x} \in \Delta_{k}$ for some $1 \leq k \leq 12$ and $0 \leq d \leq 2$. Then

$$
\boldsymbol{s}_{d}(\boldsymbol{x})^{T}:=\left[S_{1, d}(\boldsymbol{x}), \ldots, S_{n_{d}, d}(\boldsymbol{x})\right]=\boldsymbol{e}_{k}^{T} \prod_{i=1}^{d} \boldsymbol{R}_{i}(\boldsymbol{x}) .
$$

Moreover, for a spline with coefficients $\boldsymbol{c}=\left[c_{1}, \ldots, c_{n_{d}}\right]^{T}$,

$$
f_{d}(\boldsymbol{x}):=\sum_{j=1}^{n_{d}} c_{j} S_{j, d}(\boldsymbol{x})=\boldsymbol{e}_{k}^{T} \prod_{i=1}^{d} \boldsymbol{R}_{i}(\boldsymbol{x}) \boldsymbol{c} .
$$

We need to establish some notation before developing an analog of (2.11) for the S-spline basis.

Definition 2.5. We define

$$
\begin{aligned}
\mathcal{G}_{d}^{k} & :=\left\{j: \Delta_{k} \subset \operatorname{supp}\left(S_{j, d}\right)\right\}, \quad d=0,1,2, \\
\mathcal{H}_{d}^{k} & :=\left\{j: \boldsymbol{R}_{d}(k, j) \neq 0 \text { for some } \boldsymbol{x}\right\}, \quad k=1, \ldots, n_{d-1}, \quad d=1,2 .
\end{aligned}
$$

We use the symbols $\boldsymbol{g}_{d}^{k}$ and $\boldsymbol{h}_{d}^{k}$ for the vectors consisting of the elements in $\mathcal{G}_{d}^{k}$ and $\mathcal{H}_{d}^{k}$, respectively, arranged in increasing order.

The elements in $\mathcal{G}_{d}^{k}$ single out the indices of the S-splines of degree $d$ that are nonzero over triangle $\Delta_{k}$. It is easily verified that each $\mathcal{G}_{d}^{k}$ contains $\nu_{d}$ elements, so that $\boldsymbol{g}_{d}^{k}=\left[i_{1}, \ldots, i_{\nu_{d}}\right]^{T}$ with $i_{1} \leq \cdots \leq i_{\nu_{d}}$ (cf. Figures 4 and 5), and we recall that $\nu_{d}$ is the dimension of the polynomial space $\Pi_{d}\left(\mathbb{R}^{2}\right), d=0,1,2$. We show the elements in the sets $\mathcal{H}_{d}^{k}$ and $\mathcal{G}_{d}^{k}$ in Table 1

We also note that

$$
\mathcal{G}_{1}^{k}=\mathcal{H}_{1}^{k} \quad \text { and } \quad \mathcal{G}_{2}^{k}=\mathcal{H}_{2}^{i_{1}} \cup \mathcal{H}_{2}^{i_{2}} \cup \mathcal{H}_{2}^{i_{3}},\left[i_{1}, i_{2}, i_{3}\right]=\boldsymbol{g}_{1}^{k}, k=1, \ldots, 12 .
$$

Definition 2.6. For $d=0,1,2$ define $S_{j, d}^{k}$ to be the polynomial representing $S_{j, d}$ on $\Delta_{k}$. Also define,

$$
\tilde{\boldsymbol{s}}_{d}^{k}=\left[S_{1, d}^{k}, \ldots, S_{n_{d}, d}^{k}\right]^{T}, \quad \boldsymbol{s}_{d}^{k}=\tilde{\boldsymbol{s}}_{d}^{k}\left(\boldsymbol{g}_{d}^{k}\right) \in \mathbb{R}^{\nu_{d}},
$$

which represents the ordered vector whose elements are in the set $\mathcal{S}_{d}^{k}:=\left\{S_{j, d}^{k}: j \in\right.$ $\left.\mathcal{G}_{d}^{k}\right\}$.

Definition 2.7. For $1 \leq k \leq 12$ define submatrices

$$
\boldsymbol{R}_{1}^{k}:=\boldsymbol{R}_{1}\left(k, \boldsymbol{g}_{1}^{k}\right) \in \mathbb{R}^{1,3}, \quad \boldsymbol{R}_{2}^{k}:=\boldsymbol{R}_{2}\left(\boldsymbol{g}_{1}^{k}, \boldsymbol{g}_{2}^{k}\right) \in \mathbb{R}^{3,6},
$$

where $\boldsymbol{g}_{d}^{k}$ is defined in Definition 2.5. 
TABLE 1. Enumeration of the sets $\mathcal{H}_{d}^{k}$ and $\mathcal{G}_{d}^{k}$.

\begin{tabular}{|c|c|c|c|}
\hline$k$ & $\mathcal{H}_{1}^{k}=\mathcal{G}_{1}^{k}$ & $\mathcal{H}_{2}^{k}$ & $\mathcal{G}_{2}^{k}$ \\
\hline 1 & $1,6,7$ & $1,2,12$ & $1,2,3,10,11,12$ \\
\hline 2 & $1,4,7$ & $4,5,6$ & $1,2,3,4,11,12$ \\
\hline 3 & $2,4,8$ & $8,9,10$ & $2,3,4,5,6,7$ \\
\hline 4 & $2,5,8$ & $2,3,4$ & $3,4,5,6,7,8$ \\
\hline 5 & $3,5,9$ & $6,7,8$ & $6,7,8,9,10,11$ \\
\hline 6 & $3,6,9$ & $10,11,12$ & $7,8,9,10,11,12$ \\
\hline 7 & $6,7,10$ & $2,3,11,12$ & $2,3,7,10,11,12$ \\
\hline 8 & $4,7,10$ & $3,4,6,7$ & $2,3,4,7,11,12$ \\
\hline 9 & $4,8,10$ & $7,8,10,11$ & $2,3,4,6,7,11$ \\
\hline 10 & $5,8,10$ & $3,7,11$ & $3,4,6,7,8,11$ \\
\hline 11 & $5,9,10$ & & $3,6,7,8,10,11$ \\
\hline 12 & $6,9,10$ & & $3,7,8,10,11,12$ \\
\hline
\end{tabular}

For example, from Table[1it follows that $\boldsymbol{g}_{1}^{1}=[1,6,7]$ and $\boldsymbol{g}_{2}^{1}=[1,2,3,10,11,12]$. Thus,

$$
\boldsymbol{R}_{1}^{1}(\boldsymbol{x}) \boldsymbol{R}_{2}^{1}(\boldsymbol{x})=\left[\begin{array}{lll}
\gamma_{1} & 2 \gamma_{3,2} & 4 \beta_{2}
\end{array}\right]\left[\begin{array}{cccccc}
\gamma_{1} & 2 \beta_{2} & 0 & 0 & 0 & 2 \beta_{3} \\
0 & 0 & 0 & \gamma_{32} & 3 \beta_{2} & \gamma_{12} \\
0 & \frac{\gamma_{13}}{2} & \frac{3 \beta_{2}}{2} & 0 & \frac{3 \beta_{3}}{2} & \frac{\gamma_{12}}{2}
\end{array}\right]
$$

We are now ready to state the polynomial version of Corollary 2.4 (cf. equation (2.11) $)$.

Corollary 2.8. For $k=1, \ldots, 12, d=0,1,2$ and $\boldsymbol{c}^{T}=\left[c_{1}, \ldots, c_{n_{d}}\right]$,

$$
\boldsymbol{s}_{d}^{k T}=\prod_{i=1}^{d} \boldsymbol{R}_{i}^{k}, \quad f_{d}^{k}=\prod_{i=1}^{d} \boldsymbol{R}_{i}^{k} \boldsymbol{c}\left(\boldsymbol{g}_{d}^{k}\right), \quad d=0,1,2 .
$$

Here $\boldsymbol{c}\left(\boldsymbol{g}_{d}^{k}\right)$ is the vector $\left[c_{i_{1}}, \ldots, c_{i_{d}}\right]^{T}$, where $\boldsymbol{g}_{d}^{k}=\left[i_{1}, \ldots, i_{\nu_{d}}\right]^{T}$. Moreover, $f_{d}^{k}$ is the function $f_{d}$ restricted to triangle $\Delta_{k}$.

Proof. Clearly, $\boldsymbol{s}_{0}^{k}(\boldsymbol{x})=f_{0}^{k}(\boldsymbol{x})=1$ showing the result for $d=0$. By (2.18)

$$
S_{j, 1}^{k}=\boldsymbol{R}_{1}(k, j), \quad j=1, \ldots, 10,
$$

and (2.22) follows for $d=1$. For $j=1, \ldots, 12$

$$
\begin{aligned}
S_{j, 2}^{k} & =\boldsymbol{e}_{k}^{T} \boldsymbol{R}_{1} \boldsymbol{R}_{2}(:, j)=\sum_{i=1}^{10} \boldsymbol{R}_{1}(k, i) \boldsymbol{R}_{2}(i, j) \\
& =\sum_{i \in \mathcal{G}_{1}^{k}} \boldsymbol{R}_{1}(k, i) \boldsymbol{R}_{2}(i, j)=\boldsymbol{R}_{1}\left(k, \boldsymbol{g}_{1}^{k}\right) \boldsymbol{R}_{2}\left(\boldsymbol{g}_{1}^{k}, j\right) .
\end{aligned}
$$

But then (2.22) follows for $d=2$.

The edge property of Theorem 2.2 can now be proved. Suppose $f_{d}=\sum_{j=1}^{n_{d}} c_{j} S_{j, d}$ is a spline of degree $d$ on $\Delta_{P S 12}$ and $\boldsymbol{x}=(1-t) \boldsymbol{p}_{1}+t \boldsymbol{p}_{2}$. The corresponding barycentric coordinates are $\beta_{1}=1-t, \beta_{2}=t$, and $\beta_{3}=0$. Let $\left\{B_{j, 1}\right\}_{j=1}^{3}$ be the consecutive univariate linear B-splines on the knot vector $\left[0,0, \frac{1}{2}, 1,1\right]$, normalized 
to form a partition of unity on $[0,1]$. If $0 \leq t<1 / 2$ then $\boldsymbol{x} \in \Delta_{2}$, and (2.22) takes the form

$$
\begin{aligned}
f_{1}^{2}(\boldsymbol{x}) & =\boldsymbol{R}_{1}^{2}(\boldsymbol{x})\left[\begin{array}{lll}
c_{1} & c_{4} & c_{7}
\end{array}\right]^{T}=B_{1,1}(t) c_{1}+B_{2,1}(t) c_{4}, \\
\boldsymbol{R}_{1}^{2}(\boldsymbol{x}) & =\left[\begin{array}{lll}
1-2 t & 2 t & 0
\end{array}\right]
\end{aligned}
$$

while if $\frac{1}{2} \leq t \leq 1$, then $\boldsymbol{x} \in \Delta_{3}$ and

$$
\begin{aligned}
f_{1}^{3}(\boldsymbol{x}) & =\boldsymbol{R}_{1}^{3}(\boldsymbol{x})\left[\begin{array}{lll}
c_{2} & c_{4} & c_{8}
\end{array}\right]^{T}=B_{2,1}(t) c_{2}+B_{3,1}(t) c_{4}, \\
\boldsymbol{R}_{1}^{3}(\boldsymbol{x}) & =\left[\begin{array}{lll}
2 t-1 & 2-2 t & 0
\end{array}\right] .
\end{aligned}
$$

For $d=2$ and $\boldsymbol{x} \in \Delta_{2}$ let $\left\{B_{j, 2}\right\}_{j=1}^{4}$ be the consecutive univariate quadratic Bsplines on the knot vector $\left[0,0,0, \frac{1}{2}, 1,1,1\right]$, normalized to form a partition of unity on $[0,1]$. Then

$$
\begin{aligned}
f_{2}^{2}(\boldsymbol{x}) & =\boldsymbol{R}_{1}^{2}(\boldsymbol{x})\left[\begin{array}{cccccc}
1-2 t & 2 t & 0 & 0 & 0 & 0 \\
0 & 1-t & 0 & t & 0 & 0 \\
0 & \frac{1-t}{2} & \frac{3 t}{2} & 0 & 0 & \frac{1}{2}-t
\end{array}\right]\left[\begin{array}{llllll}
c_{1} & c_{2} & c_{3} & c_{4} & c_{11} & c_{12}
\end{array}\right]^{T} \\
& =(1-2 t)^{2} c_{1}+2 t(2-3 t) c_{2}+2 t^{2} c_{4}=B_{1,2}(t) c_{1}+B_{2,2}(t) c_{2}+B_{3,2}(t) c_{4},
\end{aligned}
$$

while for $\boldsymbol{x} \in \Delta_{3}$,

$$
\begin{aligned}
f_{2}^{3}(\boldsymbol{x}) & =\boldsymbol{R}_{1}^{3}(\boldsymbol{x})\left[\begin{array}{cccccc}
0 & 0 & 2-2 t & 2 t-1 & 0 & 0 \\
1-t & 0 & t & 0 & 0 & 0 \\
0 & \frac{3}{2}(1-t) & \frac{t}{2} & 0 & t-\frac{1}{2} & 0
\end{array}\right]\left[\begin{array}{c}
c_{2} \\
\vdots \\
c_{7}
\end{array}\right] \\
& =2(1-t)^{2} c_{2}+2(1-t)(3 t-1) c_{4}+(1-2 t)^{2} c_{5} \\
& =B_{2,2}(t) c_{2}+B_{3,2}(t) c_{4}+B_{4,2}(t) c_{5} .
\end{aligned}
$$

This proves the edge property.

\section{A Marsden-Like identity}

In the univariate B-spline case, the Marsden identity is usually written,

$$
(z-x)^{d}=\sum_{j} B_{j, d}(x) \prod_{k=j+1}^{j+d}\left(z-t_{k}\right),
$$

where $B_{j, d}$ is a B-spline of degree $d$ with knots $t_{j}, t_{j+1}, \ldots, t_{j+d+1}$, and $\left\{B_{j, d}\right\}_{j}$ is normalized to be a partition of unity. Dividing both sides by $z^{d}$ and setting $y:=z^{-1}$ we obtain a form more amenable to multivariate generalization

$$
(1-x y)^{d}=\sum_{j} B_{j, d}(x) \psi_{j, d}(y), \quad \psi_{j, d}(y)=\prod_{k=j+1}^{j+d}\left(1-t_{k} y\right) .
$$

The $\psi_{j, d}$ are polynomials of degree $\leq d$ called dual polynomials.

Consider now the S-spline basis. We define dual polynomials $\psi_{j, d}$ of degree $d$ for $j=1, \ldots, n_{d}$. For $d=0, \psi_{j, 0}=1$ and for $d>0$,

$$
\psi_{j, d}(\boldsymbol{y}):=\prod_{r=1}^{d}\left(1-\boldsymbol{p}_{j, d, r}^{* T} \boldsymbol{y}\right), \quad d=1,2, \quad \boldsymbol{y} \in \mathbb{R}^{2},
$$


where

$$
\begin{aligned}
\boldsymbol{p}_{j, 1,1}^{*} & :=\boldsymbol{p}_{j}, \quad j=1, \ldots, 10 \\
{\left[\boldsymbol{p}_{1,2,1}^{*}, \ldots, \boldsymbol{p}_{12,2,1}^{*}\right] } & :=\left[\boldsymbol{p}_{1}, \boldsymbol{p}_{1}, \boldsymbol{p}_{4}, \boldsymbol{p}_{4}, \boldsymbol{p}_{2}, \boldsymbol{p}_{2}, \boldsymbol{p}_{5}, \boldsymbol{p}_{5}, \boldsymbol{p}_{3}, \boldsymbol{p}_{3}, \boldsymbol{p}_{6}, \boldsymbol{p}_{6}\right] \\
{\left[\boldsymbol{p}_{1,2,2}^{*}, \ldots, \boldsymbol{p}_{12,2,2}^{*}\right] } & :=\left[\boldsymbol{p}_{1}, \boldsymbol{p}_{4}, \boldsymbol{p}_{10}, \boldsymbol{p}_{2}, \boldsymbol{p}_{2}, \boldsymbol{p}_{5}, \boldsymbol{p}_{10}, \boldsymbol{p}_{3}, \boldsymbol{p}_{3}, \boldsymbol{p}_{6}, \boldsymbol{p}_{10}, \boldsymbol{p}_{1}\right]
\end{aligned}
$$

are called dual points. There are no dual points for $d=0$.

Theorem 3.1 (Marsden-like Identity). For $d=0,1,2$

$$
\left(1-\boldsymbol{x}^{T} \boldsymbol{y}\right)^{d}=\sum_{j=1}^{n_{d}} S_{j, d}(\boldsymbol{x}) \psi_{j, d}(\boldsymbol{y})=\boldsymbol{s}_{d}(\boldsymbol{x})^{T} \boldsymbol{\psi}_{d}(\boldsymbol{y}), \quad \boldsymbol{x} \in \Delta, \boldsymbol{y} \in \mathbb{R}^{2},
$$

where

$$
\boldsymbol{s}_{d}(\boldsymbol{x})^{T}:=\left[S_{1, d}(\boldsymbol{x}), \ldots, S_{n_{d}, d}(\boldsymbol{x})\right], \quad \boldsymbol{\psi}_{d}(\boldsymbol{y}):=\left[\psi_{1, d}(\boldsymbol{y}), \ldots, \psi_{n_{d}, d}(\boldsymbol{y})\right]^{T} .
$$

Also, there are 12 polynomial versions, one for each subtriangle $\Delta_{k}, k=1, \ldots, 12$. If $\boldsymbol{g}_{d}^{k}:=\left[i_{1}, \ldots, i_{\nu_{d}}\right]^{T}$ is given as in Definition 2.5 , then

$$
\left(1-\boldsymbol{x}^{T} \boldsymbol{y}\right)^{d}=\sum_{j=1}^{\nu_{d}} S_{i_{j}, d}^{k}(\boldsymbol{x}) \psi_{i_{j}, d}(\boldsymbol{y})=\boldsymbol{s}_{d}^{k}(\boldsymbol{x})^{T} \boldsymbol{\psi}_{d}^{k}(\boldsymbol{y}), \quad k=1, \ldots, 12, \quad \boldsymbol{x}, \boldsymbol{y} \in \mathbb{R}^{2} .
$$

Here $S_{j, d}^{k}$ is the polynomial representing $S_{j, d}$ on the subtriangle $\Delta_{k}$ and

$$
\boldsymbol{s}_{d}^{k}(\boldsymbol{x})^{T}:=\left[S_{i_{1}, d}^{k}(\boldsymbol{x}), \ldots, S_{i_{\nu_{d}}, d}^{k}(\boldsymbol{x})\right], \quad \boldsymbol{\psi}_{d}^{k}(\boldsymbol{y}):=\left[\psi_{i_{1}, d}(\boldsymbol{y}), \ldots, \psi_{i_{\nu_{d}}, d}(\boldsymbol{y})\right]^{T} .
$$

Note the similarity between equation (3.1) and equation (3.4). In the linear case the term $1-t_{j+1} y$ is replaced by $1-\boldsymbol{p}_{j}^{T} \boldsymbol{y}$, and, in the quadratic case, we move around the boundary of $\Delta$ inserting $\boldsymbol{p}_{10}$ for the $S_{j, 2}$ with trapezoidal support. In contrast to the univariate case, the dual points are not always knots of the S-splines. In particular, $\boldsymbol{p}_{10}$ is not a knot of any quadratic S-spline.

The Marsden-like identity can be used to give explicit representations for monomials.

Lemma 3.2. For $\boldsymbol{x}=\left(x_{1}, x_{2}\right) \in \Delta$,

$$
\begin{aligned}
1 & =\sum_{j=1}^{n_{d}} S_{j, d}(\boldsymbol{x}), \quad d=0,1,2, \\
\boldsymbol{x} & =\sum_{j=1}^{n_{d}} \boldsymbol{m}_{j, d} S_{j, d}(\boldsymbol{x}), \quad d=1,2, \\
{\left[\begin{array}{cc}
2 x_{1}^{2} & x_{1} x_{2} \\
x_{2} x_{1} & 2 x_{2}^{2}
\end{array}\right] } & =\sum_{j=1}^{12} \boldsymbol{H}\left(\psi_{j, 2}\right) S_{j, 2}(\boldsymbol{x}),
\end{aligned}
$$

where

$$
\begin{aligned}
& \boldsymbol{m}_{j, 1}:=\boldsymbol{p}_{j, 1,1}^{*}=\boldsymbol{p}_{j}, \quad j=1,2, \ldots, 10, \\
& \boldsymbol{m}_{j, 2}:=\left(\boldsymbol{p}_{j, 2,1}^{*}+\boldsymbol{p}_{j, 2,2}^{*}\right) / 2, \quad j=1,2, \ldots, 12,
\end{aligned}
$$

and $\boldsymbol{p}_{j, 2, r}^{*}$ are given by (3.3). Moreover,

$$
\boldsymbol{H}\left(\psi_{j, 2}\right):=\left[\begin{array}{cc}
D_{\boldsymbol{e}_{1}}^{2}\left(\psi_{j, 2}\right) & D_{\boldsymbol{e}_{1}} D_{\boldsymbol{e}_{2}}\left(\psi_{j, 2}\right) \\
D_{\boldsymbol{e}_{2}} D_{\boldsymbol{e}_{1}}\left(\psi_{j, 2}\right) & D_{\boldsymbol{e}_{2}}^{2}\left(\psi_{j, 2}\right)
\end{array}\right]
$$


Proof. We set $\boldsymbol{y}=\mathbf{0}$ in equation (3.4) to get,

$$
1=\boldsymbol{s}_{d}(\boldsymbol{x})^{T} \boldsymbol{\psi}_{d}(\mathbf{0})=\boldsymbol{s}_{d}(\boldsymbol{x})^{T} \boldsymbol{e}=\sum_{j=1}^{n_{d}} S_{j, d}(\boldsymbol{x})
$$

which proves Property 3 in Theorem 2.2, the partition of unity normalization. To show the expression for $\boldsymbol{x}$ in (3.7), we differentiate equation (3.4) with respect to $\boldsymbol{y}$, and set $\boldsymbol{y}=\mathbf{0}$ so,

$$
-d \boldsymbol{x}^{T}=\boldsymbol{s}_{d}(\boldsymbol{x})^{T} \nabla_{\boldsymbol{y}} \boldsymbol{\psi}_{d}(\mathbf{0}),
$$

where $\nabla_{\boldsymbol{y}} \boldsymbol{\psi}_{d}(\boldsymbol{y})$ is the $n_{d} \times 2$ matrix whose $r$ th column is $\partial \boldsymbol{\psi}_{d}(\boldsymbol{y}) / \partial y_{r}, r=1,2$. Differentiating and setting $\boldsymbol{y}=\mathbf{0}$ lead to,

$$
\begin{aligned}
-\boldsymbol{x}^{T} & =-\boldsymbol{s}_{1}(\boldsymbol{x})^{T}\left[\boldsymbol{p}_{1,1,1}^{*}, \ldots, \boldsymbol{p}_{10,1,1}^{*}\right]^{T}, \\
-2 \boldsymbol{x}^{T} & =-\boldsymbol{s}_{2}(\boldsymbol{x})^{T}\left[\boldsymbol{p}_{1,2,1}^{*}+\boldsymbol{p}_{1,2,2}^{*}, \ldots, \boldsymbol{p}_{12,2,1}^{*}+\boldsymbol{p}_{12,2,2}^{*}\right]^{T},
\end{aligned}
$$

from whence the identity for $\boldsymbol{x}$ follows.

Applying second order partial derivatives with respect to $\boldsymbol{y}$ on both sides of equation (3.4) and setting $\boldsymbol{y}=\mathbf{0}$, we obtain the last equation.

Definition 3.3. The points $\boldsymbol{m}_{j, d}$ given by (3.8) for $j=1, \ldots, n_{d}$ are called domain points. The domain mesh $\mathcal{M}_{d}(\Delta)$ is defined by vertices and edges. Two domain points $\boldsymbol{m}_{j, 2}=\left(\boldsymbol{p}_{j, 2,1}^{*}+\boldsymbol{p}_{j, 2,2}^{*}\right) / 2$ and $\boldsymbol{m}_{k, 2}=\left(\boldsymbol{p}_{k, 2,1}^{*}+\boldsymbol{p}_{k, 2,2}^{*}\right) / 2$ define an edge if $\left\{\boldsymbol{p}_{j, 2,1}^{*}, \boldsymbol{p}_{j, 2,2}^{*}\right\} \cap\left\{\boldsymbol{p}_{k, 2,1}^{*}, \boldsymbol{p}_{k, 2,2}^{*}\right\} \neq \emptyset$. Alternatively,

$$
\mathcal{M}_{d}(\Delta):=\bigcup_{i=1}^{n_{d-1}} \partial \operatorname{ch}\left(\left\{\boldsymbol{m}_{j, d}: j \in \mathcal{H}_{d}^{i}\right\}\right),
$$

where $\mathcal{H}_{d}^{i}$ is given in Definition 2.5 and $\partial \mathrm{ch}$ is the boundary of the convex hull of $\left\{\boldsymbol{m}_{j, d}: j \in \mathcal{H}_{d}^{i}\right\}$.

A spline $f_{d}:=\sum_{j=1}^{n_{d}} c_{j} S_{j, d}$ has control points

$$
\gamma_{j, d}:=\left(\boldsymbol{m}_{j, d}, c_{j}\right), \quad j=1, \ldots, n_{d},
$$

where the $\boldsymbol{m}_{j, d}$ are the domain points given by (3.8). The control mesh $\boldsymbol{\Gamma}_{d}(\Delta, \boldsymbol{c})$ is defined by lifting the domain mesh by a piecewise bilinear mapping $A: \mathcal{M}_{d}(\Delta) \rightarrow$ $\boldsymbol{\Gamma}_{d}(\Delta, \boldsymbol{c})$ defined so that $A\left(\boldsymbol{m}_{j, d}\right)=\gamma_{j, d}$ for all $j$.

The edges of $\mathcal{M}_{1}(\Delta)$ are simply the mesh lines in $\Delta_{P S 12}$ as shown in Figure 2, The domain mesh and the control mesh for $d=2$ are shown in Figure 7 The domain mesh defined in Definition 3.3 is also related to the pyramidal Algorithm $4.1(d=2, r=0)$ as shown in Figure 8. It is unusual in that it is a hybrid mesh comprised of both triangular and quadrilateral faces.

In the univariate B-spline case, the numbers used to represent $x$ in terms of Bsplines are known as nodes, knot averages or Greville points. For $\Delta_{P S 12}$ they could analogously be called dual point averages or Greville points. Following the terminology used in [13, we shall call them domain points.

3.1. Proof of the Marsden-like identity. The same matrices $\boldsymbol{R}_{d}$ introduced in subsection 2.1 for recurrence relations for S-splines also appear in recurrence relations for the dual polynomials. 


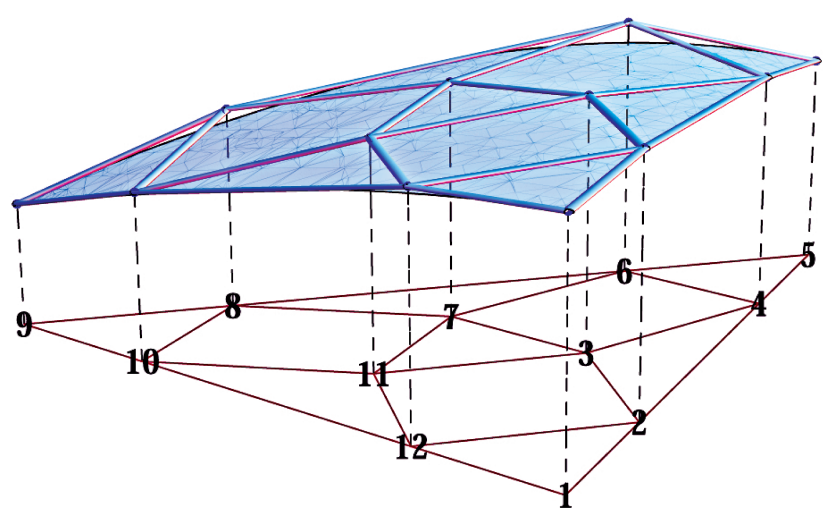

FIGURE 7. Quadratic domain mesh $\mathcal{M}_{2}(\Delta)$ and the corresponding control mesh for some surface $f$.

Theorem 3.4. For $\boldsymbol{x}, \boldsymbol{y} \in \mathbb{R}^{2}$, and $d=1,2$,

$$
\begin{aligned}
& \boldsymbol{R}_{d}(\boldsymbol{x}) \boldsymbol{\psi}_{d}(\boldsymbol{y})=\left(1-\boldsymbol{x}^{T} \boldsymbol{y}\right) \boldsymbol{\psi}_{d-1}(\boldsymbol{y}) \\
& \boldsymbol{R}_{d}^{k}(\boldsymbol{x}) \boldsymbol{\psi}_{d}^{k}(\boldsymbol{y})=\left(1-\boldsymbol{x}^{T} \boldsymbol{y}\right) \boldsymbol{\psi}_{d-1}^{k}(\boldsymbol{y}), \quad k=1, \ldots, 12
\end{aligned}
$$

where, $\boldsymbol{R}_{1}$ and $\boldsymbol{R}_{2}$ are given by (2.15) and (2.16), $\boldsymbol{\psi}_{0}, \boldsymbol{\psi}_{1}, \boldsymbol{\psi}_{2}$ by (3.2), $\boldsymbol{R}_{1}^{k}$ and $\boldsymbol{R}_{2}^{k}$ in Definition 2.7, and $\boldsymbol{\psi}_{d}^{k}=\boldsymbol{\psi}_{d}\left(\boldsymbol{g}_{d}^{k}\right)$. Moreover, $\boldsymbol{g}_{d}^{k}$ is given in Definition 2.5.

Proof. Let $\ell_{j}=\ell_{j}(\boldsymbol{y})=1-\boldsymbol{p}_{j}^{T} \boldsymbol{y}$ for $\boldsymbol{y} \in \mathbb{R}^{2}$. Fix $\boldsymbol{x}, \boldsymbol{y} \in \mathbb{R}^{2}$ and let $\boldsymbol{\beta}$ be the vector of barycentric coordinates of $\boldsymbol{x}$ with respect to $\Delta$. Then

$$
1-\boldsymbol{x}^{T} \boldsymbol{y}=\left(\beta_{1}+\beta_{2}+\beta_{3}\right)-\left(\beta_{1} \boldsymbol{p}_{1}+\beta_{2} \boldsymbol{p}_{2}+\beta_{3} \boldsymbol{p}_{3}\right)^{T} \boldsymbol{y}=\beta_{1} \ell_{1}+\beta_{2} \ell_{2}+\beta_{3} \ell_{3} .
$$

Consider first $d=1$. Because of the symmetry of the equations for the boundary triangles and analogous similarity for the interior triangles, we consider only a single outer triangle $\Delta_{1}$ corresponding to $\psi_{1,0}$ and a single interior triangle $\Delta_{7}$ corresponding to $\psi_{7,0}$. Combining (2.15), (1.2), (3.14) and (3.2) we obtain,

$$
\begin{aligned}
\left(\boldsymbol{R}_{1}(\boldsymbol{x}) \boldsymbol{\psi}_{1}(\boldsymbol{y})\right)_{1} & =\gamma_{1} \ell_{1}+2 \beta_{32} \ell_{6}+4 \beta_{2} \ell_{7} \\
& =\left(2 \beta_{1}-1\right) \ell_{1}+\left(\beta_{3}-\beta_{2}\right)\left(\ell_{1}+\ell_{3}\right)+\beta_{2}\left(2 \ell_{1}+\ell_{2}+\ell_{3}\right) \\
& =\beta_{1} \ell_{1}+\beta_{2} \ell_{2}+\beta_{3} \ell_{3}=\left(1-\boldsymbol{x}^{T} \boldsymbol{y}\right) \psi_{1,0},
\end{aligned}
$$

and

$$
\begin{aligned}
\left(\boldsymbol{R}_{1}(\boldsymbol{x}) \boldsymbol{\psi}_{1}(\boldsymbol{y})\right)_{7} & =2 \beta_{32} \ell_{6}+4 \beta_{13} \ell_{7}-3 \gamma_{1} \ell_{10} \\
& =\left(\beta_{3}-\beta_{2}\right)\left(\ell_{1}+\ell_{3}\right)+\left(\beta_{1}-\beta_{3}\right)\left(2 \ell_{1}+\ell_{2}+\ell_{3}\right) \\
& -\left(2 \beta_{1}-1\right)\left(\ell_{1}+\ell_{2}+\ell_{3}\right) \\
& =\beta_{1} \ell_{1}+\beta_{2} \ell_{2}+\beta_{3} \ell_{3}=\left(1-\boldsymbol{x}^{T} \boldsymbol{y}\right) \psi_{7,0} .
\end{aligned}
$$


For $d=2$ we consider rows 1, 4,7,10. Again, from (2.16), (1.2), (3.14), and (3.2), row 1 becomes

$$
\begin{aligned}
\left(\boldsymbol{R}_{2}(\boldsymbol{x}) \boldsymbol{\psi}_{2}(\boldsymbol{y})\right)_{1} & =\gamma_{1} \psi_{1,2}+2 \beta_{2} \psi_{2,2}+2 \beta_{3} \psi_{12,2} \\
& =\gamma_{1} \ell_{1}^{2}+2 \beta_{2} \ell_{1} \ell_{4}+2 \beta_{3} \ell_{6} \ell_{1} \\
& =\left(\left(2 \beta_{1}-1\right) \ell_{1}+\beta_{2}\left(\ell_{1}+\ell_{2}\right)+\beta_{3}\left(\ell_{3}+\ell_{1}\right)\right) \ell_{1} \\
& =\left(\beta_{1} \ell_{1}+\beta_{2} \ell_{2}+\beta_{3} \ell_{3}\right) \ell_{1}=\left(1-\boldsymbol{x}^{T} \boldsymbol{y}\right) \psi_{1,1},
\end{aligned}
$$

and, row 4 becomes

$$
\begin{aligned}
\left(\boldsymbol{R}_{2}(\boldsymbol{x}) \boldsymbol{\psi}_{2}(\boldsymbol{y})\right)_{4} & =\beta_{1,3} \psi_{2,2}+3 \beta_{3} \psi_{3,2}+\beta_{2,3} \psi_{4,2} \\
& =\beta_{1,3} \ell_{1} \ell_{4}+3 \beta_{3} \ell_{4} \ell_{10}+\beta_{2,3} \ell_{4} \ell_{2} \\
& \left.=\left(\left(\beta_{1}-\beta_{3}\right) \ell_{1}+\beta_{3}\left(\ell_{1}+\ell_{2}+\ell_{3}\right)+\left(\beta_{2}-\beta_{3}\right) \ell_{2}\right)\right) \ell_{4} \\
& =\left(\beta_{1} \ell_{1}+\beta_{2} \ell_{2}+\beta_{3} \ell_{3}\right) \ell_{4}=\left(1-\boldsymbol{x}^{T} \boldsymbol{y}\right) \psi_{4,1} .
\end{aligned}
$$

Inserting $\ell_{7}=\frac{1}{4}\left(2 \ell_{1}+\ell_{2}+\ell_{3}\right)$ for row 7 , we get

$$
\begin{aligned}
\left(\boldsymbol{R}_{2}(\boldsymbol{x}) \boldsymbol{\psi}_{2}(\boldsymbol{y})\right)_{7} & =\left(\beta_{13} \psi_{2,2}+3 \beta_{2} \psi_{3,2}+3 \beta_{3} \psi_{11,2}+\beta_{12} \psi_{12,2}\right) / 2 \\
& =\left(\beta_{13} \ell_{1} \ell_{4}+3 \beta_{2} \ell_{4} \ell_{10}+3 \beta_{3} \ell_{6} \ell_{10}+\beta_{12} \ell_{6} \ell_{1}\right) / 2 \\
& =\left(\beta_{1}\left(\ell_{4}+\ell_{6}\right) \ell_{1}+\beta_{2}\left(\left(\ell_{1}+\ell_{2}+\ell_{3}\right) \ell_{4}-\ell_{1} \ell_{6}\right)\right. \\
& \left.+\beta_{3}\left(\left(\ell_{1}+\ell_{2}+\ell_{3}\right) \ell_{6}-\ell_{1} \ell_{4}\right)\right) / 2 \\
& =\left(\beta_{1} \ell_{1}+\beta_{2} \ell_{2}+\beta_{3} \ell_{3}\right) \ell_{7}=\left(1-\boldsymbol{x}^{T} \boldsymbol{y}\right) \psi_{7,1}
\end{aligned}
$$

and, for row 10, we produce,

$$
\begin{aligned}
\left(\boldsymbol{R}_{2}(\boldsymbol{x}) \boldsymbol{\psi}_{2}(\boldsymbol{y})\right)_{10} & =-\gamma_{3} \psi_{3,2}-\gamma_{1} \psi_{7,2}-\gamma_{2} \psi_{11,2} \\
& =-\gamma_{3} \ell_{4} \ell_{10}-\gamma_{1} \ell_{5} \ell_{10}-\gamma_{2} \ell_{6} \ell_{10} \\
& =\left(\left(1-2 \beta_{3}\right)\left(\ell_{1}+\ell_{2}\right)+\left(1-2 \beta_{1}\right)\left(\ell_{2}+\ell_{3}\right)\right. \\
& \left.+\left(1-2 \beta_{2}\right)\left(\ell_{1}+\ell_{3}\right)\right) \ell_{10} / 2 \\
& =\left(\beta_{1} \ell_{1}+\beta_{2} \ell_{2}+\beta_{3} \ell_{3}\right) \ell_{10}=\left(1-\boldsymbol{x}^{T} \boldsymbol{y}\right) \psi_{10,1} .
\end{aligned}
$$

Next consider (3.13). Since $\boldsymbol{R}_{1}(k, j)=0$ for $j \notin \mathcal{G}_{1}^{k}$, it easily follows that

$$
\boldsymbol{R}_{1}^{k}(\boldsymbol{x}) \boldsymbol{\psi}_{1}^{k}(\boldsymbol{y})=\boldsymbol{e}_{k}^{T} \boldsymbol{R}_{1}(\boldsymbol{x}) \boldsymbol{\psi}_{1}(\boldsymbol{y})=\boldsymbol{e}_{k}^{T}\left(1-\boldsymbol{x}^{T} \boldsymbol{y}\right) \boldsymbol{\psi}_{0}(\boldsymbol{y})=\left(1-\boldsymbol{x}^{T} \boldsymbol{y}\right) \boldsymbol{\psi}_{0}^{k}(\boldsymbol{y}) .
$$

Finally, consider $d=2$. Let $\mathcal{G}_{1}^{k}=\left\{i_{1}, i_{2}, i_{3}\right\}$. Since $\boldsymbol{R}_{2}\left(i_{r}, j\right)=0$ for $j \notin \mathcal{H}_{2}^{i_{r}} \subset \mathcal{G}_{2}^{k}$ for $r=1,2,3$,

$$
\boldsymbol{R}_{2}^{k}(\boldsymbol{x}) \boldsymbol{\psi}_{2}^{k}(\boldsymbol{y})=\left[\begin{array}{c}
\boldsymbol{e}_{i_{1}}^{T} \\
\boldsymbol{e}_{i_{2}}^{T} \\
\boldsymbol{e}_{i_{3}}^{T}
\end{array}\right] \boldsymbol{R}_{2}(\boldsymbol{x}) \boldsymbol{\psi}_{2}(\boldsymbol{y})=\left[\begin{array}{c}
\boldsymbol{e}_{i_{1}}^{T} \\
\boldsymbol{e}_{i_{2}}^{T} \\
\boldsymbol{e}_{i_{3}}^{T}
\end{array}\right]\left(1-\boldsymbol{x}^{T} \boldsymbol{y}\right) \boldsymbol{\psi}_{1}(\boldsymbol{y})=\left(1-\boldsymbol{x}^{T} \boldsymbol{y}\right) \boldsymbol{\psi}_{1}^{k}(\boldsymbol{y})
$$

Invoking the recurrence relations for the dual polynomials we arrive at:

Theorem 3.5. For $d=1,2, \boldsymbol{x}_{j} \in \mathbb{R}^{2}, j=1, \ldots, d$ and $\boldsymbol{y} \in \mathbb{R}^{2}$,

$$
\left(1-\boldsymbol{x}_{1}^{T} \boldsymbol{y}\right) \cdots\left(1-\boldsymbol{x}_{d}^{T} \boldsymbol{y}\right) \boldsymbol{\psi}_{0}(\boldsymbol{y})=\boldsymbol{R}_{1}\left(\boldsymbol{x}_{1}\right) \cdots \boldsymbol{R}_{d}\left(\boldsymbol{x}_{d}\right) \boldsymbol{\psi}_{d}(\boldsymbol{y}) .
$$

Moreover,

$$
\left(1-\boldsymbol{x}_{1}^{T} \boldsymbol{y}\right) \cdots\left(1-\boldsymbol{x}_{d}^{T} \boldsymbol{y}\right)=\boldsymbol{R}_{1}^{k}\left(\boldsymbol{x}_{1}\right) \cdots \boldsymbol{R}_{d}^{k}\left(\boldsymbol{x}_{d}\right) \boldsymbol{\psi}_{d}^{k}(\boldsymbol{y}), k=1, \ldots, 12,
$$


where $\boldsymbol{R}_{d}^{k}$ is given in Definition 2.7 and $\boldsymbol{\psi}_{d}^{k}(\boldsymbol{y})$ by (3.6).

Proof. We simply apply (3.12) and (3.13) repeatedly.

Proof of Theorem 3.1. We use (3.15) and (3.16) with $\boldsymbol{x}_{1}=\cdots=\boldsymbol{x}_{d}=\boldsymbol{x}$. If $\boldsymbol{x} \in \Delta$, then $\boldsymbol{x} \in \Delta_{k}$ for some $k$. Using the $k$ th component of (3.15) we find, using Corollary 2.4, that

$$
\left(1-\boldsymbol{x}^{T} \boldsymbol{y}\right)^{d}=\boldsymbol{e}_{k}^{T} \prod_{i=1}^{d} \boldsymbol{R}_{i}(\boldsymbol{x}) \boldsymbol{\psi}_{d}(\boldsymbol{y})=\boldsymbol{s}_{d}(\boldsymbol{x})^{T} \boldsymbol{\psi}_{d}(\boldsymbol{y}) .
$$

This proves (3.4), and then, by (3.16) and (2.22),

$$
\left(1-\boldsymbol{x}^{T} \boldsymbol{y}\right)^{d}=\prod_{i=1}^{d} \boldsymbol{R}_{i}^{k}(\boldsymbol{x}) \boldsymbol{\psi}_{d}^{k}(\boldsymbol{y})=\boldsymbol{s}_{d}^{k}(\boldsymbol{x})^{T} \boldsymbol{\psi}_{d}^{k}(\boldsymbol{y}) .
$$

3.2. Linear independence. In this section we show linear independence of both the S-splines and subsets of the dual polynomials. Recall in our notation that $S_{j, d}^{k}$ is the polynomial that represents $S_{j, d}$ on $\Delta_{k}$ and that $S_{j, d}^{k}=0$ if $j \notin \mathcal{G}_{d}^{k}$.

Theorem 3.6 (Local linear independence). Suppose $d=0,1,2$ and $1 \leq k \leq 12$. Then pairs of sets $\mathcal{S}_{d}^{k}:=\left\{S_{j, d}^{k}: j \in \mathcal{G}_{d}^{k}\right\}$ and $\boldsymbol{\Psi}_{d}^{k}:=\left\{\psi_{j, d}: j \in \mathcal{G}_{d}^{k}\right\}$ are both bases for $\Pi_{d}\left(\mathbb{R}^{2}\right)$.

Proof. The fact that $\mathcal{S}_{d}^{k}$ forms a basis for $\Pi_{d}\left(\mathbb{R}^{2}\right)$ follows directly from conjoining the two requisite conditions, namely, Lemma 3.2 affords the spanning property for the set $\mathcal{S}_{d}^{k}$, and their linear independence follows from the observation that the set $\mathcal{G}_{d}^{k}$ contains exactly $\nu_{d}$ elements.

We now show that $\boldsymbol{\Psi}_{d}^{k}$ is also a basis. Inasmuch as $\boldsymbol{\Psi}_{d}^{k}$ has the same cardinality as $\Pi_{d}\left(\mathbb{R}^{2}\right)$, it remains only to show that $\boldsymbol{\Psi}_{d}^{k}$ spans $\Pi_{d}\left(\mathbb{R}^{2}\right)$. Setting $\boldsymbol{x}=\mathbf{0}$ in equation (3.5) yields $1=\boldsymbol{s}_{d}^{k}(\mathbf{0})^{T} \boldsymbol{\psi}_{d}^{k}(\boldsymbol{y})$, so the constant 1 can be expressed as a linear combination of $\psi$ 's. For $d>0$, differentiating (3.5) with respect to $\boldsymbol{x}$ and then setting $\boldsymbol{x}=\mathbf{0}$ to get $-d \boldsymbol{y}=\nabla_{\boldsymbol{x}} \boldsymbol{s}_{d}^{k}(0)^{T} \boldsymbol{\psi}_{d}^{k}(y)$, establishes that $\boldsymbol{y}$ is in the span of the $\psi$ 's. Taking the Hessian with respect to $\boldsymbol{x}$ on either side of equation (3.5) and setting $\boldsymbol{x}=\mathbf{0}$, we obtain the quadratic case.

Theorem 3.7. For $d=0,1,2$, the set of $S$-splines $\left\{S_{j, d}\right\}_{j=1}^{n_{d}}$ forms a basis for $\mathbb{S}_{d}$. Proof. Let $n_{d}=\operatorname{dim}\left(\mathbb{S}_{d}\right)$. By Theorem 2.2 the $n_{d}$ functions $S_{j, d}$ belong to $\mathbb{S}_{d}$. The result then follows as a consequence of local linear independence.

Marsden's identity and linear independence also imply the following symmetry property.

Lemma 3.8. For $\boldsymbol{x}, \boldsymbol{z} \in \mathbb{R}^{2}$,

$$
\begin{aligned}
& \boldsymbol{R}_{1}^{k}(\boldsymbol{x}) \boldsymbol{R}_{2}^{k}(\boldsymbol{z})=\boldsymbol{R}_{1}^{k}(\boldsymbol{z}) \boldsymbol{R}_{2}^{k}(\boldsymbol{x}), \quad k=1, \ldots, 12, \\
& \boldsymbol{R}_{1}(\boldsymbol{x}) \boldsymbol{R}_{2}(\boldsymbol{z})=\boldsymbol{R}_{1}(\boldsymbol{z}) \boldsymbol{R}_{2}(\boldsymbol{x}) .
\end{aligned}
$$

Proof. For any $\boldsymbol{x}, \boldsymbol{y}, \boldsymbol{z} \in \mathbb{R}^{2}$ and $1 \leq k \leq 12$,

$$
\begin{aligned}
\boldsymbol{R}_{1}^{k}(\boldsymbol{x}) \boldsymbol{R}_{2}^{k}(\boldsymbol{z}) \boldsymbol{\psi}_{2}^{k}(\boldsymbol{y}) & =\left(1-\boldsymbol{z}^{T} \boldsymbol{y}\right) \boldsymbol{R}_{1}^{k}(\boldsymbol{x}) \boldsymbol{\psi}_{1}^{k}(\boldsymbol{y})=\left(1-\boldsymbol{z}^{T} \boldsymbol{y}\right)\left(1-\boldsymbol{x}^{T} \boldsymbol{y}\right) \boldsymbol{\psi}_{0}^{k}(\boldsymbol{y}) \\
& =\left(1-\boldsymbol{x}^{T} \boldsymbol{y}\right)\left(1-\boldsymbol{z}^{T} \boldsymbol{y}\right) \boldsymbol{\psi}_{0}^{k}(\boldsymbol{y})=\left(1-\boldsymbol{x}^{T} \boldsymbol{y}\right) \boldsymbol{R}_{1}^{k}(\boldsymbol{z}) \boldsymbol{\psi}_{1}^{k}(\boldsymbol{y}) \\
& =\boldsymbol{R}_{1}^{k}(\boldsymbol{z}) \boldsymbol{R}_{2}^{k}(\boldsymbol{x}) \boldsymbol{\psi}_{2}^{k}(\boldsymbol{y}) .
\end{aligned}
$$


Since $\boldsymbol{\psi}_{2}^{k}$ is a basis for $\Pi_{2}\left(\mathbb{R}^{2}\right)$, (3.17) follows. Furthermore, for $\boldsymbol{x}, \boldsymbol{z} \in \Delta_{k}$, (3.18) follows from (3.17). Thus, (3.18) holds for all $\boldsymbol{x}, \boldsymbol{z} \in \mathbb{R}^{2}$.

3.3. Differentiation. In this section we derive a formula for the directional derivative of the S-splines $S_{j, d}$. Let $D_{\boldsymbol{u}}:=\boldsymbol{u} \cdot \nabla=u_{1} \frac{\partial}{\partial x_{1}}+u_{2} \frac{\partial}{\partial x_{2}}$ with $\boldsymbol{u}=\left[u_{1}, u_{2}\right]^{T}$ be a directional derivative. The unique solution $\boldsymbol{\alpha}:=\left[\alpha_{1}, \alpha_{2}, \alpha_{3}\right]^{T}$ of

$$
\begin{aligned}
& \alpha_{1}+\alpha_{2}+\alpha_{3}=0, \\
& \alpha_{1} \boldsymbol{p}_{1}+\alpha_{2} \boldsymbol{p}_{2}+\alpha_{3} \boldsymbol{p}_{3}=\boldsymbol{u}
\end{aligned}
$$

is called the directional coordinates of $\boldsymbol{u}$. If $\boldsymbol{u}=\boldsymbol{q}_{1}-\boldsymbol{q}_{2}$, with $\boldsymbol{q}_{i} \in \mathbb{R}^{2}$ for $i=1,2$ then $\alpha_{j}:=\beta_{1, j}-\beta_{2, j}, j=1,2,3$, where $\boldsymbol{\beta}^{i}:=\left[\beta_{i, 1}, \beta_{i, 2}, \beta_{i, 3}\right]^{T}$ is the vector of barycentric coordinates of $\boldsymbol{q}_{i}, i=1,2$.

We have the following differentiation formula:

Theorem 3.9. If $\boldsymbol{u} \in \mathbb{R}^{2}$ has directional coordinates $\boldsymbol{\alpha}$, then for $d=1,2$

$$
\begin{aligned}
D_{\boldsymbol{u}} \boldsymbol{s}_{d}^{T} & =d \boldsymbol{s}_{d-1}^{T} \boldsymbol{U}_{d, \boldsymbol{u}}, \\
D_{\boldsymbol{u}} \boldsymbol{s}_{d}^{k T} & =d \boldsymbol{s}_{d-1}^{k T} \boldsymbol{U}_{d, \boldsymbol{u}}^{k}, \quad k=1, \ldots, 12,
\end{aligned}
$$

where, with $\alpha_{i, j}:=\alpha_{i}-\alpha_{j}$,

$$
\boldsymbol{U}_{1, \boldsymbol{u}}:=\left[\begin{array}{cccccccccc}
2 \alpha_{1} & 0 & 0 & 0 & 0 & 2 \alpha_{3,2} & 4 \alpha_{2} & 0 & 0 & 0 \\
2 \alpha_{1} & 0 & 0 & 2 \alpha_{2,3} & 0 & 0 & 4 \alpha_{3} & 0 & 0 & 0 \\
0 & 2 \alpha_{2} & 0 & 2 \alpha_{1,3} & 0 & 0 & 0 & 4 \alpha_{3} & 0 & 0 \\
0 & 2 \alpha_{2} & 0 & 0 & 2 \alpha_{3,1} & 0 & 0 & 4 \alpha_{1} & 0 & 0 \\
0 & 0 & 2 \alpha_{3} & 0 & 2 \alpha_{2,1} & 0 & 0 & 0 & 4 \alpha_{1} & 0 \\
0 & 0 & 2 \alpha_{3} & 0 & 0 & 2 \alpha_{1,2} & 0 & 0 & 4 \alpha_{2} & 0 \\
0 & 0 & 0 & 0 & 0 & 2 \alpha_{3,2} & 4 \alpha_{1,3} & 0 & 0 & -6 \alpha_{1} \\
0 & 0 & 0 & 2 \alpha_{2,3} & 0 & 0 & 4 \alpha_{1,2} & 0 & 0 & -6 \alpha_{1} \\
0 & 0 & 0 & 2 \alpha_{1,3} & 0 & 0 & 0 & 4 \alpha_{2,1} & 0 & -6 \alpha_{2} \\
0 & 0 & 0 & 0 & 2 \alpha_{3,1} & 0 & 0 & 4 \alpha_{2,3} & 0 & -6 \alpha_{2} \\
0 & 0 & 0 & 0 & 2 \alpha_{2,1} & 0 & 0 & 0 & 4 \alpha_{3,2} & -6 \alpha_{3} \\
0 & 0 & 0 & 0 & 0 & 2 \alpha_{1,2} & 0 & 0 & 4 \alpha_{3,1} & -6 \alpha_{3}
\end{array}\right]
$$

$$
\boldsymbol{U}_{2, \boldsymbol{u}}=\left[\begin{array}{cccccccccccc}
2 \alpha_{1} & 2 \alpha_{2} & 0 & 0 & 0 & 0 & 0 & 0 & 0 & 0 & 0 & 2 \alpha_{3} \\
0 & 0 & 0 & 2 \alpha_{1} & 2 \alpha_{2} & 2 \alpha_{3} & 0 & 0 & 0 & 0 & 0 & 0 \\
0 & 0 & 0 & 0 & 0 & 0 & 0 & 2 \alpha_{2} & 2 \alpha_{3} & 2 \alpha_{1} & 0 & 0 \\
0 & \alpha_{1,3} & 3 \alpha_{3} & \alpha_{2,3} & 0 & 0 & 0 & 0 & 0 & 0 & 0 & 0 \\
0 & 0 & 0 & 0 & 0 & \alpha_{2,1} & 3 \alpha_{1} & \alpha_{3,1} & 0 & 0 & 0 & 0 \\
0 & 0 & 0 & 0 & 0 & 0 & 0 & 0 & 0 & \alpha_{3,2} & 3 \alpha_{2} & \alpha_{1,2} \\
0 & \frac{\alpha_{1,3}}{2} & \frac{3 \alpha_{2}}{2} & 0 & 0 & 0 & 0 & 0 & 0 & 0 & \frac{3 \alpha_{3}}{2} & \frac{\alpha_{1}, 2}{2} \\
0 & 0 & \frac{3 \alpha_{1}}{2} & \frac{\alpha_{2,3}}{2} & 0 & \frac{\alpha_{2,1}}{2} & \frac{3 \alpha_{3}}{2} & 0 & 0 & 0 & 0 & 0 \\
0 & 0 & 0 & 0 & 0 & 0 & \frac{3 \alpha_{2}}{2} & \frac{\alpha_{3,1}}{2} & 0 & \frac{\alpha_{3,2}}{2} & \frac{3 \alpha_{1}}{2} & 0 \\
0 & 0 & -2 \alpha_{3} & 0 & 0 & 0 & -2 \alpha_{1} & 0 & 0 & 0 & -2 \alpha_{2} & 0
\end{array}\right],
$$

and

$$
\boldsymbol{U}_{1, \boldsymbol{u}}^{k}=\boldsymbol{U}_{1, \boldsymbol{u}}\left(k, \boldsymbol{g}_{1}^{k}\right), \quad \boldsymbol{U}_{2, \boldsymbol{u}}^{k}=\boldsymbol{U}_{2, \boldsymbol{u}}\left(\boldsymbol{g}_{1}^{k}, \boldsymbol{g}_{2}^{k}\right) .
$$

For a spline $f_{d}:=\boldsymbol{s}_{d}^{T} \boldsymbol{c}=\sum_{j=1}^{n_{d}} c_{j} S_{j, d}$

$$
D_{u} f_{d}=d \boldsymbol{s}_{d-1}^{T} \boldsymbol{c}^{[1]}=d \sum_{j=1}^{n_{d-1}} \boldsymbol{c}_{j}^{[1]} S_{j, d-1}
$$


TABLE 2. The coefficients $c_{j}^{[1]}$ in (3.25). Here $\alpha_{i, j}:=\alpha_{i}-\alpha_{j}$, $c_{i, j}:=c_{i}-c_{j}$.

\begin{tabular}{|c|c|c|}
\hline$j$ & $c_{j}^{[1]}, d=1$ & $c_{j}^{[1]}, d=2$ \\
\hline 1 & $2 \alpha_{3,2} c_{6,1}+4 \alpha_{2} c_{7,1}$ & $2 \alpha_{2} c_{2,1}+2 \alpha_{3} c_{12,1}$ \\
\hline 2 & $4 \alpha_{3} c_{7,1}+2 \alpha_{2,3} c_{4,1}$ & $2 \alpha_{1} c_{4,5}+2 \alpha_{3} c_{6,5}$ \\
\hline 3 & $2 \alpha_{1,3} c_{4,2}+4 \alpha_{3} c_{8,2}$ & $2 \alpha_{2} c_{8,9}+2 \alpha_{1} c_{10,9}$ \\
\hline 4 & $4 \alpha_{1} c_{8,2}+2 \alpha_{3,1} c_{5,2}$ & $\alpha_{1,3} c_{2,3}+\alpha_{2,3} c_{4,3}$ \\
\hline 5 & $2 \alpha_{2,1} c_{5,3}+4 \alpha_{1} c_{9,3}$ & $\alpha_{2,1} c_{6,7}+\alpha_{3,1} c_{8,7}$ \\
\hline 6 & $4 \alpha_{2} c_{9,3}+2 \alpha_{1,2} c_{6,3}$ & $\alpha_{3,2} c_{10,11}+\alpha_{1,2} c_{12,11}$ \\
\hline 7 & $2 \alpha_{3,2} c_{6,10}+4 \alpha_{1,3} c_{7,10}$ & $1 / 2 \alpha_{1,3} c_{2,3}+\alpha_{2,3} c_{3,11}+1 / 2 \alpha_{1,2} c_{12,11}$ \\
\hline 8 & $4 \alpha_{1,2} c_{7,10}+2 \alpha_{2,3} c_{4,10}$ & $1 / 2 \alpha_{2,3} c_{4,3}+1 / 2 \alpha_{2,1} c_{6,7}+\alpha_{3,1} c_{7,3}$ \\
\hline 9 & $2 \alpha_{1,3} c_{4,10}+4 \alpha_{2,1} c_{8,10}$ & $1 / 2 \alpha_{3,1} c_{8,7}+1 / 2 \alpha_{3,2} c_{10,11}+\alpha_{1,2} c_{11,7}$ \\
\hline 10 & $4 \alpha_{2,3} c_{8,10}+2 \alpha_{3,1} c_{5,10}$ & $2 / 3 \alpha_{3,1} c_{7,3}+2 / 3 \alpha_{2,3} c_{3,11}+2 / 3 \alpha_{1,2} c_{11,7}$ \\
\hline 11 & $2 \alpha_{2,1} c_{5,10}+4 \alpha_{3,2} c_{9,10}$ & \\
\hline 12 & $4 \alpha_{3,1} c_{9,10}+2 \alpha_{1,2} c_{6,10}$ & \\
\hline
\end{tabular}

where the $c_{j}^{[1]}$ are given in Table 2, Moreover, for $k=1, \ldots, 12$

$$
D_{\boldsymbol{u}} \sum_{j \in \mathcal{G}_{d}^{k}} c_{j} S_{j, d}^{k}=d \sum_{j \in \mathcal{G}_{d-1}^{k}} c_{j}^{[1]} S_{j, d-1}^{k}, \quad d=1,2 .
$$

Proof. Let $1 \leq k \leq 12$ and $\boldsymbol{x}, \boldsymbol{y} \in \mathbb{R}^{2}$. Recall from (3.13) that

$$
\boldsymbol{R}_{d}^{k}(\boldsymbol{x}) \boldsymbol{\psi}_{d}^{k}(\boldsymbol{y})=\left(1-\boldsymbol{x}^{T} \boldsymbol{y}\right) \boldsymbol{\psi}_{d-1}^{k}(\boldsymbol{y}), \quad \boldsymbol{x}=\left[\begin{array}{ll}
x_{1} & x_{2}
\end{array}\right]^{T}, \boldsymbol{y}=\left[\begin{array}{ll}
y_{1} & y_{2}
\end{array}\right]^{T} \in \mathbb{R}^{2} .
$$

Let $r \in\{1,2\}$. Differentiation gives

$$
\frac{\partial}{\partial x_{r}} \boldsymbol{R}_{d}^{k}(\boldsymbol{x}) \boldsymbol{\psi}_{d}^{k}(\boldsymbol{y})=-y_{r} \boldsymbol{\psi}_{d-1}^{k}(\boldsymbol{y})
$$

But, then

$$
\begin{aligned}
\frac{\partial}{\partial x_{r}} \boldsymbol{s}_{d}^{k}(\boldsymbol{x})^{T} \boldsymbol{\psi}_{d}^{k}(\boldsymbol{y}) \stackrel{\stackrel{(3.5)}{=}}{=} \frac{\partial}{\partial x_{r}}\left(1-\boldsymbol{x}^{T} \boldsymbol{y}\right)^{d}=-d y_{r}\left(1-\boldsymbol{x}^{T} \boldsymbol{y}\right)^{d-1} \\
\stackrel{(3.5)}{=}-d y_{r} \boldsymbol{s}_{d-1}^{k}(\boldsymbol{x})^{T} \boldsymbol{\psi}_{d-1}^{k}(\boldsymbol{y}) \\
\stackrel{\sqrt[3.27]{=}}{=} d \boldsymbol{s}_{d-1}^{k}(\boldsymbol{x})^{T} \frac{\partial}{\partial x_{r}} \boldsymbol{R}_{d}^{k}(\boldsymbol{x}) \boldsymbol{\psi}_{d}^{k}(\boldsymbol{y}) .
\end{aligned}
$$

Since the elements of $\boldsymbol{\psi}_{d}^{k}$ are linearly independent it follows that

$$
\frac{\partial}{\partial x_{r}} \boldsymbol{s}_{d}^{k}(\boldsymbol{x})^{T}=d \boldsymbol{s}_{d-1}^{k}(\boldsymbol{x})^{T} \frac{\partial}{\partial x_{r}} \boldsymbol{R}_{d}^{k}(\boldsymbol{x}), \quad r=1,2
$$

and (3.21) with $\boldsymbol{U}_{d, \boldsymbol{u}}^{k}=D_{\boldsymbol{u}} \boldsymbol{R}_{d}^{k}(\boldsymbol{x})$ follows by linearity of the directional derivative. Moreover, (3.20), with $\boldsymbol{U}_{d, \boldsymbol{u}}=D_{\boldsymbol{u}} \boldsymbol{R}_{d}(\boldsymbol{x})$, follows immediately from (3.21).

Let $\boldsymbol{x}$ have barycentric coordinates $\boldsymbol{\beta}$ with respect to $\Delta$. For $t \in \mathbb{R}$ the barycentric coordinates of $\boldsymbol{x}+t \boldsymbol{u}$ are $\boldsymbol{\beta}+t \boldsymbol{\alpha}$, where the $\boldsymbol{\alpha}$ is determined from (3.19). But 
then,

$$
\begin{aligned}
\boldsymbol{U}_{d, \boldsymbol{u}}(\boldsymbol{x})=D_{\boldsymbol{u}} \boldsymbol{R}_{d}(\boldsymbol{x}) & =\left.\frac{d}{d t} \boldsymbol{R}_{d}\left(\left(\beta_{1}+t \alpha_{1}\right) \boldsymbol{p}_{1}+\left(\beta_{2}+t \alpha_{2}\right) \boldsymbol{p}_{2}+\left(\beta_{3}+t \alpha_{3}\right) \boldsymbol{p}_{3}\right)\right|_{t=0} \\
& =\alpha_{1} \frac{\partial \boldsymbol{R}_{d}}{\partial \beta_{1}}+\alpha_{2} \frac{\partial \boldsymbol{R}_{d}}{\partial \beta_{2}}+\alpha_{3} \frac{\partial \boldsymbol{R}_{d}}{\partial \beta_{3}} \\
& =: \boldsymbol{\alpha}^{T} \nabla_{\beta} \boldsymbol{R}_{d} .
\end{aligned}
$$

$\boldsymbol{R}_{d}$ has elements like $\gamma_{j}=2 \beta_{j}-1$ and $\beta_{i, j}=\beta_{i}-\beta_{j}$. Therefore,

$$
\frac{\partial \gamma_{j}}{\partial \beta_{k}}=2 \delta_{k, j} \quad \text { and } \quad \frac{\partial \beta_{i, j}}{\partial \beta_{k}}=\delta_{k, i}-\delta_{k, j},
$$

and the formulas for $\boldsymbol{U}_{1, \boldsymbol{u}}$ and $\boldsymbol{U}_{2, \boldsymbol{u}}$ follow.

To prove (3.25) we note that (3.20) implies that $\boldsymbol{c}^{[1]}=\boldsymbol{U}_{d, \boldsymbol{u}} \boldsymbol{c}$. The entries in Table 2 are then obtained by eliminating one $\alpha_{j}$ using $\alpha_{1}+\alpha_{2}+\alpha_{3}=0$ and regrouping terms.

For $d=k=1$ (3.26) takes the form $D_{\boldsymbol{u}}\left(c_{1} S_{1,1}+c_{6} S_{6,1}+c_{7} S_{7,1}\right)=2 \alpha_{3,2} c_{6,1}+$ $4 \alpha_{2} c_{7,1}$ or in terms of control points $D_{\boldsymbol{u}}\left(\boldsymbol{c}_{1} S_{1,1}+\boldsymbol{c}_{6} S_{6,1}+\boldsymbol{c}_{7} S_{7,1}\right)=2 \alpha_{3,2} \boldsymbol{c}_{6,1}+$ $4 \alpha_{2} \boldsymbol{c}_{7,1}$, where $\boldsymbol{c}_{j}=\left(\boldsymbol{p}_{j}, c_{j}\right)$ and $\boldsymbol{c}_{i, j}=\boldsymbol{c}_{i}-\boldsymbol{c}_{j}$. This involves two differences of control points pointing from $\boldsymbol{c}_{1}$ to $\boldsymbol{c}_{6}$ and from $\boldsymbol{c}_{1}$ to $\boldsymbol{c}_{7}$. Similarly, on an inner triangle, say $k=7$, (3.26) will involve differences of control points pointing from $\boldsymbol{c}_{10}$ to control points $\boldsymbol{c}_{6}$ and $\boldsymbol{c}_{7}$ of $\Delta_{7}$. Only those two types of differences are involved in computing $\boldsymbol{c}^{[1]}$. For $d=2$, to each component $c_{i}^{[1]}$ of $\boldsymbol{c}^{[1]}$ there is a corresponding triangular or quadrilateral region. The differences of control points used are along directed edges of that region.

There is also a simple differentiation formula for the second derivative of a quadratic S-spline.

Theorem 3.10. For direction vectors $\boldsymbol{u}, \boldsymbol{v} \in \mathbb{R}^{2}$ and any $\boldsymbol{x}$ not on a knot line of $\Delta$, we have

$$
\begin{aligned}
& D_{\boldsymbol{v}} D_{\boldsymbol{u}} \boldsymbol{s}_{2}^{k}(\boldsymbol{x})^{T}=2 \boldsymbol{U}_{1, \boldsymbol{v}}^{k} \boldsymbol{U}_{2, \boldsymbol{u}}^{k}=2 \boldsymbol{U}_{1, \boldsymbol{u}}^{k} \boldsymbol{U}_{2, \boldsymbol{v}}^{k} \\
& D_{\boldsymbol{v}} D_{\boldsymbol{u}} \boldsymbol{s}_{2}(\boldsymbol{x})^{T}=2 \boldsymbol{s}_{0}(\boldsymbol{x})^{T} \boldsymbol{U}_{1, \boldsymbol{v}} \boldsymbol{U}_{2, \boldsymbol{u}}=2 \boldsymbol{s}_{0}(\boldsymbol{x})^{T} \boldsymbol{U}_{1, \boldsymbol{u}} \boldsymbol{U}_{2, \boldsymbol{v}}
\end{aligned}
$$

where the $\boldsymbol{U}$ matrices are defined according to the conventions in Theorem 3.9 .

Proof. Applying $D_{\boldsymbol{v}}$ to (3.21) and since $\boldsymbol{U}_{2, \boldsymbol{u}}^{k}:=D_{\boldsymbol{u}} \boldsymbol{R}_{2}^{k}(\boldsymbol{x})$ is independent of $\boldsymbol{x}$,

$$
D_{\boldsymbol{v}} D_{\boldsymbol{u}} \boldsymbol{s}_{2}^{k}(\boldsymbol{x})^{T}=2 D_{\boldsymbol{v}}\left(\boldsymbol{s}_{1}^{k}(\boldsymbol{x})^{T} D_{\boldsymbol{u}} \boldsymbol{R}_{2}^{k}\right)=2 D_{\boldsymbol{v}} \boldsymbol{R}_{1}^{k} D_{\boldsymbol{u}} \boldsymbol{R}_{2}^{k}=2 \boldsymbol{U}_{1, \boldsymbol{v}}^{k} \boldsymbol{U}_{2, \boldsymbol{u}}^{k} .
$$

The commutativity of differentiation follows from Lemma 3.8.

\section{Evaluation algorithms}

Consider computing an S-spline $f_{d}(\boldsymbol{x})=\boldsymbol{s}_{d}(\boldsymbol{x})^{T} \boldsymbol{c}$. If $\boldsymbol{x} \in \Delta_{k}$, then by Corollary 2.8 ,

$$
f_{d}(\boldsymbol{x})=\boldsymbol{s}_{d}^{k}(\boldsymbol{x}) \boldsymbol{c}^{0}=\prod_{i=1}^{d} \boldsymbol{R}_{i}^{k}(\boldsymbol{x}) \boldsymbol{c}^{0}, \quad \boldsymbol{c}^{0}:=\boldsymbol{c}\left(\boldsymbol{g}_{d}^{k}\right), \quad d=1,2 .
$$

We also consider computing directional derivatives $D_{\boldsymbol{u}} f_{d}$ and $D_{\boldsymbol{v}} D_{\boldsymbol{u}} f_{d}$ of order $r=1$ and $r=2$, respectively.

For $d=1$ and $\boldsymbol{x} \in \Delta_{k}$ we start with $\boldsymbol{c}^{0}=\boldsymbol{c}\left(\boldsymbol{g}_{1}^{k}\right)$. Then for $r=0, D_{\boldsymbol{u}}^{0} f_{1}(\boldsymbol{x})=$ $f_{1}(\boldsymbol{x})=\boldsymbol{R}_{1}^{k}(\boldsymbol{x}) \boldsymbol{c}^{0}$, while $D_{\boldsymbol{u}}^{1} f_{1}(\boldsymbol{x})=\boldsymbol{U}_{1, \boldsymbol{u}}^{k} \boldsymbol{c}^{0}$ for $r=1$. In the quadratic case, 


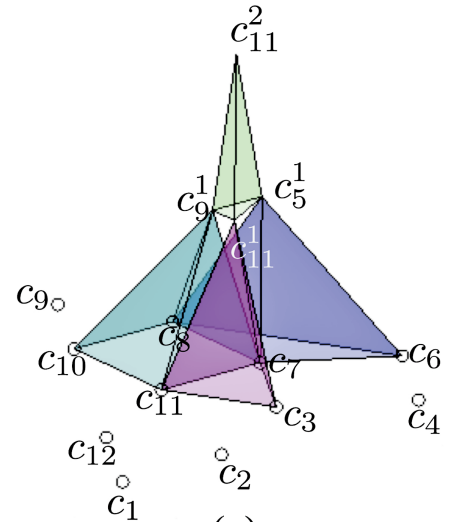

(a)

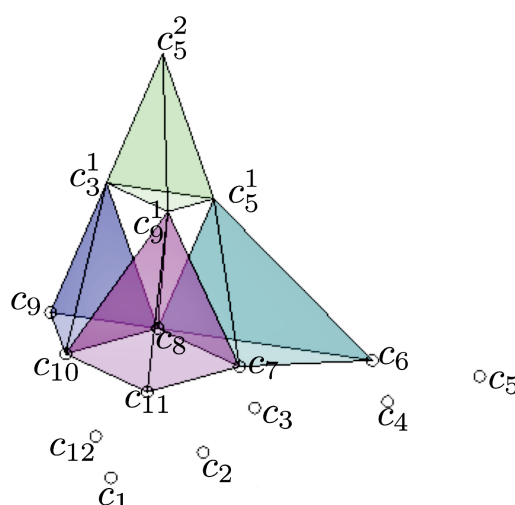

(b)

Figure 8. Evaluating $f_{2}(\boldsymbol{x})$ using Algorithm 4.1 for (a) $\boldsymbol{x} \in \Delta_{11}$, and (b) $\boldsymbol{x} \in \Delta_{5}$

$d=2$, and $\boldsymbol{x} \in \Delta_{k}$ we start with $\boldsymbol{c}^{0}=\boldsymbol{c}\left(\boldsymbol{g}_{2}^{k}\right)$. Then for $r=0, \boldsymbol{c}^{1}=\boldsymbol{R}_{2}^{k}(\boldsymbol{x}) \boldsymbol{c}^{0}$ and $D_{\boldsymbol{u}}^{0} f_{2}(\boldsymbol{x})=f_{2}(\boldsymbol{x})=\boldsymbol{R}_{1}^{k}(\boldsymbol{x}) \boldsymbol{c}^{1}$ and for $r=1, D_{\boldsymbol{u}}^{1} f_{2}(\boldsymbol{x})=\boldsymbol{c}^{2}=\boldsymbol{R}_{1}^{k}(\boldsymbol{x}) \boldsymbol{c}^{1}$, where $\boldsymbol{c}^{1}=2 \boldsymbol{U}_{2, \boldsymbol{u}}^{k} \boldsymbol{c}^{0}$. Finally for $r=2, D_{\boldsymbol{v}} D_{\boldsymbol{u}} f_{2}(\boldsymbol{x})=\boldsymbol{c}^{2}=\boldsymbol{U}_{1, \boldsymbol{v}}^{k} \boldsymbol{c}^{1}$, while $\boldsymbol{c}^{1}=2 \boldsymbol{U}_{2, \boldsymbol{u}}^{k} \boldsymbol{c}^{0}$.

We give two algorithms. Going from right to left, we obtain $D_{u}^{r} f_{d}(\boldsymbol{x})$, and, from left to right, we can compute the S-spline elements $D_{\boldsymbol{u}}^{r} \boldsymbol{s}_{d}^{k}(\boldsymbol{x})$ that can be nonzero at $\boldsymbol{x}$.

Algorithm 4.1. Given $\boldsymbol{x} \in \Delta, r \in\{0,1,2\}, \boldsymbol{u} \in \mathbb{R}^{2}$ if $r>0$, and coefficients $\boldsymbol{c}$.

(1) Determine the barycentric coordinates of $\boldsymbol{x}$ with respect to $\Delta$ and $k$ such that $\boldsymbol{x} \in \Delta_{k}$ using Algorithm 1.1.

(2) $\boldsymbol{c}^{0}=\boldsymbol{c}\left(\boldsymbol{g}_{d}^{k}\right)$.

(3) for $i=0, \ldots, r-1$

$\boldsymbol{c}^{i+1}=(d-i) \boldsymbol{U}_{d-i, \boldsymbol{u}}^{k} \boldsymbol{c}^{i}$.

(4) for $i=r, \ldots, d-1$

$\boldsymbol{c}^{i+1}=\boldsymbol{R}_{d-i}^{k}(\boldsymbol{x}) \boldsymbol{c}^{i}$

(5) $D_{\boldsymbol{u}}^{r} f_{d}(\boldsymbol{x})=\boldsymbol{c}^{d}$.

In Figure 8, the algorithm is illustrated for $d=2, r=0$, and 2 values of $k$. Table 1 gives the relevant values of $\boldsymbol{g}_{1}^{k}$ and $\boldsymbol{g}_{2}^{k}$. For $k=5$, the 6 coefficients with indices $6,7,8,9,10,11$ are combined to give $c_{j}^{1}, j=3,5,9$, and these 3 , in turn, give rise to $f_{2}(\boldsymbol{x})=c_{5}^{2}$. The $c_{j}$ 's needed for $\boldsymbol{x} \in \Delta_{11}$ have indices $3,6,7,8,10,11$ giving $c_{j}^{1}, j=5,9,11$, and these 3 give rise to $f_{2}(\boldsymbol{x})=c_{11}^{2}$. We see that $c_{9}^{1}$ requires $4 c_{j}$ 's, while the other two level one coefficients combine only $3 c_{j}$ 's.

Consider next an algorithm for computing $D_{\boldsymbol{u}}^{r} \boldsymbol{s}_{d}^{k}(\boldsymbol{x})^{T}$ for some $\boldsymbol{x} \in \Delta$. If $\boldsymbol{x} \in \Delta_{k}$, then $\boldsymbol{s}_{d}^{k}(\boldsymbol{x})^{T}=\prod_{i=1}^{d} \boldsymbol{R}_{i}^{k}(\boldsymbol{x})$, and this leads to,

Algorithm 4.2. Given $\boldsymbol{x} \in \Delta, r \in\{0,1,2\}$, and $\boldsymbol{u} \in \mathbb{R}^{2}$ if $r>0$.

(1) Determine the barycentric coordinates of $\boldsymbol{x}$ with respect to $\Delta$ and $k$ such that $\boldsymbol{x} \in \Delta_{k}$ using Algorithm 1.1.

(2) $\boldsymbol{s}_{0}^{k}=1$.

(3) for $i=1, \ldots, d-r$ $\boldsymbol{s}_{i}^{k}=\boldsymbol{s}_{i-1}^{k} \boldsymbol{R}_{i}^{k}(\boldsymbol{x})$. 
(4) for $i=d-r+1, \ldots, d$

$$
\boldsymbol{s}_{i}^{k}=i \boldsymbol{s}_{i-1}^{k} \boldsymbol{U}_{i, \boldsymbol{u}}^{k} \text {. }
$$

(5) $D_{u}^{r} s_{d}^{k}(\boldsymbol{x})^{T}=\boldsymbol{s}_{d}^{k}$

Since the elements in $\boldsymbol{R}_{1}^{k}(\boldsymbol{x})$ and $\boldsymbol{R}_{2}^{k}(\boldsymbol{x})$ are nonnegative for $\boldsymbol{x} \in \Delta_{k}$ the algorithms for $r=0$ are quite stable.

\section{Subdivision}

Suppose we divide the triangle $\Delta$ in Figure 2 uniformly into 4 triangles $\delta_{1}, \delta_{2}, \delta_{3}$, $\delta_{4}$, and on each of these triangles we use the PS12-split. Thus, we develop a triangulation $\Delta_{P S 12}^{(1)}$ of $\Delta$ comprised of 48 triangles (Figure 9). On $\delta_{i}$ we have $n_{d}$ linearly independent S-splines $W_{n_{d}(i-1)+j, d}, j=1, \ldots, n_{d}$ for $i=1, \ldots, 4$, for a total of,

$$
I_{d}:=4 n_{d}
$$

Since $\mathbb{S}_{d}\left(\Delta_{P S 12}\right) \subset \mathbb{S}_{d}\left(\Delta_{P S 12}^{(1)}\right)$ and $\boldsymbol{W}_{d}$ spans $\mathbb{S}_{d}\left(\Delta_{P S 12}^{(1)}\right)$, the S-spline $S_{j, d}$ is a linear combination of the $W_{j, d}$,

$$
S_{j, d}=\sum_{i=1}^{I_{d}} \alpha_{j, d}(i) W_{i, d}, \quad j=1, \ldots, n_{d} .
$$

In analogy to B-splines, the numbers $\alpha_{j, d}(i)$ are discrete S-splines of degree $d$ relating $\Delta_{P S 12}$ to $\Delta_{P S 12}^{(1)}$, and the matrix,

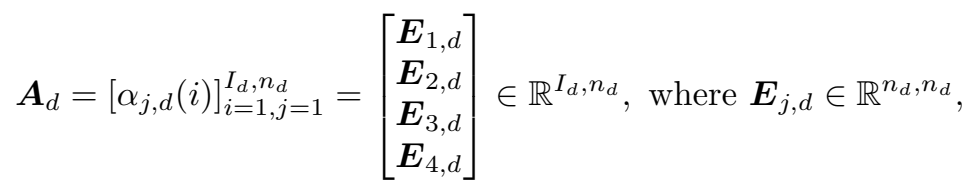

is called the knot insertion matrix of degree $d$ taking $\Delta_{P S 12}$ to $\Delta_{P S 12}^{(1)}$.

In succinct vector form (5.1) is expressed by,

$$
\boldsymbol{s}_{d}^{T}=\boldsymbol{w}_{d}^{T} \boldsymbol{A}_{d},
$$

where

$$
\boldsymbol{s}_{d}^{T}:=\left[S_{1, d}, \ldots, S_{n_{d}, d}\right], \quad \boldsymbol{w}_{d}^{T}:=\left[W_{1, d}, \ldots, W_{I_{d}, d}\right]
$$

If

$$
f=\sum_{j=1}^{n_{d}} c_{j} S_{j, d}=\boldsymbol{s}_{d}^{T} \boldsymbol{c}=\boldsymbol{w}_{d}^{T} \boldsymbol{\omega}=\sum_{i=1}^{I_{d}} \omega_{i} W_{i, d}
$$

then

$$
\boldsymbol{\omega}=\boldsymbol{A}_{d} \boldsymbol{c}
$$

To compute the coefficients of the subdivided surface we can either: i) compute the knot insertion matrix and do a matrix vector multiplication or use a modified approach that directly averages the original coefficients. We will present both and start by deriving the knot insertion matrix. 


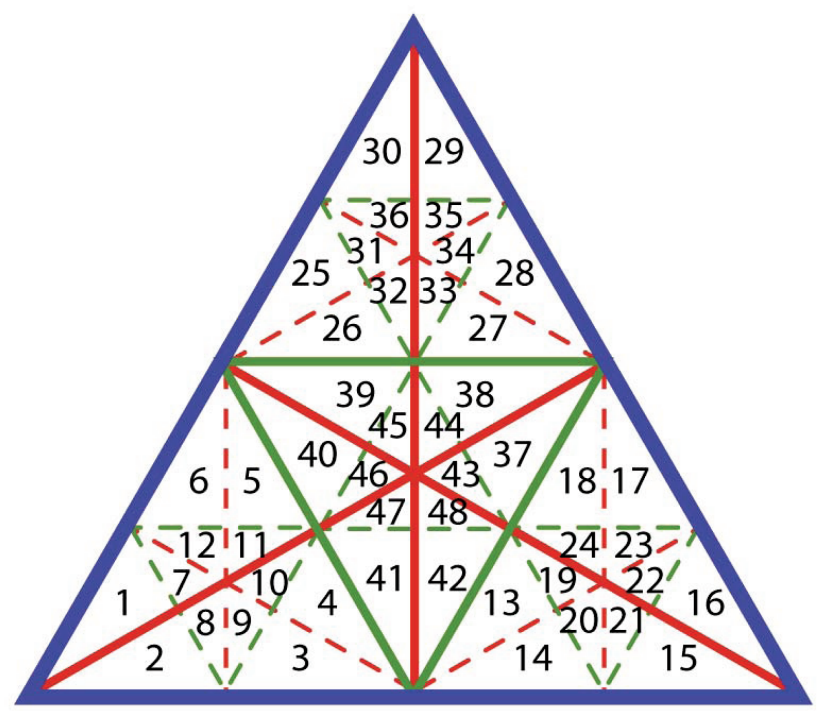

Figure 9. A triangle divided into 48 triangles

5.1. Computing the knot insertion matrix. To determine the matrix $\boldsymbol{A}_{d}$ we start with a lemma involving the dual polynomials $\psi_{j, d}$ of $S_{j, d}$ and $\phi_{i, d}$ of $W_{i, d}$. For fixed $i$ with $1 \leq i \leq I_{d}$ we let $\boldsymbol{x}_{1}, \ldots, \boldsymbol{x}_{d}$ be the dual points of $W_{i, d}$, so that,

$$
\phi_{i, d}(\boldsymbol{y})=\prod_{r=1}^{d}\left(1-\boldsymbol{x}_{r}^{T} \boldsymbol{y}\right) .
$$

Recall that the 12 triangles of the PS12-split of $\Delta$ are denoted by $\Delta_{k}$, for $k=$ $1, \ldots, 12$. It is easy to see that the $d$ dual points of $W_{i, d}$ are located in (at least) one closed triangle $\bar{\Delta}_{k_{i}}$, the closure of $\Delta_{k_{i}}$.

Lemma 5.1. For fixed $i, 1 \leq i \leq I_{d}$ and $d=1,2$, we get

$$
\phi_{i, d}=\sum_{j=1}^{n_{d}} \alpha_{j, d}(i) \psi_{j, d}=\sum_{j \in \mathcal{L}_{i, d}} \alpha_{j, d}(i) \psi_{j, d}
$$

and

$$
\left\{j: \alpha_{j, d}(i) \neq 0\right\} \subset \mathcal{L}_{i, d},
$$

where

$$
\mathcal{L}_{i, d}:=\left\{j: \operatorname{supp}\left(W_{i, d}\right) \subset \operatorname{supp}\left(S_{j, d}\right)\right\} .
$$

Moreover, $\mathcal{L}_{i, d} \subset \mathcal{G}_{d}^{k}$ for all $k$ such that $\Delta_{k} \cap \operatorname{supp}\left(W_{i, d}\right) \neq \emptyset$. In particular, the number of elements in $\mathcal{L}_{i, d}$ is at most $\nu_{d}$.

Proof. Fix $i$. By linear independence of the $W_{i, d}$ it follows that $\alpha_{j, d}(i) \neq 0$ only if the support of $W_{i, d}$ is contained in the support of $S_{j, d}$. Therefore, if $\alpha_{j, d}(i) \neq 0$, 
then $j \in \mathcal{L}_{i, d}$. Using (3.4) and (5.1), for $\boldsymbol{x} \in \Delta$ and any $\boldsymbol{y} \in \mathbb{R}$,

$$
\begin{aligned}
\left(1-\boldsymbol{x}^{T} \boldsymbol{y}\right)^{d} & \stackrel{\sqrt{3.4}}{=} \sum_{j=1}^{n_{d}} S_{j, d}(\boldsymbol{x}) \psi_{j, d}(\boldsymbol{y}) \\
& \stackrel{\sqrt{5.1}}{=} \sum_{j=1}^{n_{d}}\left(\sum_{i=1}^{I_{d}} \alpha_{j, d}(i) W_{i, d}(\boldsymbol{x})\right) \psi_{j, d}(\boldsymbol{y}) \\
& =\sum_{i=1}^{I_{d}}\left(\sum_{j=1}^{n_{d}} \alpha_{j, d}(i) \psi_{j, d}(\boldsymbol{y})\right) W_{i, d}(\boldsymbol{x}) .
\end{aligned}
$$

On the other hand, by adding together the Marsden like identities on $\delta_{r}$ for $r=$ $1, \ldots, 4$, we also have,

$$
\left(1-\boldsymbol{x}^{T} \boldsymbol{y}\right)^{d}=\sum_{i=1}^{I_{d}} \phi_{i, d}(\boldsymbol{y}) W_{i, d}(\boldsymbol{x}) .
$$

Equation (5.6) follows from the linear independence of the $I_{d}$ S-splines $W_{i, d}$ on $\Delta$ and (5.7).

Suppose $\Delta_{k} \cap \operatorname{supp}\left(W_{i, d}\right) \neq \emptyset$. Then, for all $j \in \mathcal{L}_{i, d}, \Delta_{k} \cap \operatorname{supp}\left(S_{j, d}\right) \neq \emptyset$, and simplex spline properties assure $\Delta_{k} \subset \operatorname{supp}\left(S_{j, d}\right)$. Therefore, $j \in \mathcal{G}_{d}^{k}$, so $\mathcal{L}_{i, d} \subset \mathcal{G}_{d}^{k}$. Noting $\mathcal{G}_{d}^{k}$ has exactly $\nu_{d}$ elements independent of $k$, the result follows.

A recurrence relation for the $\alpha_{j, d}$ 's in the $i$ th row of $\boldsymbol{A}_{d}$ can be determined from the following theorem.

Theorem 5.2. Suppose, for fixed $d \in\{1,2\}$ and $1 \leq i \leq I_{d}$, that $\boldsymbol{x}_{1}, \ldots, \boldsymbol{x}_{d}$ are the dual points of $W_{i, d}$. Also, let $k$ be such that $\boldsymbol{x}_{1}, \ldots, \boldsymbol{x}_{d} \in \bar{\Delta}_{k}$. Then

$$
\boldsymbol{\alpha}_{d}^{k}(i)^{T}=\boldsymbol{A}_{d}\left(i, \boldsymbol{g}_{d}^{k}\right)=\prod_{r=1}^{d} \boldsymbol{R}_{r}^{k}\left(\boldsymbol{x}_{r}\right)
$$

and

$$
\boldsymbol{\alpha}_{d}(i)^{T}=\left[\alpha_{1, d}(i), \ldots, \alpha_{n_{d}, d}(i)\right]=\boldsymbol{e}_{k}^{T} \prod_{r=1}^{d} \boldsymbol{R}_{r}\left(\boldsymbol{x}_{r}\right) .
$$

Moreover, $\alpha_{j, d}(i)=0$ for $j \notin \mathcal{G}_{d}^{k}$. Here $\boldsymbol{R}_{1}(\boldsymbol{x})$ is given by (2.15); $\boldsymbol{R}_{2}(\boldsymbol{x})$, by (2.16); and $\boldsymbol{R}_{d}^{k}$, in Definition 2.7.

Proof. By Lemma 5.1

$$
\phi_{i, d}(\boldsymbol{y})=\sum_{j \in \mathcal{G}_{d}^{k}} \alpha_{j, d}(i) \psi_{j, d}(\boldsymbol{y})=\boldsymbol{\alpha}_{d}^{k}(i)^{T} \boldsymbol{\psi}_{d}^{k}(y) .
$$

By 3.16

$$
\phi_{i, d}(\boldsymbol{y})=\prod_{r=1}^{d}\left(1-\boldsymbol{x}_{r}^{T} \boldsymbol{y}\right)=\prod_{r=1}^{d} \boldsymbol{R}_{r}^{k}\left(\boldsymbol{x}_{r}\right) \boldsymbol{\psi}_{d}^{k}(\boldsymbol{y})
$$


Theorem 3.6 shows that the set given by the vector $\boldsymbol{\psi}_{d}^{k}$ is linearly independent, so (5.9) follows. From (5.7) the remaining $\alpha_{j, d}$ 's in row $i$ of $\boldsymbol{A}_{d}$ are 0. Thus, (5.10) follows (cf. Lemma 5.1).

The blocks $\boldsymbol{E}_{r, 2}$ of the quadratic subdivision matrix (5.2) are as follows:

$$
\boldsymbol{E}_{1,2}=\left[\begin{array}{cccccccccccc}
1 & 0 & 0 & 0 & 0 & 0 & 0 & 0 & 0 & 0 & 0 & 0 \\
\frac{1}{2} & \frac{1}{2} & 0 & 0 & 0 & 0 & 0 & 0 & 0 & 0 & 0 & 0 \\
\frac{1}{6} & \frac{5}{12} & \frac{1}{4} & 0 & 0 & 0 & 0 & 0 & 0 & 0 & 0 & \frac{1}{6} \\
0 & \frac{3}{4} & 0 & \frac{1}{4} & 0 & 0 & 0 & 0 & 0 & 0 & 0 & 0 \\
0 & \frac{1}{2} & 0 & \frac{1}{2} & 0 & 0 & 0 & 0 & 0 & 0 & 0 & 0 \\
0 & \frac{1}{4} & \frac{3}{4} & 0 & 0 & 0 & 0 & 0 & 0 & 0 & 0 & 0 \\
0 & \frac{1}{4} & \frac{1}{4} & 0 & 0 & 0 & 0 & 0 & 0 & 0 & \frac{1}{4} & \frac{1}{4} \\
0 & 0 & 0 & 0 & 0 & 0 & 0 & 0 & 0 & 0 & \frac{3}{4} & \frac{1}{4} \\
0 & 0 & 0 & 0 & 0 & 0 & 0 & 0 & 0 & \frac{1}{2} & 0 & \frac{1}{2} \\
0 & 0 & 0 & 0 & 0 & 0 & 0 & 0 & 0 & \frac{1}{4} & 0 & \frac{3}{4} \\
\frac{1}{6} & \frac{1}{6} & 0 & 0 & 0 & 0 & 0 & 0 & 0 & 0 & \frac{1}{4} & \frac{5}{12} \\
\frac{1}{2} & 0 & 0 & 0 & 0 & 0 & 0 & 0 & 0 & 0 & 0 & \frac{1}{2}
\end{array}\right],
$$

and

$$
\boldsymbol{E}_{4,2}=\left[\begin{array}{cccccccccccc}
0 & 0 & 0 & 0 & 0 & \frac{1}{2} & 0 & \frac{1}{2} & 0 & 0 & 0 & 0 \\
0 & 0 & 0 & 0 & 0 & 0 & \frac{3}{4} & \frac{1}{4} & 0 & 0 & 0 & 0 \\
0 & 0 & 0 & 0 & 0 & 0 & \frac{1}{2} & 0 & 0 & 0 & \frac{1}{2} & 0 \\
0 & 0 & 0 & 0 & 0 & 0 & 0 & 0 & 0 & \frac{1}{4} & \frac{3}{4} & 0 \\
0 & 0 & 0 & 0 & 0 & 0 & 0 & 0 & 0 & \frac{1}{2} & 0 & \frac{1}{2} \\
0 & 0 & 0 & 0 & 0 & 0 & 0 & 0 & 0 & 0 & \frac{3}{4} & \frac{1}{4} \\
0 & 0 & \frac{1}{2} & 0 & 0 & 0 & 0 & 0 & 0 & 0 & \frac{1}{2} & 0 \\
0 & \frac{1}{4} & \frac{3}{4} & 0 & 0 & 0 & 0 & 0 & 0 & 0 & 0 & 0 \\
0 & \frac{1}{2} & 0 & \frac{1}{2} & 0 & 0 & 0 & 0 & 0 & 0 & 0 & 0 \\
0 & 0 & \frac{3}{4} & \frac{1}{4} & 0 & 0 & 0 & 0 & 0 & 0 & 0 & 0 \\
0 & 0 & \frac{1}{2} & 0 & 0 & 0 & \frac{1}{2} & 0 & 0 & 0 & 0 & 0 \\
0 & 0 & 0 & 0 & 0 & \frac{1}{4} & \frac{3}{4} & 0 & 0 & 0 & 0 & 0
\end{array}\right]
$$

5.2. Coefficient averaging algorithm. For any $n \in \mathbb{N}$ and real numbers $r_{1}, \ldots, r_{n}$ we define,

$$
\mu\left(r_{1}, \ldots, r_{n}\right):=\sum_{j=1}^{n} r_{j} / n .
$$

As shorthand we write for some $\left[c_{1}, \ldots, c_{12}\right]^{T}$ and $1 \leq i_{1}<\cdots<i_{m} \leq 12$,

$$
\mu_{i_{1}, i_{2}, \ldots, i_{m}}=\mu\left(c_{i_{1}}, c_{i_{2}}, \ldots, c_{i_{m}}\right) .
$$

Continuing as defined above, we state the following. 
Theorem 5.3. If

$$
f=\sum_{j=1}^{12} c_{j} S_{j, 2}=\sum_{i=1}^{48} \omega_{i} W_{i, 2}
$$

then

$$
\omega_{12(r-1)+j}=\xi_{j, r}, \quad r=1,2,3,4, \quad j=1, \ldots, 12,
$$

where $\boldsymbol{\Xi}=\left[\xi_{j, r}\right] \in \mathbb{R}^{12,4}$ is the following:

$(5.14) \boldsymbol{\Xi}=\left[\begin{array}{cccc}c_{1} & \mu_{2,4} & \mu_{10,12} & \mu_{6,8} \\ \mu_{1,2} & \mu\left(\mu_{2,4}, c_{4}\right) & \mu\left(\mu_{10,11}, c_{11}\right) & \mu\left(\mu_{7,8}, c_{7}\right) \\ \mu\left(\mu_{1,2,12}, \mu_{2,3}\right) & \mu\left(\mu_{4,5,6}, \mu_{3,4}\right) & \mu\left(\mu_{7,8}, \mu_{10,11}\right) & \mu_{7,11} \\ \mu\left(c_{2}, \mu_{2,4}\right) & \mu_{4,5} & \mu\left(\mu_{7,8}, c_{7}\right) & \mu\left(\mu_{10,11}, c_{11}\right) \\ \mu_{2,4} & c_{5} & \mu_{6,8} & \mu_{10,12} \\ \mu\left(\mu_{2,3}, c_{3}\right) & \mu_{5,6} & \mu\left(\mu_{6,8}, c_{8}\right) & \mu\left(\mu_{11,12}, c_{11}\right) \\ \mu\left(\mu_{2,3}, \mu_{11,12}\right) & \mu\left(\mu_{4,5,6}, \mu_{6,7}\right) & \mu\left(\mu_{8,9,10}, \mu_{7,8}\right) & \mu_{3,11} \\ \mu\left(c_{11}, \mu_{11,12}\right) & \mu\left(c_{6}, \mu_{6,8}\right) & \mu_{8,9} & \mu\left(c_{3}, \mu_{2,3}\right) \\ \mu_{10,12} & \mu_{6,8} & c_{9} & \mu_{2,4} \\ \mu\left(\mu_{10,12}, c_{12}\right) & \mu\left(\mu_{6,7}, c_{7}\right) & \mu_{9,10} & \mu\left(\mu_{3,4}, c_{3}\right) \\ \mu\left(\mu_{1,2,12}, \mu_{11,12}\right) & \mu\left(\mu_{3,4}, \mu_{6,7}\right) & \mu\left(\mu_{8,9,10}, \mu_{10,11}\right) & \mu_{3,7} \\ \mu_{1,12} & \mu\left(\mu_{3,4}, c_{3}\right) & \mu\left(\mu_{10,12}, c_{10}\right) & \mu\left(\mu_{6,7}, c_{7}\right)\end{array}\right]$.

Proof. This is straightforward using the explicit form of the matrices $\boldsymbol{E}_{1,2}, \ldots, \boldsymbol{E}_{4,2}$.

Figure 10 illustrates a geometric interpretation of the subdivision algorithm.
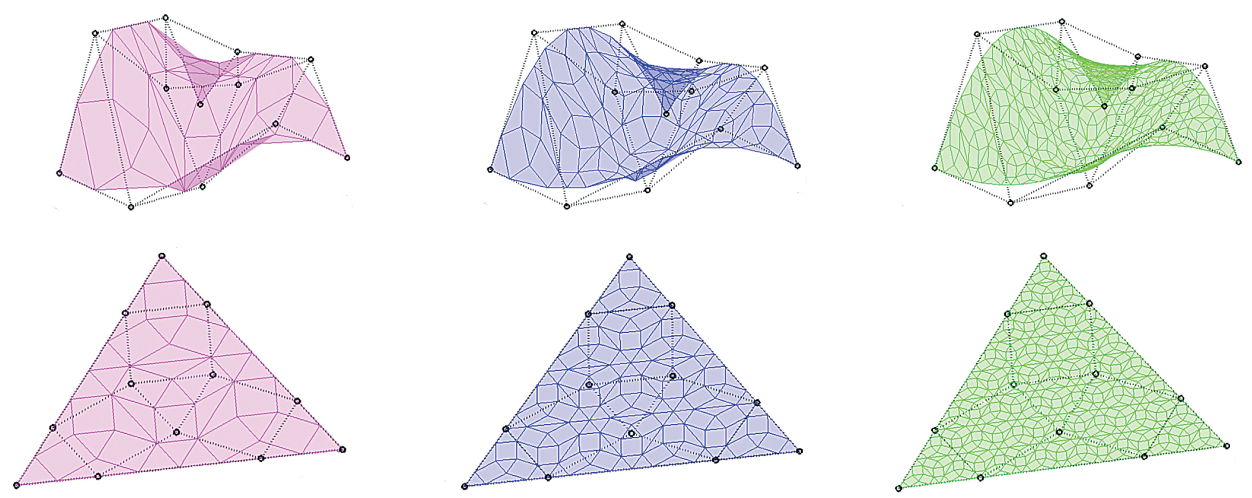

FiguRE 10. Subdivided domain mesh and surfaces. Left: one level of subdivision; center: two levels of subdivision; right: three levels of subdivision. 
6. Stability of the S-BAsis And the Distance to the COntrol Points

6.1. Linear and quadratic quasi-interpolant. We recall from (3.7) that the domain points $\boldsymbol{m}_{j, d}$ are specified as,

$$
\boldsymbol{x}=\sum_{j=1}^{n_{d}} \boldsymbol{m}_{j, d} S_{j, d}(\boldsymbol{x}), \text { for } d=1,2 \text { and } \boldsymbol{x} \in \Delta .
$$

Of the many possible quasi-interpolants to consider, we examine only $Q_{d}$ : $C(\Delta) \rightarrow \mathbb{S}_{d}$ given by

$$
\begin{aligned}
Q_{1} f & :=\sum_{j=1}^{10}\left(\lambda_{j, 1} f\right) S_{j, 1}, \quad \text { where } \lambda_{j, 1} f:=f\left(\boldsymbol{m}_{j, 1}\right), \\
Q_{2} f & :=\sum_{j=1}^{12}\left(\lambda_{j, 2} f\right) S_{j, 2}, \text { where } \lambda_{j, 2} f:=2 f\left(\boldsymbol{m}_{j, 2}\right)-\frac{1}{2} f\left(\boldsymbol{p}_{j, 2,1}^{*}\right)-\frac{1}{2} f\left(\boldsymbol{p}_{j, 2,2}^{*}\right),
\end{aligned}
$$

where, $\boldsymbol{m}_{j, d}, \boldsymbol{p}_{j, 2,1}^{*}$ and $\boldsymbol{p}_{j, 2,2}^{*}$ are given in (3.8) and (3.3). We next show that the $\lambda_{j, d}$ 's are dual functionals with respect to the S-spline basis.

Lemma 6.1. For $d=1,2, i=1, \ldots, 12$, and $j=1, \ldots, n_{d}, \lambda_{i, d} S_{j, d}=\delta_{i j}$.

Proof. The case $d=1$ follows trivially because the corresponding basis functions are hat functions. Now, consider the case $d=2$, for which the value of the $j$ th quadratic S-spline at the $i$ th linear domain point is $\boldsymbol{\Upsilon}_{1}(i, j)$ in the matrix below:

$$
\boldsymbol{\Upsilon}_{1}:=\left[S_{j, 2}\left(\boldsymbol{m}_{i, 1}\right)\right]=\left[\begin{array}{cccccccccccc}
1 & 0 & 0 & 0 & 0 & 0 & 0 & 0 & 0 & 0 & 0 & 0 \\
0 & 0 & 0 & 0 & 1 & 0 & 0 & 0 & 0 & 0 & 0 & 0 \\
0 & 0 & 0 & 0 & 0 & 0 & 0 & 0 & 1 & 0 & 0 & 0 \\
0 & \frac{1}{2} & 0 & \frac{1}{2} & 0 & 0 & 0 & 0 & 0 & 0 & 0 & 0 \\
0 & 0 & 0 & 0 & 0 & \frac{1}{2} & 0 & \frac{1}{2} & 0 & 0 & 0 & 0 \\
0 & 0 & 0 & 0 & 0 & 0 & 0 & 0 & 0 & \frac{1}{2} & 0 & \frac{1}{2} \\
0 & \frac{1}{8} & \frac{3}{8} & 0 & 0 & 0 & 0 & 0 & 0 & 0 & \frac{3}{8} & \frac{1}{8} \\
0 & 0 & \frac{3}{8} & \frac{1}{8} & 0 & \frac{1}{8} & \frac{3}{8} & 0 & 0 & 0 & 0 & 0 \\
0 & 0 & 0 & 0 & 0 & 0 & \frac{3}{8} & \frac{1}{8} & 0 & \frac{1}{8} & \frac{3}{8} & 0 \\
0 & 0 & \frac{1}{3} & 0 & 0 & 0 & \frac{1}{3} & 0 & 0 & 0 & \frac{1}{3} & 0
\end{array}\right] .
$$

Analogously, $\mathbf{\Upsilon}_{2}(i, j)$ below describes the value of the $j$ th quadratic S-spline at the $i$ th quadratic domain point:

$$
\boldsymbol{\Upsilon}_{2}:=\left[S_{j, 2}\left(\boldsymbol{m}_{i, 2}\right)\right]=\left[\begin{array}{cccccccccccc}
1 & 0 & 0 & 0 & 0 & 0 & 0 & 0 & 0 & 0 & 0 & 0 \\
\frac{1}{4} & \frac{5}{8} & 0 & \frac{1}{8} & 0 & 0 & 0 & 0 & 0 & 0 & 0 & 0 \\
0 & \frac{1}{8} & \frac{7}{12} & \frac{1}{8} & 0 & 0 & \frac{1}{12} & 0 & 0 & 0 & \frac{1}{12} & 0 \\
0 & \frac{1}{8} & 0 & \frac{5}{8} & \frac{1}{4} & 0 & 0 & 0 & 0 & 0 & 0 & 0 \\
0 & 0 & 0 & 0 & 1 & 0 & 0 & 0 & 0 & 0 & 0 & 0 \\
0 & 0 & 0 & 0 & \frac{1}{4} & \frac{5}{8} & 0 & \frac{1}{8} & 0 & 0 & 0 & 0 \\
0 & 0 & \frac{1}{12} & 0 & 0 & \frac{1}{8} & \frac{7}{12} & \frac{1}{8} & 0 & 0 & \frac{1}{12} & 0 \\
0 & 0 & 0 & 0 & 0 & \frac{1}{8} & 0 & \frac{5}{8} & \frac{1}{4} & 0 & 0 & 0 \\
0 & 0 & 0 & 0 & 0 & 0 & 0 & 0 & 1 & 0 & 0 & 0 \\
0 & 0 & 0 & 0 & 0 & 0 & 0 & 0 & \frac{1}{4} & \frac{5}{8} & 0 & \frac{1}{8} \\
0 & 0 & \frac{1}{12} & 0 & 0 & 0 & \frac{1}{12} & 0 & 0 & \frac{1}{8} & \frac{7}{12} & \frac{1}{8} \\
\frac{1}{4} & 0 & 0 & 0 & 0 & 0 & 0 & 0 & 0 & \frac{1}{8} & 0 & \frac{5}{8}
\end{array}\right] .
$$


The value of $\left[\lambda_{i, 2} S_{1,2}, \ldots, \lambda_{i, 2} S_{12,2}\right]$ is found by combining row $i$ of $\boldsymbol{\Upsilon}_{2}$ with suitable rows of the matrices $\boldsymbol{\Upsilon}_{1}$ and $\boldsymbol{\Upsilon}_{2}$. For example,

$$
\begin{aligned}
2 \Upsilon_{2}(2,:)-\left(\Upsilon_{1}(1,:)+\Upsilon_{1}(4,:)\right) / 2 & =[0,1,0,0,0,0,0,0,0,0,0,0], \\
2 \Upsilon_{2}(3,:)-\left(\Upsilon_{1}(4,:)+\Upsilon_{1}(10,:)\right) / 2 & =[0,0,1,0,0,0,0,0,0,0,0,0],
\end{aligned}
$$

showing that $\lambda_{2,2} S_{j, 2}=\delta_{2, j}$, and $\lambda_{3,2} S_{j, 2}=\delta_{3, j}$.

6.2. Stability of the quadratic S-spline basis. For each $j$ we let $\Omega_{j, d}:=$ $\operatorname{supp}\left(S_{j, d}\right)$ be the support of $S_{j, d}$. The following theorem shows the S-spline basis achieves stability in the $L_{\infty}$ norm.

Theorem 6.2. For any $f_{d}=s_{d}^{T} c \in \mathbb{S}_{d}$,

$$
K_{d}^{-1}\|\boldsymbol{c}\|_{\infty} \leq\left\|f_{d}\right\|_{L_{\infty}(\Delta)} \leq\|\boldsymbol{c}\|_{\infty}, \quad d=0,1,2,
$$

where $K_{0}=K_{1}=1$ and $K_{2}=3$. Furthermore, the constants $K_{i}$ are the best possible.

Proof. Since the S-splines form a nonnegative partition of unity the upper bound is elementary. Also the values for $K_{0}$ and $K_{1}$ follow easily. Using Lemma 6.1 we see that $c_{j}=2 f\left(\boldsymbol{m}_{j, 2}\right)-\frac{1}{2} f\left(\boldsymbol{p}_{j, 2,1}^{*}\right)-\frac{1}{2} f\left(\boldsymbol{p}_{j, 2,2}^{*}\right)$ for all $j$. Since $\boldsymbol{p}_{j, 2,1}^{*}, \boldsymbol{p}_{j, 2,2}^{*}$ and $\boldsymbol{m}_{j, 2}$ all belong to $\Omega_{j, d}$ we obtain for each $j$,

$$
\left|c_{j}\right| \leq 3\left\|f_{d}\right\|_{L_{\infty}\left(\Omega_{j, d}\right)} \leq 3\left\|f_{d}\right\|_{L_{\infty}(\Delta)},
$$

thus establishing the lower bound. Equality follows by choosing $f$ so that it reduces to the quadratic Chebyshev polynomial, on say, the edge $\left[\boldsymbol{p}_{1}, \boldsymbol{p}_{4}\right]$.

Consider next the $L_{q}$ norm. The next theorem and corollary show that a scaled S-basis is stable in the $L_{q}$ norm.

Theorem 6.3. For any $f_{d}=\boldsymbol{s}_{d}^{T} \boldsymbol{c} \in \mathbb{S}_{d}$ and $q \geq 1$ there is a constant $C_{d}$ depending only on d such that,

$$
C_{d}^{-1}\|\boldsymbol{c}\|_{q, \sigma} \leq\left\|f_{d}\right\|_{L_{q}(\Delta)} \leq\|\boldsymbol{c}\|_{q, \sigma}, \quad d=0,1,2
$$

where

$$
\|\boldsymbol{c}\|_{q, \sigma}:=\left(\sum_{j}\left|c_{j}\right|^{q} \sigma_{j, d}\right)^{1 / q}, \quad \sigma_{j, d}:=\int_{\Delta} S_{j, d}=\frac{v\left(\Omega_{j, d}\right)}{\nu_{d}} .
$$

Proof. We first show that there is a constant $\kappa$ depending only on $d$ such that for any $g \in \mathbb{S}_{d}$,

$$
|g(\boldsymbol{x})| \leq \frac{\kappa}{v\left(\Omega_{j, d}\right)} \int_{\Omega_{j, d}}|g(\boldsymbol{z})| d \boldsymbol{z}, \quad \boldsymbol{x} \in \Omega_{j, d}, \quad j=1, \ldots, n_{d} .
$$

To show this, observe that for all $k, j$,

$$
v\left(\Omega_{j, d}\right) \leq \frac{3}{4} v(\Delta) \leq \frac{3}{4} \cdot 24 v\left(\Delta_{k}\right)=18 v\left(\Delta_{k}\right) .
$$

If $\boldsymbol{x} \in \Omega_{j, d}$, then $\boldsymbol{x} \in \Delta_{k}$ for some $k$. Now $g$ is a polynomial on $\Delta_{k}$ and by equivalence of norms there is a constant $C$ depending only on $d$ such that

$$
|g(\boldsymbol{x})| \leq \frac{C}{v\left(\Delta_{k}\right)} \int_{\Delta_{k}}|g(\boldsymbol{z})| d \boldsymbol{z} \leq \frac{18 C}{v\left(\Omega_{j, d}\right)} \int_{\Omega_{j, d}}|g(\boldsymbol{z})| d \boldsymbol{z},
$$

which proves (6.7). 
The rest of the proof is based on standard $L_{q}$ gymnastics. For the upper inequality, using the relation $1 / q+1 / q^{\prime}=1$, and Hölder's inequality for sums

$$
\|f\|_{L_{q}(\Delta)}^{q}=\int_{\Delta}\left(\sum_{j} c_{j} S_{j}^{1 / q} S_{j}^{1 / q^{\prime}}\right)^{q} \leq \sum_{j}\left|c_{j}\right|^{q} \int_{\Delta} S_{j, d}=\|\boldsymbol{c}\|_{q, \sigma}^{q} .
$$

For the lower inequality, using (6.4), (6.7) and Hölder's inequality for integrals over $\Omega_{j, d}$

$$
\sigma_{j, d}^{1 / q}\left|c_{j}\right| \leq K_{d} \sigma_{j, d}^{1 / q}\|f\|_{L_{\infty}\left(\Omega_{j, d}\right)} \leq \frac{\kappa K_{d}}{\sigma_{j, d}^{1-1 / q}}\|f\|_{L_{1}\left(\Omega_{j, d}\right)} \leq \kappa_{1}\|f\|_{L_{q}\left(\Omega_{j, d}\right)} .
$$

Here $\kappa_{1}$ is a constant depending only on $d$. Raising this to the $q$ th power and summing over $j$ gives

$$
\|\boldsymbol{c}\|_{q, \sigma}^{q} \leq \kappa_{1}^{q} \sum_{j}\|f\|_{L_{q}\left(\Omega_{j, d}\right)}^{q} \leq \kappa_{2}^{q}\|f\|_{L_{q}(\Delta)}^{q}
$$

for some constant $\kappa_{2}$ depending only on $d$. Taking the $q$ th root proves the lower inequality with $C_{d}=\kappa_{2}$.

By scaling the S-basis appropriately we can remove the weights in the discrete $l_{q}$ norms.

Corollary 6.4. Define $L_{q}$ scaled $S$-splines by

$$
S_{j, d}^{[q]}:=\sigma_{j, d}^{-1 / q} S_{j, d}, \quad j=1, \ldots, n_{d}, \quad 1 \leq q \leq \infty,
$$

where $\sigma_{j, q}=\int_{\Delta} S_{j, d}$, and set $\boldsymbol{s}_{d}^{[q] T}=\left[S_{1, d}^{[q]}, \ldots, S_{n_{d}, d}^{[q]}\right]$. For any $f_{d}=\boldsymbol{s}_{d}^{[q] T} \boldsymbol{c} \in \mathbb{S}_{d}$ and $q \geq 1$ there is a constant $C_{d}$ depending only on $d$ such that

$$
C_{d}^{-1}\|\boldsymbol{c}\|_{q} \leq\left\|f_{d}\right\|_{L_{q}(\Delta)} \leq\|\boldsymbol{c}\|_{q}, \quad d=0,1,2 .
$$

Notice that the constant $C_{d}$ does not depend on the shape and size of the triangle $\Delta$.

6.3. Distance between a surface and its control points. We show that the distance between a surface and its governing control points is $O\left(h^{2}\right)$, where $h$ is the length of the longest edge of the triangle $\Delta$.

Theorem 6.5. For any $f_{2}=s_{2}^{T} \boldsymbol{c} \in \mathbb{S}_{2}$ there is a constant $K$ independent of $h$ such that

$$
\left|c_{j}-f_{2}\left(\boldsymbol{m}_{j, 2}\right)\right| \leq K h^{2}, j=1, \ldots, 12 .
$$

Proof. Recall from the proof of Theorem 6.2 that $c_{j}=2 f_{2}\left(\boldsymbol{m}_{j, 2}\right)-\frac{1}{2} f_{2}\left(\boldsymbol{p}_{j, 2,1}^{*}\right)-$ $\frac{1}{2} f_{2}\left(\boldsymbol{p}_{j, 2,2}^{*}\right)$ for all $j$. Define

$$
g_{j}(t):=f_{2}\left(\boldsymbol{p}_{j, 2,1}^{*}+t \frac{\boldsymbol{p}_{j, 2,2}^{*}-\boldsymbol{p}_{j, 2,1}^{*}}{h_{j}}\right), \quad h_{j}:=\left\|\boldsymbol{p}_{j, 2,2}^{*}-\boldsymbol{p}_{j, 2,1}^{*}\right\|_{2}, \quad t \in\left[0, h_{j}\right] .
$$

Observe that $g_{j}$ is a quadratic polynomial since $\boldsymbol{p}_{j, 2,1}^{*}, \boldsymbol{p}_{j, 2,2}^{*}, \boldsymbol{m}_{j, 2}$ lie on an edge of a subtriangle, so,

$$
\begin{aligned}
2\left|c_{j}-f_{2}\left(\boldsymbol{m}_{j, 2}\right)\right| & =\left|2 f_{2}\left(\boldsymbol{m}_{j, 2}\right)-f_{2}\left(\boldsymbol{p}_{j, 2,1}^{*}\right)-f_{2}\left(\boldsymbol{p}_{j, 2,2}^{*}\right)\right| \\
& =\left|2 g_{j}\left(\frac{h_{j}}{2}\right)-g_{j}(0)-g_{j}\left(h_{j}\right)\right|=\frac{h_{j}^{2}}{4}\left|g_{j}^{\prime \prime}\left(\frac{h_{j}}{2}\right)\right| .
\end{aligned}
$$




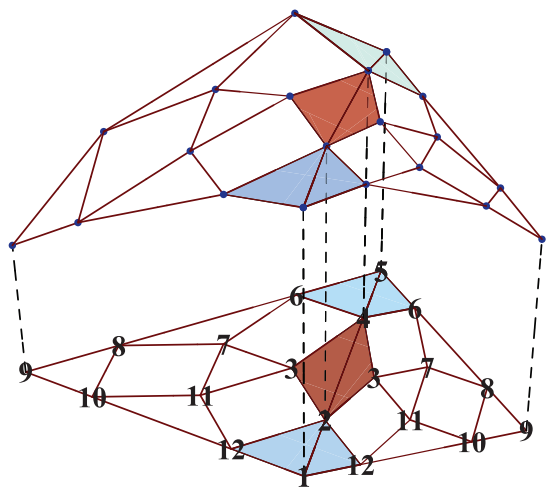

Figure 11. Constraints on the three control mesh regions of each surface for $C^{1}$ continuity.

Since $\left\|\frac{\boldsymbol{p}_{j, 2,2}^{*}-\boldsymbol{p}_{j, 2,1}^{*}}{h_{j}}\right\|_{2}=1$, we can bound $\left|g_{j}^{\prime \prime}\left(\frac{h_{j}}{2}\right)\right|$ independent of $h_{j}$, and then claim the desired result.

\section{Smooth surfaces Joins: Conditions on PS12 Refinements of TRIANGULATIONS}

Let $\mathcal{T}$ be a triangulation of a polygonal region $\Omega$ in the plane. Further let $\mathcal{T}_{P S 12}$ be the refined triangulation obtained by splitting each $T \in \mathcal{T}$ using the PowellSabin 12 split. For $d=0,1,2$ and $-1 \leq r \leq s<d$ let $\mathbb{S}_{d}^{r, s}\left(\mathcal{T}_{P S 12}\right)$ be the space of functions $f$ in $C^{r}(\Omega)$, such that the restriction to any triangle of $\mathcal{T}_{P S 12}$ is a degree $d$ polynomial and $f \in C^{s}(T)$ for each $T \in \mathcal{T}$. We set $\mathbb{S}_{d}^{r}\left(\mathcal{T}_{P S 12}\right):=\mathbb{S}_{d}^{r, d-1}\left(\mathcal{T}_{P S 12}\right)$. Over each $T_{i} \in \mathcal{T}$ we have an S-spline basis $\left\{S_{j, d, i}\right\}$ of degree $d$. In particular, $S_{j, d, i} \in C^{d-1}\left(T_{i}\right)$. For $r=-1$ the collection $\left\{\left\{S_{j, d, i}\right\}_{j}\right\}_{i}$ constitutes a basis for $\mathbb{S}_{d}^{-1}\left(\mathcal{T}_{P S 12}\right)$

We seek conditions on the S-spline coefficients to guarantee $C^{r}$ continuity $r=$ 0,1 , across a common edge (cf. Figure 11).

Theorem 7.1. Suppose $\mathcal{T}=\left\{T_{1}, T_{2}\right\}$ share a common edge $\operatorname{ch}\left(\left\{\boldsymbol{p}_{1}, \boldsymbol{p}_{2}\right\}\right)$. We use the quadratic domain points $\boldsymbol{m}_{j, 2}$ to number the vertices, so that

$T_{1}=\operatorname{ch}\left(\left\{\boldsymbol{m}_{1,2}^{1}, \boldsymbol{m}_{5,2}^{1}, \boldsymbol{m}_{9,2}^{1}\right\}\right)$ and $T_{2}=\operatorname{ch}\left(\left\{\boldsymbol{m}_{1,2}^{1}, \boldsymbol{m}_{5,2}^{1}, \boldsymbol{m}_{9,2}^{2}\right\}\right)$, (cf. Figure 11). Let $f \in \mathbb{S}_{d}^{-1}\left(\mathcal{T}_{P S 12}\right)$ be a spline of degree $d \in\{1,2\}$ and let

$$
f_{1}:=\left.f\right|_{T_{1}}=\sum_{j} c_{j} S_{j, d, 1}, \quad f_{2}:=\left.f\right|_{T_{2}}=\sum_{j} d_{j} S_{j, d, 2}
$$

be the restrictions of $f$ to $T_{i}$ for $i=1,2$.

(1) $f \in \mathbb{S}_{1}^{0}\left(\mathcal{T}_{P S 12}\right)$ if and only if $c_{j}=d_{j}$ for $j=1,2,4$.

(2) $f \in \mathbb{S}_{2}^{0}\left(\mathcal{T}_{P S 12}\right)$ if and only if $c_{j}=d_{j}$ for $j=1,2,4,5$.

(3) $f \in \mathbb{S}_{2}^{1}\left(\mathcal{T}_{P S 12}\right)$ if and only if $f \in \mathbb{S}_{2}^{0}\left(\mathcal{T}_{P S 12}\right)$ and in addition

$$
\begin{aligned}
d_{12} & =\beta_{1} c_{1}+\beta_{2} c_{2}+\beta_{3} c_{12}, \\
d_{3} & =\frac{2 \beta_{1}+\beta_{2}}{3} c_{2}+\frac{\beta_{1}+2 \beta_{2}}{3} c_{4}+\beta_{3} c_{3}, \\
d_{6} & =\beta_{1} c_{4}+\beta_{2} c_{5}+\beta_{3} c_{6},
\end{aligned}
$$


where $\boldsymbol{m}_{9,2}^{2}=\beta_{1} \boldsymbol{m}_{1,2}^{1}+\beta_{2} \boldsymbol{m}_{5,2}^{1}+\beta_{3} \boldsymbol{m}_{9,2}^{1}$, so that $\beta_{1}, \beta_{2}, \beta_{3}$ are the barycentric coordinates of the vertex $\boldsymbol{m}_{9,2}^{2}$ of $T_{2}$ with respect to $T_{1}$.

Proof. We show only the quadratic case and consider first $C^{0}$ continuity.

By the edge property (2.8) the restriction of $f_{i}$ to the edge $\operatorname{ch}\left(\left\{\boldsymbol{m}_{1,2}^{1}, \boldsymbol{m}_{5,2}^{1}\right\}\right)$ is a sum of univariate quadratic B-splines $\left\{B_{j, 2}\right\}_{j=1}^{4}$ over the common knot vector $[0,0,0,1 / 2,1,1,1]$

$$
f_{1}=c_{1} B_{1,2}+c_{2} B_{2,2}+c_{4} B_{3,2}+c_{5} B_{4,1}, \quad f_{2}=d_{1} B_{1,2}+d_{2} B_{2,2}+d_{4} B_{3,2}+d_{5} B_{4,1} .
$$

Therefore, the surface is continuous if and only if these curves are the same, a condition that follows if and only if $c_{j}=d_{j}$ for $j=1,2,4,5$.

We proceed to discuss conditions on $f_{i}$ that ensure $C^{1}$ continuity for a quadratic $f$ given we already have $C^{0}$ continuity, that is, $c_{j}=d_{j}$ for $j=1,2,4,5$. $C^{1}$ continuity holds if and only if

$\tau_{1}(t):=D_{\boldsymbol{u}} f_{1}\left((1-t) \boldsymbol{m}_{1,2}^{1}+t \boldsymbol{m}_{5,2}^{1}\right)=D_{\boldsymbol{u}} f_{2}\left((1-t) \boldsymbol{m}_{1,2}^{1}+t \boldsymbol{m}_{5,2}^{1}\right)=: \tau_{2}(t), \quad 0 \leq t \leq 1$,

where $\boldsymbol{u}:=\boldsymbol{m}_{9,2}^{2}-\boldsymbol{m}_{1,2}^{1}$ is the cross-boundary direction chosen to be along the edge $\operatorname{ch}\left(\left\{\boldsymbol{m}_{1,2}^{1}, \boldsymbol{m}_{9,2}^{2}\right\}\right)$ of $T_{2}$. To apply the differentiation formula in Theorem 3.9 we observe that for this $\boldsymbol{u}$ the $\alpha$ 's in the theorem are given by $\boldsymbol{\alpha}^{1}=\left[\beta_{1}-1, \beta_{2}, \beta_{3}\right]^{T}$ for $T_{1}$ and $\boldsymbol{\alpha}^{2}=[-1,0,1]^{T}$ for $T_{2}$. By the differentiation formula (3.25), Table 2 , and the edge restriction formula (2.7), we get

$$
\begin{aligned}
\frac{1}{2} \tau_{1} & =\left(2 \alpha_{2}^{1} c_{2,1}+2 \alpha_{3}^{1} c_{12,1}\right) B_{1,1}+\left(\alpha_{1,3}^{1} c_{2,3}+\alpha_{2,3}^{1} c_{4,3}\right) B_{2,1} \\
& +\left(2 \alpha_{1}^{1} c_{4,5}+2 \alpha_{3}^{1} c_{6,5}\right) B_{3,1}
\end{aligned}
$$

and

$$
\begin{aligned}
\frac{1}{2} \tau_{2} & =\left(2 \alpha_{2}^{2} d_{2,1}+2 \alpha_{3}^{2} d_{12,1}\right) B_{1,1}+\left(\alpha_{1,3}^{2} d_{2,3}+\alpha_{2,3}^{2} d_{4,3}\right) B_{2,1} \\
& +\left(2 \alpha_{1}^{2} d_{4,5}+2 \alpha_{3}^{2} d_{6,5}\right) B_{3,1}
\end{aligned}
$$

where $B_{j, 1}, j=1,2,3$ are the partition of unity linear B-splines on the knot vector $[0,0,1 / 2,1,1]$. By linear independence of these B-splines and since $\tau_{1}=\tau_{2}$,

$$
\begin{aligned}
2 \alpha_{2}^{1} c_{2,1}+2 \alpha_{3}^{1} c_{12,1} & =2 \alpha_{2}^{2} d_{2,1}+2 \alpha_{3}^{2} d_{12,1}, \\
\alpha_{1,3}^{1} c_{2,3}+\alpha_{2,3}^{1} c_{4,3} & =\alpha_{1,3}^{2} d_{2,3}+\alpha_{2,3}^{2} d_{4,3}, \\
2 \alpha_{1}^{1} c_{4,5}+2 \alpha_{3}^{1} c_{6,5} & =2 \alpha_{1}^{2} d_{4,5}+2 \alpha_{3}^{2} d_{6,5} .
\end{aligned}
$$

Substituting in values for $\boldsymbol{\alpha}^{1}, \boldsymbol{\alpha}^{2}, c_{i, j}$, and $d_{i, j}$ yields

$$
\begin{aligned}
\beta_{2}\left(c_{2}-c_{1}\right)+\beta_{3}\left(c_{12}-c_{1}\right) & =d_{12}-d_{1}=d_{12}-c_{1}, \\
\left(\beta_{1}-1-\beta_{3}\right)\left(c_{2}-c_{3}\right)+\left(\beta_{2}-\beta_{3}\right)\left(c_{4}-c_{3}\right) & =-2\left(d_{2}-d_{3}\right)-\left(d_{4}-d_{3}\right) \\
& =3 d_{3}-2 c_{2}-c_{4} \\
\left(\beta_{1}-1\right)\left(c_{4}-c_{5}\right)+\beta_{3}\left(c_{6}-c_{5}\right) & =-\left(d_{4}-d_{5}\right)+\left(d_{6}-d_{5}\right)=d_{6}-c_{4} .
\end{aligned}
$$

Solving for $d_{12}, d_{3}, d_{6}$ and simplifying we obtain (7.2). 
The equations at (7.2) give $d_{j}$ as affine combinations of three neighboring points in $T_{1}$ for $j=3,6,12$. Hence, the $C^{1}$ conditions (7.2), illustrated in Figure 11, also show that each of the sets of four coefficients in (17.2) can be used to define coplanar points located at the quadratic domain points. More precisely,

Corollary 7.2. $C^{1}$ constraints for the control points can be written as

$$
\begin{aligned}
\left(\boldsymbol{m}_{12,2}^{2}, d_{12}\right) & =\beta_{1}\left(\boldsymbol{m}_{1,2}^{1}, c_{1}\right)+\beta_{2}\left(\boldsymbol{m}_{2,2}^{1}, c_{2}\right)+\beta_{3}\left(\boldsymbol{m}_{12,2}^{1}, c_{12}\right), \\
\left(\boldsymbol{m}_{3,2}^{2}, d_{3}\right) & =\frac{2 \beta_{1}+\beta_{2}}{3}\left(\boldsymbol{m}_{2,2}^{1}, c_{2}\right)+\frac{\beta_{1}+2 \beta_{2}}{3}\left(\boldsymbol{m}_{4,2}^{1}, c_{4}\right)+\beta_{3}\left(\boldsymbol{m}_{3,2}^{1}, c_{3}\right), \\
\left(\boldsymbol{m}_{6,2}^{2}, d_{6}\right) & =\beta_{1}\left(\boldsymbol{m}_{4,2}^{1}, c_{4}\right)+\beta_{2}\left(\boldsymbol{m}_{5,2}^{1}, c_{5}\right)+\beta_{3}\left(\boldsymbol{m}_{6,2}^{1}, c_{6}\right) .
\end{aligned}
$$

Proof. Since the triangles $\operatorname{ch}\left(\left\{\boldsymbol{m}_{1,2}^{1}, \boldsymbol{m}_{2,2}^{1}, \boldsymbol{m}_{12,2}^{1}\right\}\right)$ and $\operatorname{ch}\left(\left\{\boldsymbol{m}_{1,2}^{1}, \boldsymbol{m}_{5,2}^{1}, \boldsymbol{m}_{9,2}^{1}\right\}\right)$ are similar and $\boldsymbol{m}_{9,2}^{2}=\beta_{1} \boldsymbol{m}_{1,1}^{1}+\beta_{2} \boldsymbol{m}_{5,1}^{1}+\beta_{3} \boldsymbol{m}_{9,1}^{1}$ it follows easily that $\boldsymbol{m}_{12,2}^{2}=$ $\beta_{1} \boldsymbol{m}_{1,2}^{1}+\beta_{2} \boldsymbol{m}_{2,2}^{1}+\beta_{3} \boldsymbol{m}_{12,2}^{1}$. Similarly, $\boldsymbol{m}_{6,2}^{2}=\beta_{1} \boldsymbol{m}_{4,2}^{1}+\beta_{2} \boldsymbol{m}_{5,2}^{1}+\beta_{3} \boldsymbol{m}_{6,2}^{1}$. We leave the proof that $\boldsymbol{m}_{3,2}^{2}=\frac{2 \beta_{1}+\beta_{2}}{3} \boldsymbol{m}_{2,2}^{1}+\frac{\beta_{1}+2 \beta_{2}}{3} \boldsymbol{m}_{4,2}^{1}+\beta_{3} \boldsymbol{m}_{3,2}^{1}$ to the reader.

\section{Approximation methods}

Using the S-spline basis we can consider the usual linear approximation methods like various types of interpolation, least squares, and quasi-interpolants. In this section we discuss two types of interpolation and return to the quadratic quasiinterpolant defined in (6.1).

8.1. Lagrange interpolation. We consider first Lagrange interpolation. Given 12 distinct points $\boldsymbol{x}_{1}, \ldots, \boldsymbol{x}_{12}$ in the triangle $\Delta$ and 12 real numbers $z_{1}, \ldots, z_{12}$. The problem is to find $g \in \mathbb{S}_{2}$ such that,

$$
g\left(\boldsymbol{x}_{i}\right)=z_{i}, \text { for } i=1, \ldots, 12 .
$$

In general the collocation matrix $\boldsymbol{C}:=\left(S_{j, 2}\left(\boldsymbol{x}_{i}\right)\right)_{i, j=1}^{12}$ can be singular. This happens, for example, if there are more than 6 points in one subtriangle.

We show that interpolation at the quadratic domain points is uniquely solvable.

Theorem 8.1. The collocation matrix $\boldsymbol{C}_{2}:=\left(S_{j, 2}\left(\boldsymbol{m}_{i, 2}\right)\right)_{i, j=1}^{12}$ for interpolation at domain points is nonsingular. Moreover, $\left\|\boldsymbol{C}_{2}^{-1}\right\|_{\infty}=28 / 9$. Hence, if $g=$ $\sum_{j=1}^{12} c_{j} S_{j, 2}$ is the interpolant to $\boldsymbol{z} \in \mathbb{R}^{12}$ then $\|g\|_{\infty} \leq\|\boldsymbol{c}\|_{\infty} \leq \frac{28}{9}\|\boldsymbol{z}\|_{\infty}$, where $\boldsymbol{c}:=\left[c_{1}, \ldots, c_{12}\right]^{T}$. Thus the interpolation at domain points is stable independently of the geometry of the triangle.

Proof. The matrix $\boldsymbol{C}_{2}$ is given by (6.2) . Because it is strictly diagonally dominant, it must be nonsingular. The elements of $\boldsymbol{C}_{2}$ are independent of the location and shape of $\Delta$ so the inverse of $\boldsymbol{C}_{2}$ can be easily computed symbolically. From this calculation it follows that $\left\|\boldsymbol{C}_{2}^{-1}\right\|_{\infty}=28 / 9$. The S-splines form a nonnegative partition of unity and the inequality $\|g\|_{\infty} \leq\|\boldsymbol{c}\|_{\infty}$ follows. Since $\boldsymbol{C}_{2} \boldsymbol{c}=\boldsymbol{z}$ we find $\boldsymbol{c}=\boldsymbol{C}_{2}^{-1} \boldsymbol{z}$ and hence $\|\boldsymbol{c}\|_{\infty} \leq \frac{28}{9}\|\boldsymbol{z}\|_{\infty}$. 
8.2. Hermite interpolation. The classical interpolation problem in finite elements over the PS12-split is to interpolate values and gradients at the vertices and unit normals at the midpoint of the edges. These interpolation conditions can be described as linear functionals $\left\{\rho_{j}\right\}_{j=1}^{12}$, such that,

$$
\begin{aligned}
\boldsymbol{\rho}(f) & :=\left[\rho_{1}(f), \ldots, \rho_{12}(f)\right]^{T} \\
& =\left[f\left(\boldsymbol{p}_{1}\right), f_{x}\left(\boldsymbol{p}_{1}\right), f_{y}\left(\boldsymbol{p}_{1}\right), f_{\boldsymbol{n}_{3}}\left(\boldsymbol{p}_{4}\right), f\left(\boldsymbol{p}_{2}\right), f_{x}\left(\boldsymbol{p}_{2}\right), \ldots, f_{y}\left(\boldsymbol{p}_{3}\right), f_{\boldsymbol{n}_{2}}\left(\boldsymbol{p}_{6}\right)\right]^{T},
\end{aligned}
$$

where $f_{\boldsymbol{n}_{i}}$ is the derivative in the unit normal direction to the edge opposite $\boldsymbol{p}_{i}=$ $\left[x_{1}, y_{i}\right]^{T}$ pointing inwards, $i=1,2,3$. The coefficients in the interpolant $g=\boldsymbol{s}^{T} \boldsymbol{c}$ are solutions of the linear system

$$
A c=\rho(f),
$$

where the rows of $\boldsymbol{A} \in \mathbb{R}^{12,12}$ are given by

(8.1)

$$
\begin{aligned}
& \boldsymbol{A}(1,:)=e_{1} \text {, } \\
& \boldsymbol{A}(2,:)=4\left[\begin{array}{llllllllllll}
y_{2}-y_{3} & y_{3}-y_{1} & 0 & 0 & 0 & 0 & 0 & 0 & 0 & 0 & 0 & y_{1}-y_{2}
\end{array}\right] / \delta, \\
& \boldsymbol{A}(3,:)=4\left[\begin{array}{llllllllllll}
x_{3}-x_{2} & x_{1}-x_{3} & 0 & 0 & 0 & 0 & 0 & 0 & 0 & 0 & 0 & x_{2}-x_{1}
\end{array}\right] / \delta, \\
& \boldsymbol{A}(4,:)=\left\|\boldsymbol{p}_{1}-\boldsymbol{p}_{2}\right\|_{2}\left[\begin{array}{lllllllllllll}
0 & 4 \ell_{126} & 6 & 4 \ell_{215} & 0 & 0 & 0 & 0 & 0 & 0 & 0 & 0
\end{array}\right] / \delta \text {, } \\
& \boldsymbol{A}(5,:)=\boldsymbol{e}_{5} \text {, } \\
& \boldsymbol{A}(6,:)=4\left[\begin{array}{llllllllllll}
0 & 0 & 0 & y_{2}-y_{3} & y_{3}-y_{1} & y_{1}-y_{2} & 0 & 0 & 0 & 0 & 0 & 0
\end{array}\right] / \delta, \\
& \boldsymbol{A}(7,:)=4\left[\begin{array}{llllllllllll}
0 & 0 & 0 & x_{3}-x_{2} & x_{1}-x_{3} & x_{2}-x_{1} & 0 & 0 & 0 & 0 & 0 & 0
\end{array}\right] / \delta, \\
& \boldsymbol{A}(8,:)=\left\|\boldsymbol{p}_{2}-\boldsymbol{p}_{3}\right\|_{2}\left[\begin{array}{lllllllllllll}
0 & 0 & 0 & 0 & 0 & 4 \ell_{234} & 6 & 4 \ell_{326} & 0 & 0 & 0 & 0
\end{array}\right] / \delta \text {, } \\
& \boldsymbol{A}(9,:)=\boldsymbol{e}_{9} \text {, } \\
& \boldsymbol{A}(10,:)=4\left[\begin{array}{llllllllllll}
0 & 0 & 0 & 0 & 0 & 0 & 0 & y_{3}-y_{1} & y_{1}-y_{2} & y_{2}-y_{3} & 0 & 0
\end{array}\right] / \delta, \\
& \boldsymbol{A}(11,:)=4\left[\begin{array}{llllllllllll}
0 & 0 & 0 & 0 & 0 & 0 & 0 & x_{1}-x_{3} & x_{2}-x_{1} & x_{3}-x_{2} & 0 & 0
\end{array}\right] / \delta, \\
& \boldsymbol{A}(12,:)=\left\|\boldsymbol{p}_{3}-\boldsymbol{p}_{1}\right\|_{2}\left[\begin{array}{llllllllllll}
0 & 0 & 0 & 0 & 0 & 0 & 0 & 0 & 0 & 4 \ell_{315} & 6 & 4 \ell_{134}
\end{array}\right] / \delta \text {, }
\end{aligned}
$$

where

$$
\ell_{i j k}:=\frac{\left(\boldsymbol{p}_{i}-\boldsymbol{p}_{j}\right)^{T}\left(\boldsymbol{p}_{j}-\boldsymbol{p}_{k}\right)}{\left(\boldsymbol{p}_{i}-\boldsymbol{p}_{j}\right)^{T}\left(\boldsymbol{p}_{i}-\boldsymbol{p}_{j}\right)}, \quad \delta:=\left|\begin{array}{ccc}
1 & 1 & 1 \\
x_{1} & x_{2} & x_{3} \\
y_{1} & y_{2} & y_{3}
\end{array}\right|
$$

We note that $\left\|\boldsymbol{p}_{i}-\boldsymbol{p}_{j}\right\|_{2} \ell_{i j k}$ is the length of the projection of $\boldsymbol{p}_{j}-\boldsymbol{p}_{k}$ in the direction of $\boldsymbol{p}_{i}-\boldsymbol{p}_{j}$ and that $\delta$ is twice the signed area of $\Delta$.

The matrix $\boldsymbol{A}$ is sparse, and simple to compute.

Let $\boldsymbol{h}_{2}^{T}=\left[H_{1,2}, \ldots, H_{12,2}\right]^{T}$ be the nodal basis for $\mathbb{S}_{2}$ defined by $\rho_{j}\left(H_{i, 2}\right)=\delta_{i, j}$. Then

$$
\boldsymbol{h}_{2}^{T}=\boldsymbol{s}_{2}^{T} \boldsymbol{A}^{-1}
$$

gives the representation for the nodal basis in terms of the quadratic S-spline basis. We find that columns 1 through 6 and columns 7 through 12 of $\boldsymbol{A}^{-1}$ are given by the two matrices 


$$
\begin{aligned}
& \mathbf{A}^{-1}(:, 1: 6)= \\
& {\left[\begin{array}{cccccc}
1 & 0 & 0 & 0 & 0 & 0 \\
1 & \frac{1}{4} x_{21} & \frac{1}{4} y_{21} & 0 & 0 & 0 \\
-\frac{2}{3} \ell_{126} & \frac{1}{6} x_{12} \ell_{126} & \frac{1}{6} y_{12} \ell_{126} & \delta /\left(6\left\|\boldsymbol{p}_{12}\right\|_{2}\right) & -\frac{2}{3} \ell_{215} & \frac{1}{6} x_{21} \ell_{215} \\
0 & 0 & 0 & 0 & 1 & \frac{1}{4} x_{12} \\
0 & 0 & 0 & 0 & 1 & 0 \\
0 & 0 & 0 & 0 & 1 & \frac{1}{4} x_{32} \\
0 & 0 & 0 & 0 & -\frac{2}{3} \ell_{234} & \frac{1}{6} x_{23} \ell_{234} \\
0 & 0 & 0 & 0 & 0 & 0 \\
0 & 0 & 0 & 0 & 0 & 0 \\
0 & 0 & 0 & 0 & 0 & 0 \\
-\frac{2}{3} \ell_{134} & \frac{1}{6} x_{13} \ell_{134} & \frac{1}{6} y_{13} \ell_{134} & 0 & 0 & 0 \\
1 & \frac{1}{4} x_{31} & \frac{1}{4} y_{31} & 0 & 0 & 0
\end{array}\right],}
\end{aligned}
$$

$$
\begin{aligned}
& \mathbf{A}^{-1}(:, 7: 12)= \\
& \left.\qquad \begin{array}{cccccc}
0 & 0 & 0 & 0 & 0 & 0 \\
0 & 0 & 0 & 0 & 0 & 0 \\
\frac{1}{6} y_{21} \ell_{215} & 0 & 0 & 0 & 0 & 0 \\
\frac{1}{4} y_{12} & 0 & 0 & 0 & 0 & 0 \\
0 & 0 & 0 & 0 & 0 & 0 \\
\frac{1}{4} y_{32} & 0 & 0 & 0 & 0 & 0 \\
\frac{1}{6} y_{23} \ell_{234} & \delta /\left(6\left\|\boldsymbol{p}_{23}\right\|_{2}\right) & -\frac{2}{3} \ell_{326} & \frac{1}{6} x_{32} \ell_{326} & \frac{1}{6} y_{32} \ell_{326} & 0 \\
0 & 0 & 1 & \frac{1}{4} x_{23} & \frac{1}{4} y_{23} & 0 \\
0 & 0 & 1 & 0 & 0 & 0 \\
0 & 0 & 1 & \frac{1}{4} x_{13} & \frac{1}{4} y_{13} & 0 \\
0 & 0 & -\frac{2}{3} \ell_{315} & \frac{1}{6} x_{31} \ell_{315} & \frac{1}{6} y_{31} \ell_{315} & \delta /\left(6\left\|\boldsymbol{p}_{31}\right\|_{2}\right) \\
0 & 0 & 0 & 0 & 0 & 0
\end{array}\right],
\end{aligned}
$$

where $l_{i j k}$ and $\delta$ are given by (8.2), $x_{i j}=x_{i}-x_{j}, y_{i j}=y_{i}-y_{j}$, and $\boldsymbol{p}_{i j}=\boldsymbol{p}_{i}-\boldsymbol{p}_{j}$.

The inverse $\boldsymbol{A}^{-1}$ is also sparse and easy to compute. Further we observe that each position nodal function uses 5 S-spline basis elements, while those for the partial derivative conditions use $4 \mathrm{~S}$-spline basis elements, and the normal derivative ones are the scaled S-spline basis functions with trapezoidal support.

8.3. Quasi-interpolants. Consider the quasi-interpolant $Q_{2}$ given in (6.1) as

$$
Q_{2} f:=\sum_{j=1}^{12}\left(\lambda_{j, 2} f\right) S_{j, 2}, \text { where } \lambda_{j, 2} f:=2 f\left(\boldsymbol{m}_{j, 2}\right)-\frac{1}{2} f\left(\boldsymbol{p}_{j, 2,1}^{*}\right)-\frac{1}{2} f\left(\boldsymbol{p}_{j, 2,2}^{*}\right) .
$$

This is a projection $Q_{2}: C(\Delta) \rightarrow \mathbb{S}_{2}$ since the $\left\{\lambda_{j, 2}\right\}_{j=1}^{12}$ is a dual basis for the S-spline basis. Moreover, it is easy to see that the $L_{\infty}$ norm of $Q_{2}$ is bounded,

$$
\left\|Q_{2}\right\|_{L_{\infty}(\Delta)} \leq 3 \text {. }
$$

Using a standard argument,

$$
\left\|f-Q_{2} f\right\|_{L_{\infty}(\Delta)} \leq K_{r} h^{r} \max _{\nu+\mu=r}\left\|D^{\nu, \mu} f\right\|_{L_{\infty}(\Delta)}, \quad r=1,2,3, \quad f \in C^{r}(\Delta),
$$

where $h$ is the length of the longest side of $\Delta, K_{r}$ depends only on $r$, and $D^{\nu, \mu} f$ means taking $\nu$, respectively $\mu$ derivatives with respect to the first (second) variable. 


\section{CONCLUding REMARKS}

In this paper we introduced the S-spline basis, a B-spline like basis, for a single PS12 macroelement. Locally this basis is a B-spline basis within a single macroelement, but globally it behaves like a Bernstein-Bézier basis across macro-elements. We prove that the S-spline basis has many desirable B-spline properties including that it forms a partition of unity, offers a recurrence to hat functions, provides a Marsden-like identity, enjoys $L_{q}$ and $L_{\infty}$ stability, and has an associated control mesh. The control mesh is unusual in that it is a hybrid with both triangular and quadrilateral connectivity. The distance from a surface to any one of its control points is $O\left(h^{2}\right)$, where $h$ is the length of the longest side of macrotriangle $\Delta$. Furthermore each basis element reduces to a single univariate B-spline on the boundary edges of the macroelement. Using the S-spline basis over each triangle, the PS12split becomes amenable for use in both surface fitting and finite element analysis over an arbitrary triangulation. We have provided a simple, sparse, explicit basis transformation matrix for expressing the nodal basis in terms of the S-spline basis, as well as its sparse inverse. In addition to deriving subdivision algorithms, we also develop pyramidal algorithms both for evaluation and differentiation. They are simple to use for both explicit and parametric macroelements. Explicit conditions for smoothness across a common edge of two macrotriangles are derived in terms of the respective control meshes. These results highlight both the similarities to and differences from the triangular Bernstein-Bézier approach. The ordering of vertices, triangles and domain points used herein changed several times during the development of this work. We recognize that there are many alternative ways for ordering these quantities. For example, the vertices could be numbered by moving around the edges counterclockwise in such a way that $\Delta=\operatorname{ch}\left(\left\{\boldsymbol{p}_{1}, \boldsymbol{p}_{3}, \boldsymbol{p}_{5}\right\}\right)$. In the polynomial Bernstein/Bézier literature, the basis functions are indexed using a scaling of the barycentric coordinates of the triangular domain points [13. Such a scheme could also be adopted for the S-basis, not to mention the myriad other possibilities that might come to mind. Feeling that a naturally convenient scheme will likely arise from further considerations and experiences over a longer time, we opted for a somewhat arbitrary approach that sufficed for our initial purposes.

\section{Appendix A. Proof of Recurrence Relation Theorem 2.3}

Proof. For any knot set $\boldsymbol{X} \in \mathbb{R}^{2, n}$ we use the simplex spline normalizations

$$
\begin{aligned}
& S(\cdot \mid \boldsymbol{X}):=v(\operatorname{ch}(\boldsymbol{X})) Q(\cdot \mid \boldsymbol{X}), \\
& Q(\cdot \mid \boldsymbol{X}):= \begin{cases}\nu_{d}^{-1} M(\cdot \mid \boldsymbol{X}), & \text { if } \operatorname{span}(\boldsymbol{X})=\mathbb{R}^{2}, \\
0, & \text { otherwise, }\end{cases}
\end{aligned}
$$

where $\int M=1$. Recall that $M(\cdot \mid \boldsymbol{X})=M(\cdot \mid \boldsymbol{Y})$ for any permutation $\boldsymbol{Y}$ of the columns of $\boldsymbol{X}$. For $\boldsymbol{x}$ not on any of the knot lines we have the recurrence relation 17.

$$
Q(\boldsymbol{x} \mid \boldsymbol{X})=\beta_{i}^{i, j, k} Q\left(\boldsymbol{x} \mid \boldsymbol{X}_{i}\right)+\beta_{j}^{i, j, k} Q\left(\boldsymbol{x} \mid \boldsymbol{X}_{j}\right)+\beta_{k}^{i, j, k} Q\left(\boldsymbol{x} \mid \boldsymbol{X}_{k}\right),
$$

where $\boldsymbol{X}_{r}:=\boldsymbol{X} \backslash\left\{\boldsymbol{x}_{r}\right\}$ is defined by removing one of the knots equal to $\boldsymbol{x}_{r}$ from $\boldsymbol{X}$ and the $\beta^{i, j, k}$, s are the barycentric coordinates of $\boldsymbol{x}$ with respect to a nondegenerate triangle $\operatorname{ch}\left(\left\{\boldsymbol{p}_{i}, \boldsymbol{p}_{j}, \boldsymbol{p}_{k}\right\}\right)$. In the quadratic case we will also use the knot-insertion 
relation [17]

$$
\begin{aligned}
Q(\boldsymbol{x} \mid \boldsymbol{X})= & \beta_{i}^{i, j, k}(\boldsymbol{y}) Q\left(\boldsymbol{x} \mid \boldsymbol{X}_{i} \cup\{\boldsymbol{y}\}\right)+\beta_{j}^{i, j, k}(\boldsymbol{y}) Q\left(\boldsymbol{x} \mid \boldsymbol{X}_{j} \cup\{\boldsymbol{y}\}\right) \\
& +\beta_{k}^{i, j, k}(\boldsymbol{y}) Q\left(\boldsymbol{x} \mid \boldsymbol{X}_{k} \cup\{\boldsymbol{y}\}\right),
\end{aligned}
$$

where the $\beta^{i, j, k}$ 's are now the barycentric coordinates of $\boldsymbol{y}$ with respect to $\operatorname{ch}\left(\left\{\boldsymbol{p}_{i}, \boldsymbol{p}_{j}\right.\right.$, $\left.\boldsymbol{p}_{k}\right\}$ ) and we add the inserted knot $\boldsymbol{y}$ to the knots on the right in (A.3). We need to express the barycentric coordinates of $\beta_{r}^{i, j, k}$ for $r=i, j, k$ in terms of $\beta_{r}$ for $r=1,2,3$. A few samples that we need are:

$$
\begin{array}{lll}
\beta_{1}^{1,4,6}=\gamma_{1}, & \beta_{4}^{1,4,6}=2 \beta_{2}, & \beta_{6}^{1,4,6}=2 \beta_{3}, \\
\beta_{1}^{1,2,5}=\beta_{1}, & \beta_{2}^{1,2,5}=\beta_{2,3}, & \beta_{5}^{1,2,5}=2 \beta_{3}, \\
\beta_{1}^{1,2,6}=\beta_{1,3}, & \beta_{2}^{1,2,6}=\beta_{2}, & \beta_{6}^{1,2,6}=2 \beta_{3}, \\
\beta_{4}^{4,5,6}=-\gamma_{3}, & \beta_{5}^{4,5,6}=-\gamma_{1}, & \beta_{6}^{4,5,6}=-\gamma_{2}, \\
\beta_{1}^{1,2,10}=\beta_{1,3}, & \beta_{2}^{1,2,10}=\beta_{2,3}, & \beta_{10}^{1,2,10}=3 \beta_{3} .
\end{array}
$$

Using the recurrence relation (A.2), the equations A.4 and the normalization (A.1) we determine column $j$ of $\boldsymbol{R}_{1}$ corresponding to $S_{j, 1}$ for $j=1,4,7,10$. As in Definition 2.1 we set $v_{j, 1}:=v\left(\operatorname{supp}\left(\boldsymbol{K}_{j, 1}\right)\right)$. and apply the shorthand notation

$$
Q\left(i_{1}, \ldots, i_{n}\right):=Q\left(\cdot \mid\left[\boldsymbol{p}_{i_{1}}, \ldots, \boldsymbol{p}_{i_{n}}\right]\right), \quad S\left(i_{1}, \ldots, i_{n}\right):=S\left(\cdot \mid\left[\boldsymbol{p}_{i_{1}}, \ldots, \boldsymbol{p}_{i_{n}}\right]\right) .
$$

Consider first the corner hat $S_{1,1}$.

$$
\begin{aligned}
S_{1,1} & =v_{1,1} Q(1,1,4,6) \\
& =v_{1,1}\left(\beta_{1}^{1,4,6} Q(1,4,6)+\beta_{4}^{1,4,6} Q(1,1,6)+\beta_{6}^{1,4,6} Q(1,1,4)\right) \\
& =v_{1,1} \gamma_{1} \frac{S(1,4,6)}{v_{1,1}}=v_{1,1} \gamma_{1} \frac{S_{1,0}+S_{2,0}}{v_{1,1}}=\gamma_{1} S_{1,0}+\gamma_{1} S_{2,0} .
\end{aligned}
$$

This determines the first column of $\boldsymbol{R}_{1}$ and it is easy to see that $\gamma_{1} S_{i, 0}(\boldsymbol{x}) \geq 0$ for $i=1,2$ and $\boldsymbol{x} \in \Delta$.

For the edge hat $S_{4,1}$ we obtain

$$
\begin{aligned}
S_{4,1} & =v_{4,1} Q(1,4,2,10) \\
& =v_{4,1}\left(\beta_{1}^{1,2,10} Q(4,2,10)+\beta_{2}^{1,2,10} Q(1,4,10)+\beta_{10}^{1,2,10} Q(1,4,2)\right) \\
& =v_{4,1}\left(\beta_{1,3} \frac{S(4,2,10)}{v_{4,1} / 2}+\beta_{2,3} \frac{S(1,4,10)}{v_{4,1} / 2}\right) \\
& =v_{4,1}\left(\beta_{1,3} \frac{S_{3,0}+S_{9,0}}{v_{4,1} / 2}+\beta_{2,3} \frac{S_{2,0}+S_{8,0}}{v_{4,1} / 2}\right) \\
& =2\left(\beta_{2,3} S_{2,0}+\beta_{1,3} S_{3,0}+\beta_{2,3} S_{8,0}+\beta_{1,3} S_{9,0}\right),
\end{aligned}
$$

giving the 4th column of $\boldsymbol{R}_{1}$. Moreover, $\beta_{2,3} S_{i, 0}(\boldsymbol{x}) \geq 0$ and $\beta_{1,3} S_{k, 0}(\boldsymbol{x}) \geq 0$ for $i=2,8, k=3,9$ and $\boldsymbol{x} \in \Delta$. Consider next the 7 th column of $\boldsymbol{R}_{1}$ corresponding to the Egyptian pyramid $S_{7,1}$

$$
\begin{aligned}
S_{7,1} & =v_{7,1} Q(1,4,10,6) \\
& =v_{7,1}\left(\beta_{1}^{1,4,6} Q(4,10,6)+\beta_{4}^{1,4,6} Q(1,10,6)+\beta_{6}^{1,4,6} Q(1,4,10)\right) \\
& =v_{7,1}\left(\gamma_{1} \frac{S_{7,0}+S_{8,0}}{v_{7,1} / 4}+2 \beta_{2} \frac{S_{1,0}+S_{7,0}}{v_{7,1} / 2}+2 \beta_{3} \frac{S_{2,0}+S_{8,0}}{v_{7,1} / 2}\right) \\
& =4\left(\beta_{2} S_{1,0}+\beta_{3} S_{2,0}+\beta_{1,3} S_{7,0}+\beta_{1,2} S_{8,0}\right),
\end{aligned}
$$


and again all terms on the right are nonnegative on $\Delta$. Finally, for the center pyramid we obtain

$$
\begin{aligned}
S_{10,1} & =v_{10,1} Q(4,5,6,10) \\
& =v_{10,1}\left(\beta_{4}^{4,5,6} Q(5,6,10)+\beta_{5}^{4,5,6} Q(6,4,10)+\beta_{6}^{4,5,6} Q(4,5,10)\right) \\
& =v_{10,1}\left(-\gamma_{3} \frac{S_{11,0}+S_{12,0}}{v_{10,1} / 3}-\gamma_{1} \frac{S_{7,0}+S_{8,0}}{v_{10,1} / 3}-\gamma_{2} \frac{S_{9,0}+S_{10,0}}{v_{10,1} / 3}\right) \\
& =-3\left(\gamma_{1}\left(S_{7,0}+S_{8,0}\right)+\gamma_{2}\left(S_{9,0}+S_{10,0}\right)+\gamma_{3}\left(S_{11,0}+S_{12,0}\right)\right),
\end{aligned}
$$

which determines column 10 in $\boldsymbol{R}_{1}$. Also the nonnegativity assertion follows. The remaining columns follow by symmetry.

In the quadratic case the recursions are shown for the first columns of $\boldsymbol{R}_{2}$ corresponding to the typical cases $S_{j, 2}, j=1,2,3$. We let $v$ be the area of the triangle $\Delta$. For the corner S-spline, the relation

$$
S_{1,2}=\frac{v}{4} Q(1,1,1,4,6) \stackrel{\text { A.2 }}{=} \frac{v}{4} \beta_{1}^{1,4,6} Q(1,1,4,6)=\frac{v}{4} \gamma_{1} \frac{4}{v} S_{1,1}=\gamma_{1} S_{1,1},
$$

gives the first column of $\boldsymbol{R}_{2}$, and nonnegativity follows. For the second column we need to show that

$$
S_{2,2}=2 \beta_{2} S_{1,1}+\beta_{1,3} S_{4,1}+\frac{1}{2} \beta_{1,3} S_{7,1} .
$$

Clearly, all terms on the right are nonnegative on $\Delta$. Now

$$
S_{2,2}=\frac{v}{2} Q(1,1,4,2,6) \stackrel{\text { A.2 }}{=} \frac{v}{2} \beta_{1}^{1,2,6} Q(1,4,2,6)+\frac{v}{2} \beta_{2}^{1,2,6} Q(1,1,4,6)
$$

The first term does not have the knots of any of the $S_{j, 1}$. We correct this by inserting the knot $\boldsymbol{p}_{10}$ according to

$$
\begin{aligned}
\frac{v}{2} \beta_{1}^{1,2,6} Q(1,4,2,6) & \stackrel{\sqrt{\mathrm{A} .3})}{=} \frac{v}{2} \beta_{1,3}\left(\beta_{1}^{1,2,6}\left(\boldsymbol{p}_{10}\right) Q(4,2,6,10)\right. \\
& \left.+\beta_{2}^{1,2,6}\left(\boldsymbol{p}_{10}\right) Q(1,4,6,10)+\beta_{6}^{1,2,6}\left(\boldsymbol{p}_{10}\right) Q(1,4,2,10)\right) \\
& =\frac{v}{2} \beta_{1,3}\left(\frac{1}{3} Q(1,4,10,6)+\frac{2}{3} Q(1,4,2,10)\right) \\
& =\frac{v}{6} \beta_{1,3}\left(\frac{3}{v} S_{7,1}+2 \frac{3}{v} S_{4,1}\right)=\beta_{1,3} S_{4,1}+\frac{1}{2} \beta_{1,3} S_{7,1} .
\end{aligned}
$$

Adding the second term $\frac{v}{2} \beta_{2}^{1,2,6} Q(1,1,4,6)=\frac{v}{2} \beta_{2} \frac{4}{v} S_{1,1}=2 \beta_{2} S_{1,1}$ we obtain A.10. Finally, we derive the recurrence relation

$$
S_{3,2}=3 \beta_{3} S_{4,1}+\frac{3}{2} \beta_{2} S_{7,1}+\frac{3}{2} \beta_{1} S_{8,1}+\left(-\gamma_{3}\right) S_{10,1}
$$

To start

$$
\begin{aligned}
S_{3,2} & =\frac{3 v}{4} Q(1,4,2,5,6) \\
& \stackrel{\text { A.2 }}{=} \frac{3 v}{4}\left(\beta_{1}^{1,2,6} Q(4,2,5,6)+\beta_{2}^{1,2,6} Q(1,4,5,6)+\beta_{6}^{1,2,6} Q(1,4,2,5)\right) .
\end{aligned}
$$

None of the knot sets of these linear simplex splines is among the $S_{j, 1}$, so we need to use knot insertion for all 3 terms on the right of the equality sign. For the first 
term,

$$
\begin{aligned}
\frac{3 v}{4} \beta_{1}^{1,2,6} Q(4,2,5,6) & =\frac{3 v}{4} \beta_{1,3}\left(\beta_{2}^{2,5,6}\left(\boldsymbol{p}_{10}\right) Q(4,5,6,10)\right. \\
& \left.+\beta_{5}^{2,5,6}\left(\boldsymbol{p}_{10}\right) Q(4,2,6,10)+\beta_{6}^{2,5,6}\left(\boldsymbol{p}_{10}\right) Q(4,2,5,10)\right) \\
& =\frac{3 v}{4} \beta_{1,3}\left(\frac{1}{3} \frac{4}{v} S_{10,1}+\frac{2}{3} \frac{3}{v} S_{8,1}\right)=\beta_{1,3}\left(\frac{3}{2} S_{8,1}+S_{10,1}\right),
\end{aligned}
$$

and similarly for the second and third term,

$$
\begin{aligned}
& \frac{3 v}{4} \beta_{2}^{1,2,6} Q(1,4,5,6)=\beta_{2}\left(\frac{3}{2} S_{7,1}+S_{10,1}\right), \\
& \frac{3 v}{4} \beta_{6}^{1,2,6} Q(1,4,2,5)=\beta_{3}\left(3 S_{4,1}+\frac{3}{2} S_{8,1}\right) .
\end{aligned}
$$

This proves (A.11). Again each of the 4 terms on the right of (A.11) are nonnegative on $\Delta$. This completes the proof.

\section{ACKNOWLEDGEMENTS}

The authors would like to thank the Centre of Mathematics for Applications (CMA) at the University of Oslo for support and encouragement of this work.

\section{REFERENCES}

1. P. Alfeld, and L. Schumaker, The dimension of bivariate spline spaces of smoothness $r$ for degree $d \geq 4 r+1$, Constr. Approx. 3 (1987), 891-911. MR889554 (88e:41025)

2. C. de Boor, K. Höllig, and S. Riemenschneider, Box Splines, Springer-Verlag, New York, 1993. MR1243635 (94k:65004)

3. S. C. Brenner and L. R. Scott, The Mathematical Theory of Finite Element Methods, 2nd ed., Springer-Verlag, New York, 2002. MR.1894376 (2003a:65103)

4. E. Cohen, T. Lyche, and R. F. Riesenfeld, Discrete B-splines and subdivision techniques in computer aided geometric design and computer graphics, Computer Graphics and Image Processing 14 (1980), 87-111.

5. W. Dahmen, On multivariate B-splines, SIAM J. Numer. Anal. 17 (1980), 179-191. MR567267 (81c:41020)

6. W. Dahmen and C. A. Micchelli, On the linear independence of multivariate B-splines I, Triangulations of simploids, SIAM J. Numer. Anal. 19 (1982), 993-1012. MR672573(85c:41016a)

7. W. Dahmen, C. A. Micchelli, and H-P Seidel, Blossoming begets B-splines built better by B-patches, Math. Comp 59 (1992), 97-115. MR.1134724 (93b:41014)

8. P. Dierckx, On calculating normalized Powell-Sabin B-splines, Comput. Aided Geom. Design, 15 (1997), 61-78. MR1484258 (98j:41004)

9. N. Dyn and T. Lyche, A Hermite subdivision scheme for the evaluation of the Powell-Sabin 12-split element, in Approximation Theory IX, Volume 2, Charles Chui and Larry Schumaker (eds.), Vanderbilt University Press, Nashville, 1998, 33-38. MR.1743030

10. T. A. Grandine, The computational cost of simplex spline functions, SIAM J. Numer. Anal. 24 (1987), 887-890. MR 899710 (88j:41032)

11. T. A. Grandine, The stable evaluation of multivariate simplex splines, Math. Comp. 50 (1988), 197-205. MR917827 (89a:65018)

12. K. Höllig, Multivariate splines, SIAM J. Numer. Anal. 19 (1982), 1013-1031. MR672574 (84i:41013)

13. Ming-Jun Lai and Larry L. Schumaker, Spline Functions on Triangulations, Cambridge University Press, Cambridge, 2007. MR2355272 (2008i:41001)

14. J. M. Lane and R. F. Riesenfeld, A theoretical development for the computer generation and display of piecewise polynomial surfaces, IEEE Trans. Pattern Anal. Mach. Intellig 2 (1980), $35-46$.

15. T. Lyche and K. Scherer, On the p-norm condition number of the multivariate triangular Bernstein basis, J. Comput. Appl. Math. 119 (2000), 259-273. MR1774222 (2001h:41009) 
16. J. Maes, E. Vanraes, P. Dierckx, and A. Bultheel, On the stability of normalized Powell-Sabin B-splines, J. Comput. Appl. Math. 170 (2004), 181-196. MR2075830 (2005f:41029)

17. C.A. Micchelli, On a numerically efficient method for computing multivariate B-splines, in Multivariate Approximation Theory, (W. Schempp and K. Zeller, eds.), Birkhäuser Verlag, Basel, 1979, 211-248. MR560673 (81g:65017)

18. M. Neamtu, What is the natural generalization of univariate splines to higher dimensions?, in Mathematical Methods in CAGD: Oslo 2000, Tom Lyche and Larry L. Schumaker (eds.), Vanderbilt University Press, Nashville, 2001, 355-392. MR.1858970

19. M. Neamtu, Delaunay configurations and multivariate splines: A generalization of a result of B. N. Delaunay, Trans. Amer. Math. Soc. 359 (2007), 2993-3004. MR2299443(2008c:65042)

20. M. J. D. Powell and M. A. Sabin, Piecewise quadratic approximation on triangles, ACM Trans. Math. Software 3 (1977), 316-325. MR0483304 (58:3319)

21. L. L. Schumaker and T. Sorokina, Smooth macro-elements on Powell-Sabin-12 splits, Math. Comp. 75 (2006), 711-726. MR2196988 (2006m:65284)

22. H-P Seidel, Polar forms and triangular B-spline surfaces, in Blossoming: The New Polar Form Approach to Spline Curves and Surfaces, Siggraph'91 Course Notes \#26, World Scientific Publishing Company, 1992, 235-286. MR1239195

23. H. Speleers, P. Dierckx, and S. Vandewalle, On the Lp-stability of quasi-hierarchical PowellSabin B-splines, In Approximation Theory XII: San Antonio 2007, M. Neamtu and L.L. Schumaker (eds.), Nashboro Press, 2008, 398-413. MR2544025 (2010i:65025)

24. H. Speleers, P. Dierckx, and S. Vandewalle, On the local approximation power of quasihierarchical Powell-Sabin splines, In Mathematical Methods for Curves and Surfaces, M. Dæhlen, M. S. Floater, T. Lyche, J-L. Merrien, K. Mørken and L. L. Schumaker (eds.), Lecture Notes in Computer Science LNCS 5862, 2010, 419-433.

25. H. Speleers, A normalized basis for quintic Powell-Sabin splines, Comput. Aided Geom. Design, 27 (2010), 438-457. MR2657545 (2011j:65034)

School of Computing, University of Utah, Salt Lake City, Utah 84112

E-mail address: cohen@cs.utah.edu

CMA and Institute of Informatics, University of Oslo, PO Box 1053, Blindern, 0316 OSLO, NORWAY

E-mail address: tom@ifi.uio.no

School of Computing, University of Utah, Salt Lake City, Utah 84112

E-mail address: rfr@cs.utah.edu 